\title{
Palladium(II)-Catalysed Oxidation of Alkenes
}

\author{
Sam E. Mann, ${ }^{\mathrm{a}}$ L. Benhamou, ${ }^{\mathrm{b}}$ Tom D. Sheppard ${ }^{\mathrm{b}}$ \\ ${ }^{a}$ Argenta, Discovery Services, Charles River, 7-9 Spire Green Centre, Flex Meadow, Harlow, Essex, CM19 5TR, U.K. \\ ${ }^{\mathrm{b}}$ Department of Chemistry, University College London, Christopher Ingold Laboratories, 20 Gordon St, London, WC1H 0AJ, U.K. \\ E-mail: tom.sheppard@ucl.ac.uk. \\ Received: The date will be inserted once the manuscript is accepted. \\ Dedication - If you wish to insert a short dedication please overwrite this text, otherwise delete the paragraph.
}

\begin{abstract}
This review provides a summary of recent developments in the $\mathrm{Pd}(\mathrm{II})$-catalysed oxidation of alkenes, focusing largely on reactions which lead to the formation of new carbon-oxygen or carbon-nitrogen bonds. Three classes of reaction are covered: i) Oxidation proceeding via allylic $\mathrm{C}-\mathrm{H}$ bond cleavage and formation of a $\pi$-allyl complex; ii) Wackertype oxidations proceeding via nucleopalladation followed by $\beta$-hydride eliminations; and iii) 1,2-Difunctionalisation of alkenes proceeding via nucleopalladation followed by functionalisation of the resulting $\sigma$-alkylpalladium(II) intermediate. The mechanisms are discussed alongside the scope and limitations of each reaction.
\end{abstract}

1 Introduction

$1.1 \quad$ Background

1.2 Oxidation Pathways

1.3 Observation of Reaction Intermediates

2 Allylic Oxidation

2.1 Background

2.2 Allylic Oxygenation

2.3 Allylic Amination

2.4 Allylic Functionalisation with other Nucleophiles

The Wacker oxidation

Background

Variation of the Co-Oxidant

Direct Oxygen-Coupled Wacker Oxidations

Aldehyde-Selective Wacker Oxidations

Wacker Oxidation of Internal Alkenes

Aza-Wacker Oxidations

Intermolecular 1,2-Difunctionalisation of Alkenes

Introduction

Oxyhalogenation Reactions

Dioxygenation Reactions

Oxycarbonylation Reactions

Aminohalogenation Reactions

Diamination Reactions

Aminooxygenation Reactions

Aminocarbonylation Reactions

Summary and Conclusions

Key words: Alkenes, Oxidation, Palladium, Wacker oxidation, Catalysis

\section{Introduction}

\subsection{Background}

Alkenes are extremely abundant chemical feedstocks which are produced in large quantities from petrochemical sources. As a consequence, they have been exploited as starting materials for the synthesis of a wide range of organic chemical building blocks. The oxidation of alkenes to introduce carbon-oxygen and other carbon-heteroatom bonds is a highly important process which enables higher polarity molecules to be prepared from these abundant hydrocarbons. Metal-catalysed oxidation reactions are particularly notable in this respect and a palladium- catalysed alkene oxidation - The Wacker Oxidation was one of the first transition-metal catalysed industrial processes, enabling the efficient generation of acetaldehyde from ethylene. ${ }^{1}$

Since this pioneering process was developed, the field has grown considerably and a range of palladiumcatalysed oxidation reactions are routinely used in synthetic chemistry laboratories all over the world. These reactions generally rely on the strong interaction of palladium(II) salts with the $\pi$-orbitals of an alkene, which opens up several different reaction pathways via which new carbon-heteroatom bonds can be formed (Scheme 1). Importantly, these pathways are often finely balanced, with similar substrates sometimes undergoing oxidation via different mechanisms. The choice of catalyst and/or ligand can provide high levels of control over the reaction pathway though, enabling different products to be accessed from the same substrate. This review will cover the diverse methods available for the palladium-catalysed oxidation of alkenes, discussing the likely mechanisms of the reactions in each case and outlining potential new directions for research in this area.

\subsection{Oxidation Pathways}

Coordination of an alkene $\mathbf{1}$ (Scheme 1) to a palladium(II) salt renders a typically electron rich alkene considerably more electrophilic 2, allowing it to be attacked by oxygen nucleophiles such as water, alcohols, or acetate ions. One common pathway involves initial abstraction of an allylic proton by the nucleophile (or a base) to generate $\pi$-allyl complex $\mathbf{3}$ (Path 1). This relatively stable complex can then undergo a nucleophilic substitution reaction, generally via an outer sphere process, to give allylic oxidation products $\mathbf{4}$ and $\mathbf{5}$, together with a palladium $(0)$ species, which must be re-oxidised to palladium(II) in order for the catalytic cycle to be completed. Alternative reaction pathways involve direct attack of the nucleophile onto the activated alkene 2 to give a $\sigma$-alkylpalladium intermediate $\mathbf{6 a}$ (and/or its regioisomer $\mathbf{6 b}$ ) via a concerted nucleopalladation. ${ }^{2}$ Intermediate $\mathbf{6 a}$ can then undergo $\beta$-hydride elimination (Path 2) to give either vinylic oxidation product $\mathbf{7}$, or allylic oxidation product $\mathbf{4}$ depending on the regioselectivity of the $\beta$-hydride elimination. In the Wacker oxidation, the nucleophile is typically water and the resulting enol $7(\mathrm{Nu}=\mathrm{OH})$ undergoes tautomerisation to the ketone or aldehyde. It should be 
noted, however, that even this tautomerisation is thought to be mediated by palladium. ${ }^{3}$ In combination with the formation of regioisomer $\mathbf{6 b}$ in the initial nucleopalladation process, pathway 2 can lead to the formation of up to four different possible oxidation products. $\beta$-Hydride elimination generates an $\mathrm{HPdX}$ species which can readily undergo reductive elimination to extrude $\mathrm{HX}$, and re-oxidation to an active $\mathrm{Pd}(\mathrm{II})$ species is again necessary in order to complete the cycle.

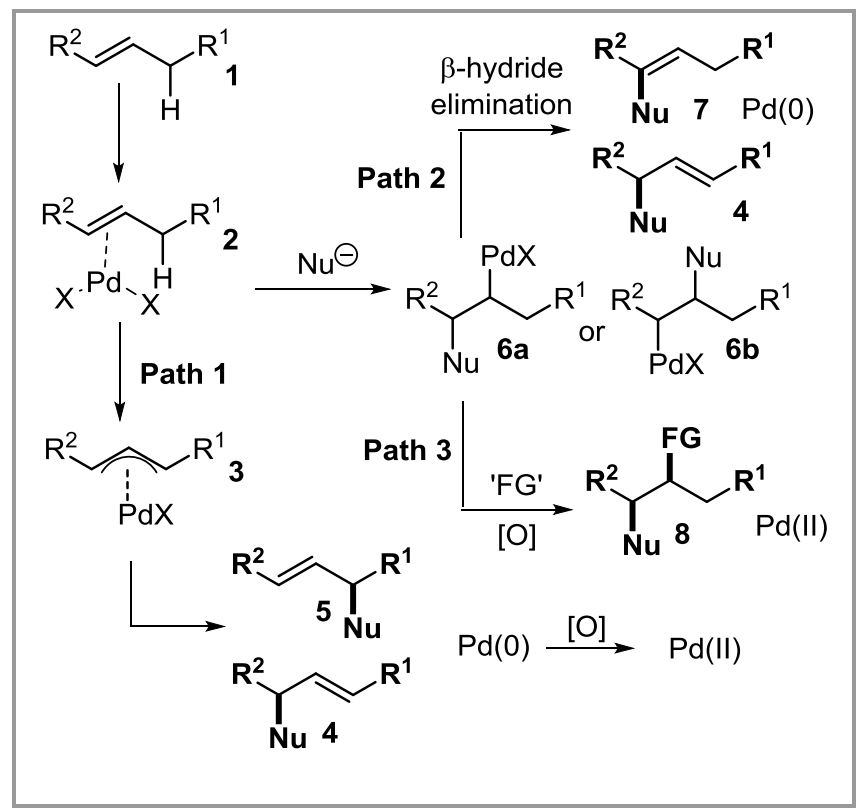

Scheme 1 Pathways for palladium-catalysed alkene oxidation

A third oxidation pathway involves further functionalisation of the $\sigma$-palladium intermediates $\mathbf{6 a -}$ 6b with an oxidizing agent or trapping reagent to generate a new $\sigma$-bond at the carbon where the palladium is bound (Path 3), giving a 1,2difunctionalisation product 8. Often, this final pathway leads to the regeneration of a $\mathrm{Pd}(\mathrm{II})$ species directly so no further oxidation of the catalyst is required. Again, a number of isomeric products can be obtained depending on both the regioselectivity and stereoselectivity of the initial nucleopalladation reaction and the mechanism of the subsequent oxidative functionalisation (retention or inversion of the stereochemistry of the carbon-palladium bond).

From the three different pathways discussed above and depicted in Scheme 1, it can be seen that the mechanism of a particular reaction can often be far from obvious. For example, the product of a formal allylic oxidation with alkene transposition 4 can be obtained from two different reaction pathways (Path 1 and Path 2), and the situation can be complicated further by the fact that alkenes in both the products and the starting materials can potentially undergo isomerisation under the reaction conditions.

This review will cover recent developments in the Pd(II) catalysed oxidation of alkenes via the three pathways shown in Scheme 1, with a focus on intermolecular reactions that involve the formation of a new carbon-oxygen or carbon-nitrogen bond.

\subsection{Observation of Reaction Intermediates}

The direct observation of the intermediates 2, 3 and $\mathbf{6}$ is possible in many cases, and it is clear that the different reaction pathways by which a particular alkene undergoes oxidation are often finely balanced. Notably, the incorporation of ligands for palladium into the substrate can lead to stabilization of both $\pi$ allyl palladium complexes and $\sigma$-palladium complexes. $\pi$-Allyl palladium complexes can also be isolated in the absence of a suitable trapping nucleophile. ${ }^{4}$ In our own work, we have studied the Pd-catalysed oxidation of unsaturated thioacetals, ${ }^{5}$ which provides a useful framework for direct observation of the various organopalladium intermediates involved in alkene oxidation. This work also gives a good illustration of the fine balance between the different reaction pathways. Oxidation of closely related substrates $\mathbf{9}$ and $\mathbf{1 0}$ using a palladium(II) catalyst in the presence of $p$ benzoquinone (BQ) and $\mathrm{MnO}_{2}$ as co-oxidants led to the formation of reaction products which apparently derived from different mechanistic pathways (Scheme 2). Product 11 appears to be the result of either a linear-selective allylic oxidation (Path 1) or a Wacker type oxypalladation (Path 2 ) with regioselective $\beta$ hydride elimination away from the acetate. In contrast, product 12, derived from sterically crowded substrate 10, appears to result from a Wacker-type oxypalladation reaction followed by regioselective $\beta$ hydride elimination towards the acetate group (Path 2 ). The oxypalladation process seems to occur with opposite regioselectivity to a typical Wacker oxidation (which usually gives methyl ketones from terminal alkenes), presumably due to the presence of the sulfur ligand in the substrate which directs the palladium towards the more hindered internal carbon atom. In the case of other substrates, mixtures of the two classes of products were obtained and these were demonstrated not to isomerise under the reaction conditions.

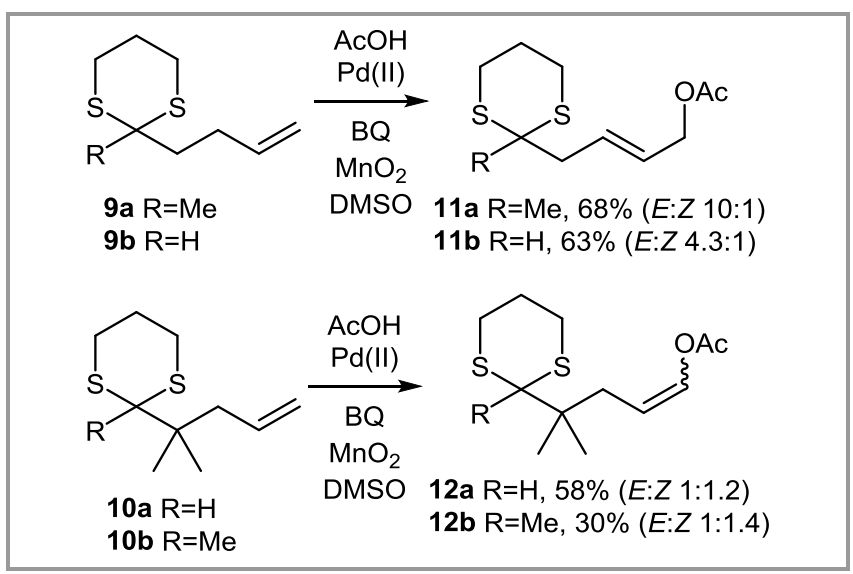

Scheme 2 Formation of different products from the palladium(II)mediated oxidation of closely related unsaturated thioacetals. 
The dithiane group is a good bidentate ligand for palladium(II) which readily coordinates to $\mathrm{Pd}(\mathrm{OAc})_{2}$. This leads to the formation of a well-defined complex in which there is a remarkable downfield shift of the nearby axial proton on the dithiane ring (Scheme 3). In computational calculations, this effect was attributed to the close proximity of this proton to the acetate ligands on palladium. Reaction of thioacetal 9a with $\mathrm{Pd}(\mathrm{OAc})_{2}$ led first to the formation of an isomeric mixture of complexes 13a-13b, which gradually converted into a single $\pi$-allyl complex $\mathbf{1 4}$. This in turn was gradually converted into the allylic oxidation product 11a, showing that this compound is formed exclusively via Path 1 . With substrate 10a, initial formation of the thioacetal complex 15 was stereoselective, but the probable $\sigma$-organopalladium intermediate $\mathbf{1 6}$ was not observed, with direct formation of the oxidation product 12a being seen by NMR. However, it should be noted that a Wacker-type oxidation (Path 2) is the most plausible mechanism for the formation of this compound. It was however, possible to obtain unusually stable $\sigma$-alkylpalladium complexes 18a-18b by reaction of truncated substrates 17a-17b with $\mathrm{Pd}(\mathrm{OAc})_{2}$. Interestingly, in complexes 18a-18b the new carbon-palladium bond was formed with complete diastereoselectivity. These compounds are remarkably resistant to $\beta$-hydride elimination, but can undergo further reactions. The carbon-palladium bond can be functionalised with concomitant dithiane removal by treatment with a strong oxidizing agent, providing a step-wise demonstration of a 1,2-alkene difunctionalisation reaction (Path 3).

A potential reason for the different reactivity observed with these substrates is the conformation of the sixmembered dithiane ring, and the orientation of the alkene-containing chain. In the case of $\mathbf{1 0 a}-\mathbf{1 0 b}$, the bulky gem-dimethyl group forces the alkenecontaining chain to occupy an equatorial position on the dithiane ring, which appears to promote direct oxypalladation. However, an axial alkene group (complex 13b) appears to promote $\pi$-allyl formation (14) which is able to out-compete direct oxypalladation of 13a in a Curtin-Hammett type equilibrium for substrate 9 a.

The above observations serve to illustrate the fine balance between the competing oxidation pathways: Reaction of a simple alkene with $\mathrm{Pd}(\mathrm{OAc})_{2}$ can proceed either via direct formation of a $\pi$-allyl complex or through oxypalladation of the alkene, depending on the fine structure of the substrate. As will be seen in the discussion that follows, this can complicate the development of new reaction manifolds as subtle factors can affect which pathway is followed.

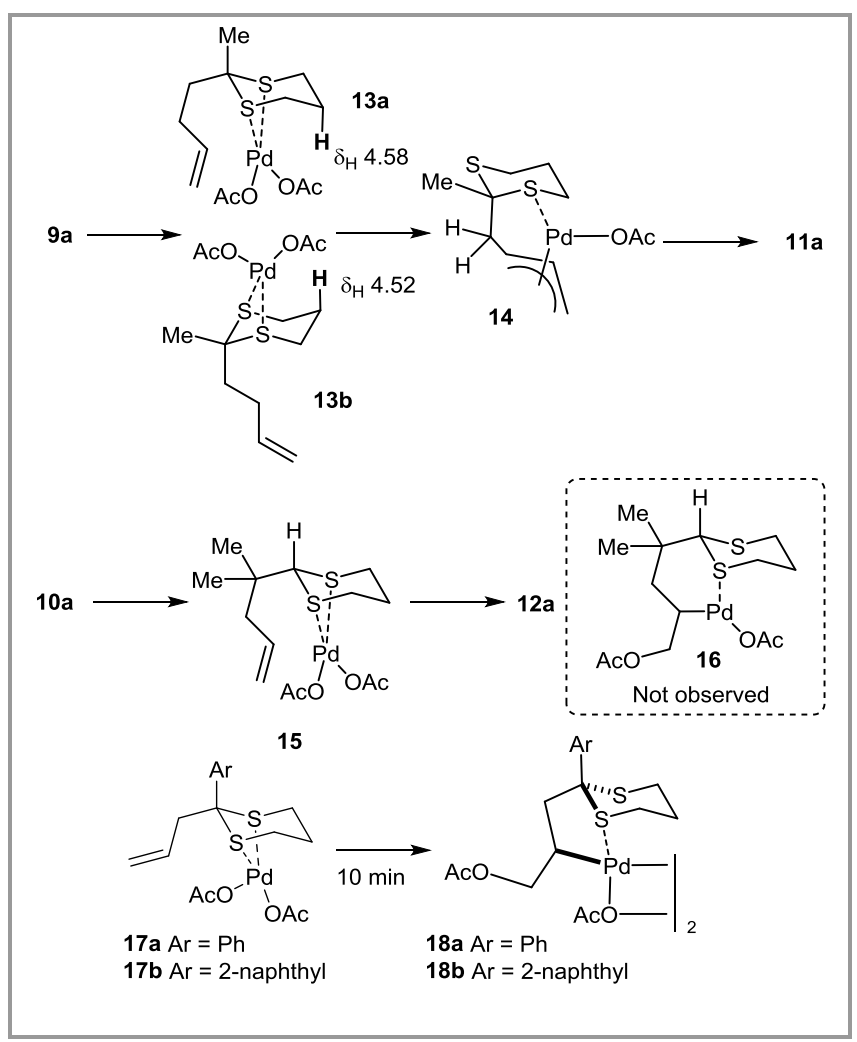

Scheme 3 Observation of divergent reaction mechanisms in the palladium(II)-mediated oxidation of closely-related unsaturated thioacetals.

\section{Allylic Oxidation}

With an abundance of alkenes available as starting materials, methodologies that enable the synthetic chemist to transform simple hydrocarbons into more complex heteroatom-containing compounds are highly desirable. Oxidation at the activated allylic position is one approach that has been heavily exploited and provides a reliable method for incorporating heteroatoms into aliphatic building blocks. The resultant allylic alcohols and amines have a variety of applications in synthesis, ranging from simple enone precursors to complex substrates for the Tsuji-Trost reaction ${ }^{6}$ and Sharpless asymmetric epoxidation. ${ }^{7}$ As well as being useful synthetic intermediates, allylic alcohols and amines are common structural features found in a number of biologically active natural products and medicines, making them attractive targets in their own right. There are many methods available for the allylic oxidation of alkenes, ${ }^{8}$ including the use of stoichiometric quantities of toxic chromium(VI) reagents ${ }^{8 \mathrm{a}, \mathrm{d}}$ or malodourous organoselenium compounds, ${ }^{8 \mathrm{~d}, \mathrm{e}}$ causing environmental and safety concerns. The KharaschSosnovsky reaction, ${ }^{8 f, g}$ which offers a non-toxic copper-mediated alternative has also been widely used to achieve this useful transformation. ${ }^{8 \mathrm{~b}, \mathrm{c}}$ Whilst reliable, these traditional approaches tend to be incompatible with many functional groups, causing undesired oxidation of alcohols, at benzylic positions and adjacent to heteroatoms and carbonyl groups. The use of suitable protecting groups is often required as a 
result, which involves additional steps and increases the amount of waste generated in the synthesis.

The use of palladium(II) salts for the allylic oxidation of alkenes is a comparatively new process that provides a much milder, more general approach to traditional methods and is compatible with a wider range of functional groups. Although it was first documented as early as the 1960s, the power of this transformation was not fully realized until relatively recently. As a result, the field has received much attention in recent years. However, to the best of our knowledge, the palladium-mediated allylic oxidation reaction has yet to be reviewed in any detail. This article will therefore cover some of the key early breakthroughs as a prelude to the contemporary research in this field.

\subsection{Background}

The palladium-mediated allylic oxidation of alkenes dates back to the 1960s (Scheme 4), when Mosieev first observed the formation of trace amounts of allyl acetate in the reaction between propene and stoichiometric palladium chloride in the presence of $\mathrm{NaOAc} / \mathrm{AcOH}$ (a). ${ }^{9}$ At around the same time, Winstein demonstrated that cyclic alkenes were converted largely to the corresponding allylic esters (b) and higher terminal alkenes gave a larger proportion of the corresponding allylic acetates than propene, using palladium acetate as oxidant (c). ${ }^{10}$ Internal linear alkenes however, gave almost exclusive conversion to branched allylic esters (d), constituting the first examples of selective allylic oxidation with palladium.

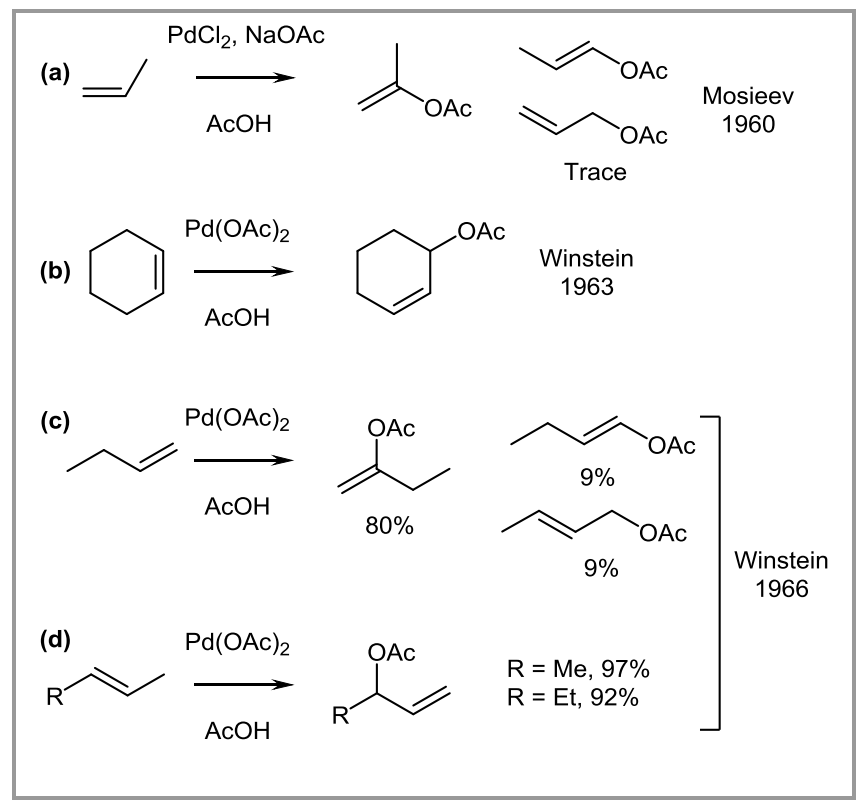

Scheme 4 Early examples of palladium-mediated allylic oxidation of simple alkenes.

Whilst at the time it was not clear what the mechanisms for these particular transformations were, the observation of allyl acetates hinted at the possibility that there was more than one pathway involved in the palladium-mediated oxidation of alkenes. As well as the known Wacker-type 1,2nucleopalladation (Path 2, Scheme 1), the observed allyl acetates could also feasibly have formed via nucleophilic attack by acetate on a $\pi$-allyl palladium complex (Path 1, Scheme 1). As mentioned above, the precise mechanism of these transformations is often unclear, as the same alkene can sometimes undergo oxidation via more than one different pathway and alkene isomerisation can also take place.

Despite these early advances, it was not until much later that a synthetically useful catalytic version of this reaction was developed. Heumann and Åkermark first reported the catalytic allylic oxidation of cyclohexene using an intricate three-component oxidation system comprising palladium acetate, $p$-benzoquinone (BQ) and $\mathrm{MnO}_{2}$ (Scheme 5). ${ }^{11} \mathrm{~A}$ range of simple cyclic and internal linear alkenes were also efficiently oxidized to their corresponding allyl acetates under these conditions.

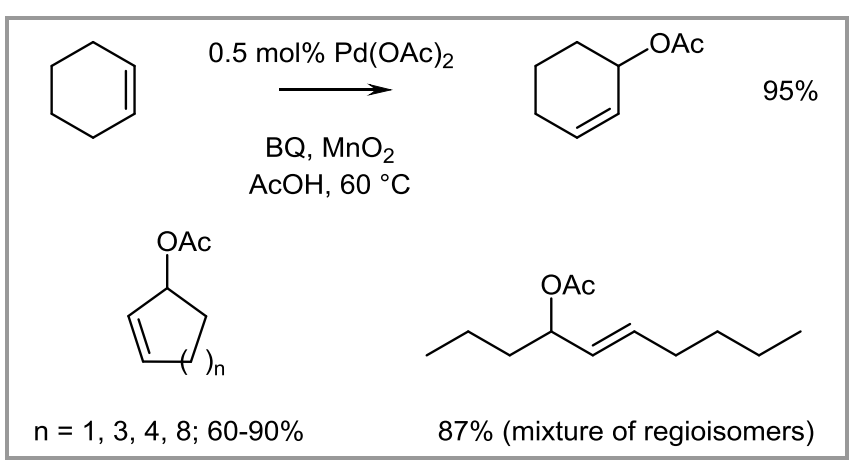

Scheme 5 First example of the catalytic allylic oxidation of alkenes using a palladium(II) salt.

A number of reports followed this breakthrough, extending the scope of the reaction to include alternative co-oxidant systems, different palladium(II) sources and ever more complex substrates. ${ }^{12}$ The palladium(II)-mediated oxidation of alkenes could also be extended to the preparation of higher allyl carboxylates, as demonstrated by Åkermark and coworkers. ${ }^{12 \mathrm{~d}}$ Replacement of acetic acid in the reaction mixture with two equivalents of a higher carboxylic acid gave rise to the corresponding cyclohexene allyl esters in good yields (Scheme 6). Benzoates, cinnamates and even a bulky pivalate could be obtained under these conditions. One example of a chiral carboxylate was also successfully employed in this reaction, although the diastereoselectivity was very modest. Nevertheless, this opened up the possibility for asymmetric induction in future developments. This reaction was also able to employ tert-butyl hydroperoxide as a low-cost stoichiometric oxidant. 


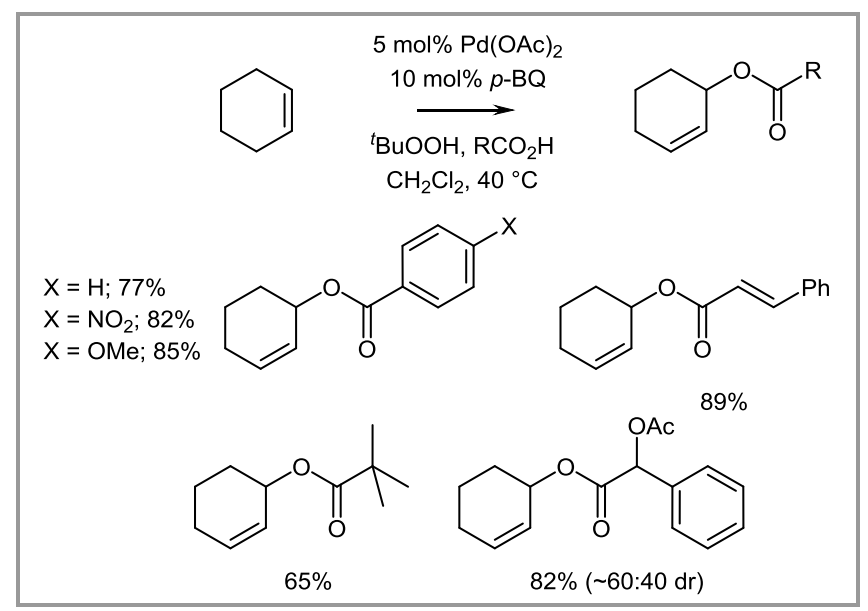

Scheme 6 Formation of higher allyl esters in the palladiummediated oxidation of cyclohexene.

Larock took this idea one step further and developed an elegant intramolecular oxidative allylic cyclisation of alkenoic acids that gave rise to a range of aliphatic lactones (Scheme 7) using molecular oxygen as the stoichiometric oxidant. ${ }^{12 \mathrm{e}}$ Both 5- and 6-membered mono-, bi- and even spirocyclic lactones were formed in excellent yields by this procedure. These species had previously only been accessible via two-step halolactonisation-dehydrohalogenation processes, which clearly demonstrates the advantages of developing this chemistry.

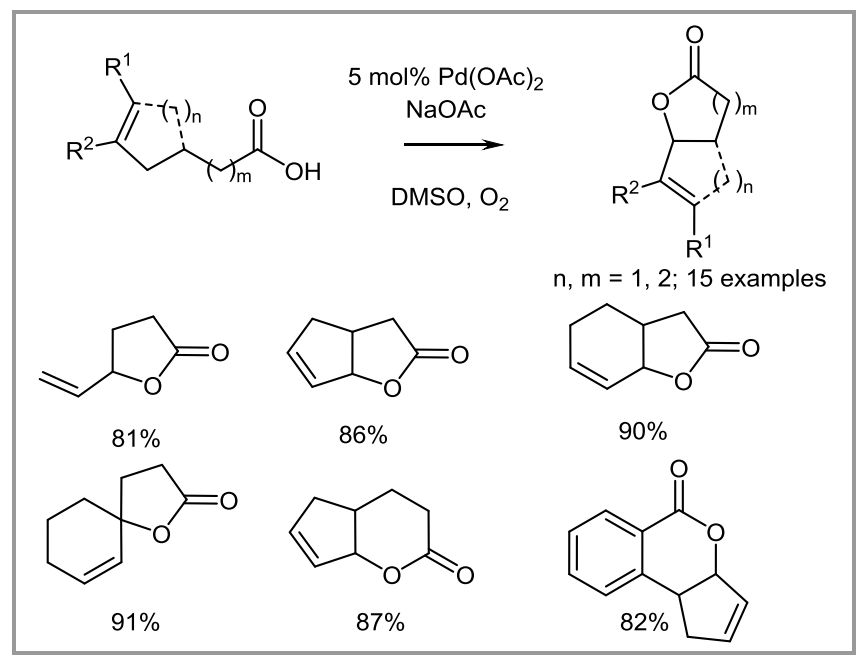

Scheme 7 Intramolecular allylic oxidation to form lactones.

However, some substrates still behaved unpredictably, giving rise to a variety of different oxidized products and a general procedure for allylic oxidation remained elusive. For instance, Åkermark obtained several different products in the palladium acetate/hydrogen peroxide mediated oxidation of a range of alkenes, depending on the nature of the starting material (Scheme 8). ${ }^{12 \mathrm{~d}}$ As demonstrated previously, internal linear and cyclic alkenes gave rise to the expected allyl acetates in good yield (a). Even a complex tetracyclic cholesterol derivative underwent the desired transformation, albeit in much lower yield than the simpler alkenes (b). Oxidation of 1,3cyclohexadiene gave cyclohexene-1,4-diacetate, which has been postulated to proceed via 1,2acetoxypalladation to give a $\pi$-allyl intermediate, followed by subsequent reaction with acetate $(\mathbf{c}) .^{13}$ Terminal alkenes on the other hand underwent exclusive 1,2-oxypalladation to give Wacker products (d). With cis-1,2-divinylcyclohexane (a 1,5-diene) the reaction proceeded via 1,2-acetoxypalladation as with other terminal alkenes, although in this case the Pd-C $\sigma$-intermediate then underwent an intramolecular Heck reaction to give a bicyclic compound (e).

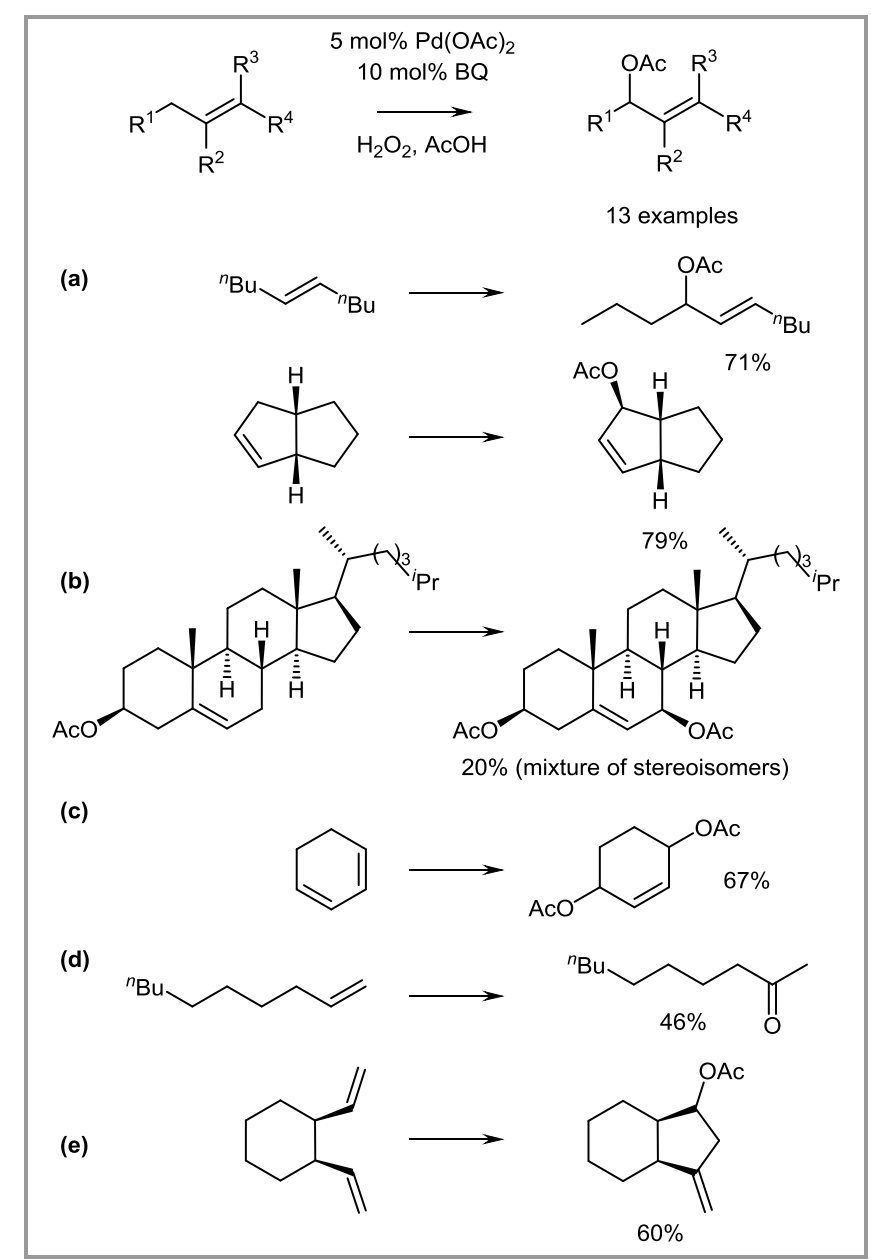

Scheme 8 Different products obtained in the palladium-mediated oxidation of a variety of alkene substrates.

Whilst the diversity of different products available from this chemistry was intriguing, the lack of a more general methodology for palladium-mediated allylic oxidation still represented a significant challenge. In order for the potential of this reaction to be fully realized, a generic procedure that could predictably furnish allyl acetates from alkenes was necessary. This breakthrough came in the form of seminal work by the White group, who developed a sulfoxide-promoted method for the regioselective synthesis of allyl acetates from monosubstituted alkenes. ${ }^{14}$ Depending on the conditions employed, terminal alkenes could be selectively oxidized to give either linear or branched 
allyl acetates (Scheme 9). Palladium acetate in a mixture of DMSO and acetic acid (1:1) regioselectively gave the corresponding linear allyl acetates in reasonable yield and with high $E: Z$ selectivity. Palladium acetate bis-sulfoxide complex 19, on the other hand, furnished branched allyl acetates in a similarly regioselective fashion.

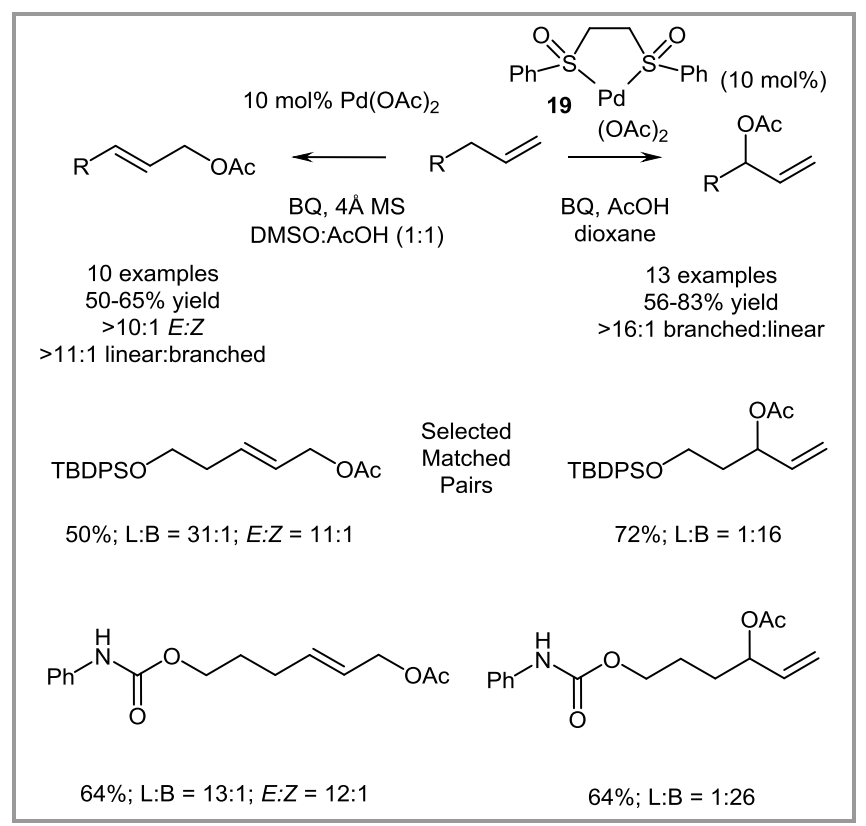

Scheme 9 Tunable regioselectivity in the sulfoxide-promoted allylic oxidation of terminal alkenes.

The bis-sulfoxide ligand was shown to partially decompose under the reaction conditions to generate phenylvinyl sulfoxide. ${ }^{15}$ This commercially available compound could also be employed successfully in place of the bis-sulfoxide and it is likely to be the active ligand in this process. This was the first example of a general, synthetically useful methodology for the selective formation of allyl acetates from terminal alkenes. Only very small quantities of the corresponding methyl ketones were observed under these conditions, making this a valuable complimentary approach to the Wacker oxidation.

As a consequence of this and other recent developments described herein, the palladium(II)mediated allylic oxidation of alkenes has become a powerful synthetic tool, receiving much attention in recent years.

\subsection{Allylic Oxygenation}

In the decade or so since the publication of their original paper, the White group has elaborated upon the scope of their sulfoxide-promoted reaction with some elegant applications of the chemistry. ${ }^{16}$ In 2006 , they demonstrated the ability to rapidly access complex polyoxygenated scaffolds from bulk chemical starting materials with a short, de novo synthesis of differentially protected L-galactose (Scheme 10). ${ }^{16 \mathrm{j}} \mathrm{A}$ homoallyl acetonide was subjected to palladium(II)-mediated allylic oxidation using $p$ methoxybenzoic acid as nucleophile to give the corresponding linear allylic ester in good yield and excellent regio- and stereoselectivity.

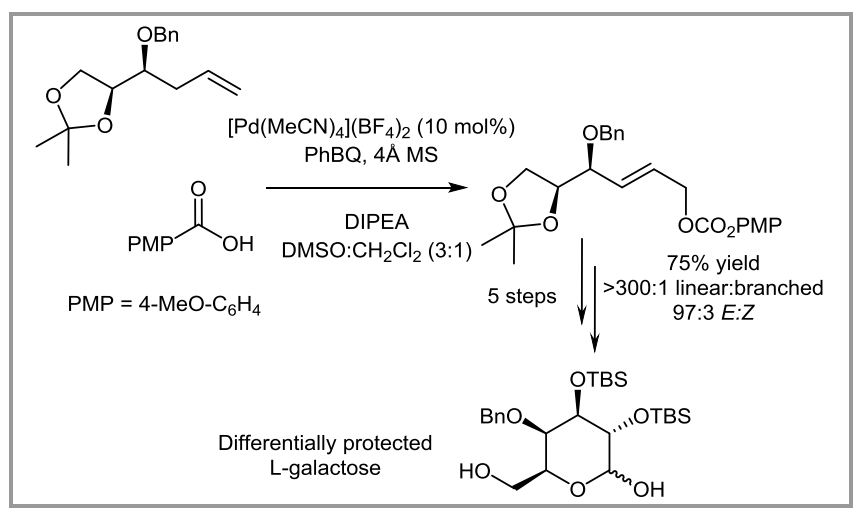

Scheme 10 De novo synthesis of differentially protected Lgalactose by highly regioselective linear allylic oxidation.

Taking advantage of the unique ability of $p$ methoxybenzoates of (E)-2-butene-1,4-diols to undergo highly diastereoselective asymmetric dihydroxylation, ${ }^{17}$ the group rapidly completed the enantioselective synthesis of the hexose framework.

The use of allylic oxidation can offer a more efficient alternative to traditional $\mathrm{C}-\mathrm{C}$ bond forming chemistry. For example, several recent publications from the White group have described successful alternative routes to a variety of synthetic targets, which circumvent traditional olefination reactions. A representative selection of examples is outlined in Scheme 11. In the first case (a), a linear allylic oxidation was carried out on a differentially protected diol, which is readily prepared from protected glycidol in two steps. ${ }^{16 \mathrm{e}}$ The resulting linear allyl benzoate is a key intermediate in the synthesis of (-)-laulimalide, which was previously synthesized by olefination in six steps. ${ }^{18}$ A second example using a similar strategy is given by the three step synthesis of a linear allyl benzoate, a precursor to trans-fused polycyclic ethers present in the brevetoxin scaffold (b). ${ }^{19}$ The efficient three step synthesis from commercially available cyclohexene oxide using allylic oxidation chemistry represents a significant improvement over the previously reported six step olefination route. Finally, anti-1,4-dioxan-2-ones were shown to be highly versatile intermediates that can be readily elaborated to syn-1,2-diols, stereo-defined amino-polyols and syn-pyrans (c). ${ }^{16 \mathrm{c}}$ By tethering the reacting carboxylic acid to a homoallylic alcohol, a carboxylate-chelated inner sphere allylic functionalisation reaction was enabled, giving access to anti-1,4-dioxan-2-ones. The example shown illustrates the utility of this process, shortening the previously reported route to the synpyran intermediate used for preparing Goniodomin A. $^{20}$ The 1,4-dioxan-2-one, prepared by allylic 
oxidation, undergoes an Ireland-Claisen rearrangement to give the desired syn-pyran intermediate, which is isolated as its methyl ester in excellent yield. This improved sequence is three steps shorter than the previously reported approach based on traditional olefination chemistry.

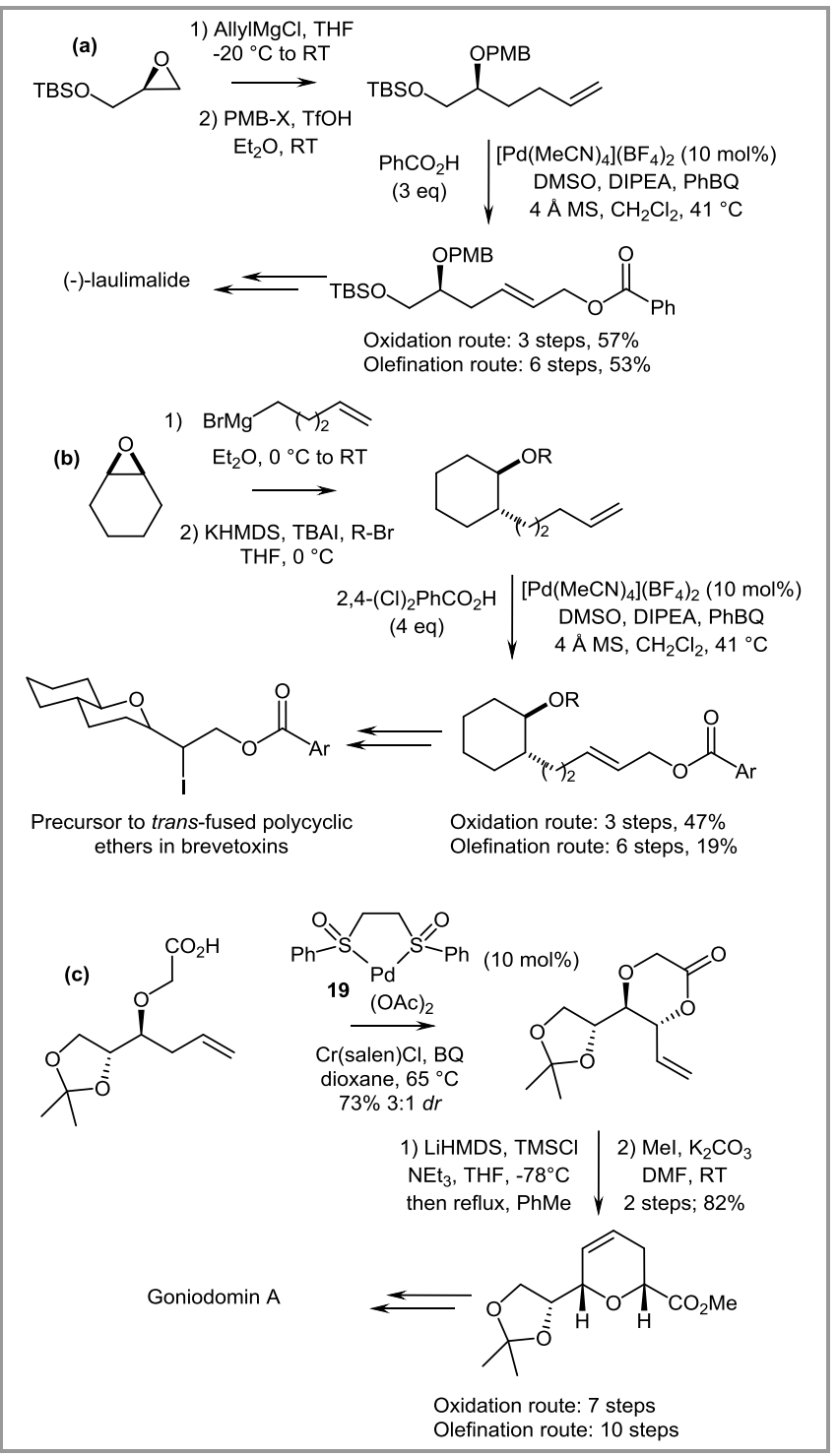

Scheme 11 Allylic oxidation as a more efficient alternative to olefination in natural product synthesis.

Perhaps the most impressive achievement of this chemistry is its successful employment in the total synthesis of 6-deoxyerythronolide B (Scheme 12). ${ }^{16 \mathrm{f}}$ A late-stage intramolecular functionalisation of intermediate $\mathbf{2 0}$ gave access to advanced macrolactone 21a with high diastereoselectivity for the natural epimer. This intermediate was then readily elaborated to give the natural product in three additional steps. Addition of fluoride ion to $\pi$-allyl palladium complexes is known to promote $\pi-\sigma-\pi$-interconversion through occupation of a coordination site on the metal centre. ${ }^{21}$ It was postulated therefore that addition of fluoride to the reaction mixture could alter the stereochemical outcome by disrupting the palladium- carboxylate chelate through which $\mathbf{2 1}$ is presumably formed. Pleasingly, the addition of tetra- $n$ butylammonium fluoride (TBAF) produced the desired effect, resulting in a 1:1.3 mixture of the natural material 21a and its epimer $\mathbf{2 1 b}$.

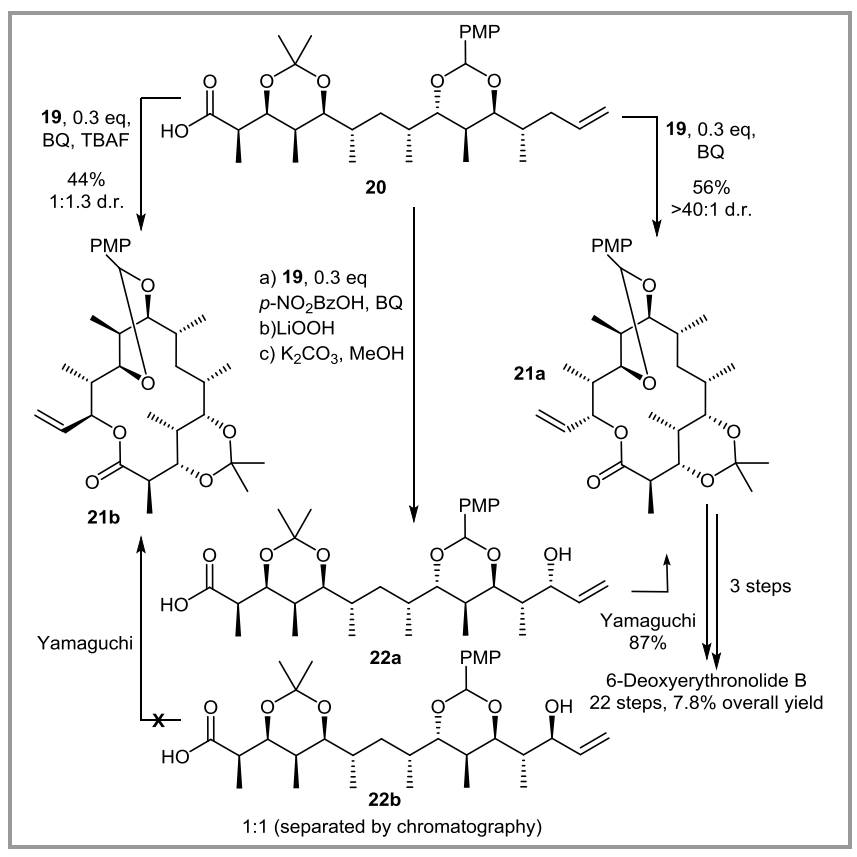

Scheme 12 Total synthesis of 6-deoxyerythronolide B by late-stage C-H oxidation.

The significance of this ability to selectively furnish either one of the two epimers was highlighted when a synthesis of the same intermediates was attempted using more traditional chemistry. Yamaguchi macrolactonisation of allylic alcohol 22a (prepared via a modification of the allylic oxidation chemistry) furnished the expected natural epimer 21a in excellent yield. However, exposure of the opposite diastereomer 22b to the same conditions yielded only oligomers, demonstrating that macrolactone $\mathbf{2 1 b}$ is not accessible via the common traditional approach. By using a tunable late-stage allylic oxidation strategy rather than a macrolactonisation approach, the group were able to circumvent this issue and access both the naturally occurring compound and its unnatural epimer. A similar strategy was later employed to complete the total synthesis of erythromycin. ${ }^{16}$

In more recent years, a number of other groups have begun to take advantage of the high chemo- and regioselectivity that can be achieved using palladium acetate bis-sulfoxide complex 19 (generally referred to as the White catalyst). For example, Malik recently applied this approach to the total synthesis of unnatural iminosugar (-)-castanospermine (Scheme 13). ${ }^{22}$ In this instance, the Cbz nitrogen protecting group provides the nucleophilic oxygen source, which cyclises onto the allylic position in the presence of the White catalyst and a Lewis acid. The resulting oxazolidinone was isolated as a single diastereomer and was readily elaborated to give (-)- 
castanospermine. The use of simple, readily available carbamates as nucleophiles in this reaction is unprecedented and represents an exciting new prospect for future developments. The fact that this reaction doesn't work in the presence of molecular sieves suggests that hydrolysis of the intermediate cationic cyclic carbamate is a key step in this process that drives the reaction to completion.

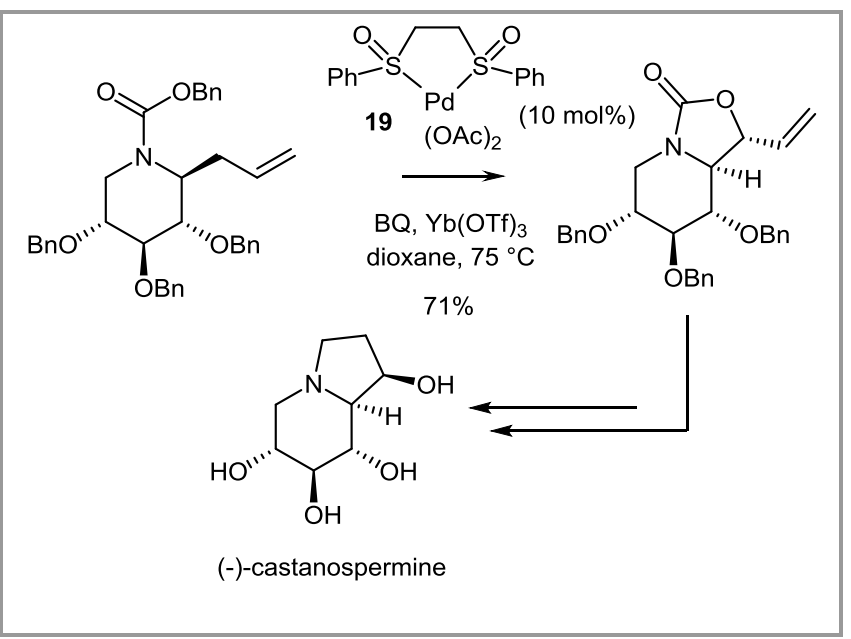

Scheme 13 Total synthesis of (-)-castanospermine using intramolecular allylic oxidation as the key step.

Similarly, the use of the bis(sulfoxide) Pd catalyst 19 to promote an intramolecular oxidative allylic cyclisation to form biologically relevant tetracyclic flavonoids was recently reported by Belani (Scheme 14). ${ }^{23}$ Until relatively recently, oxygen nucleophiles employed in the palladium bis-sulfoxide catalyzed process have all been weakly acidic (typically carboxlates with $\mathrm{pKa}<6$ ). More basic nucleophiles tend to perform less well under these reaction conditions as they are not readily deprotonated and can also deactivate the catalyst. The successful use of phenols $(\mathrm{pKa} \sim 10)$ and even aliphatic alcohols (pKa $\sim 16)^{16 \mathrm{a}}$ as nucleophiles represents an important improvement in the scope of this reaction.

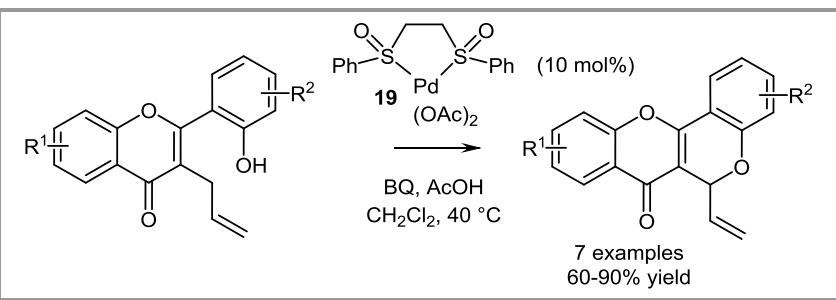

Scheme 14 Tetracyclic flavonoid synthesis by intramolecular oxidative allylic cyclisation.

Several other catalytic systems have been developed for the selective formation of either linear or branched allylic oxidation products (Scheme 15). In 2010, Stambuli reported the linear-selective allylic oxidation of terminal alkenes using thioether ligand 23 (a). ${ }^{24}$ The scope and functional group tolerance of this reaction were comparable to the procedure first published by White, although the reaction times tended to be slightly shorter in this case. In a more comprehensive survey of palladium-sulfide catalysts, Stambuli later demonstrated that the simple and inexpensive tetrahydrothiophene was a highly active and linear-selective ligand (b). ${ }^{25}$ In an effort to move away from the use of stoichiometric quantities of oxidants such as BQ or copper additives, Stahl developed the first catalytic system to achieve aerobic turnover with 4,5-diazafluorenone ligand 24 (c). ${ }^{26}$ This system proved to be highly selective for linear allylic acetates with good yields and functional group tolerance. An earlier example of a direct oxygencoupled allylic oxidation was reported by Kaneda (d) alongside their procedure for the Wacker oxidation (vide infra). ${ }^{27}$ This method uses only $1 \mathrm{~mol} \%$ of palladium catalyst but a high pressure of oxygen is necessary for efficient catalyst turnover. With molecular oxygen as the sole re-oxidant, these systems clearly offer an environmental advantage over other approaches to allylic oxidation.

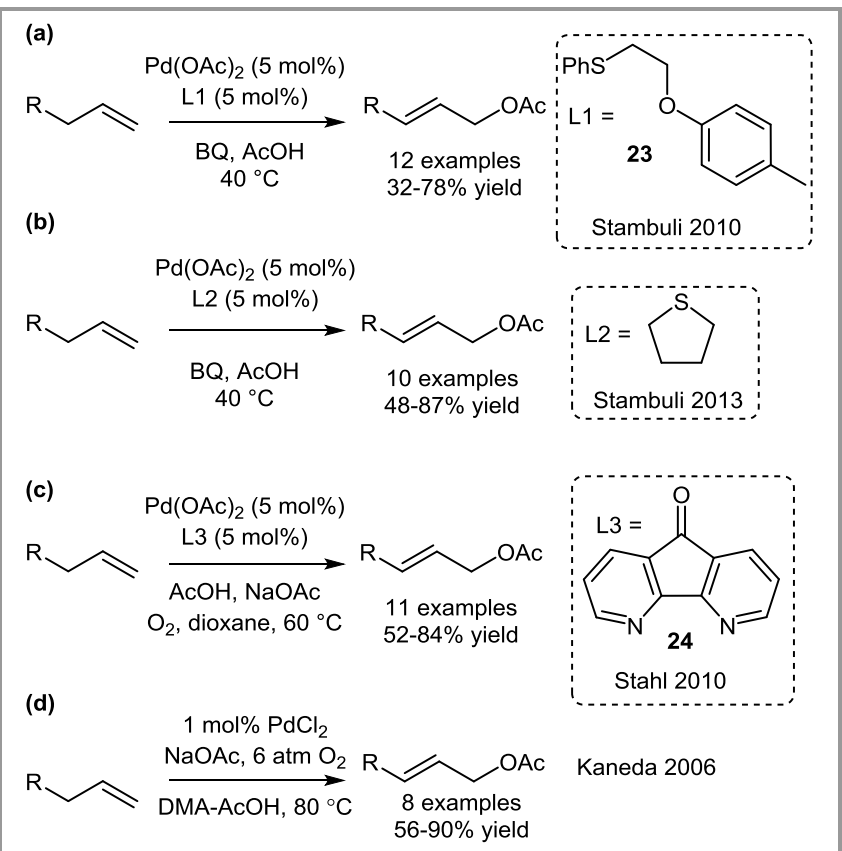

Scheme 15 Catalyst systems for linear-selective allylic oxidation.

More recently, Liu has reported the use of a $\mathrm{Pd} / \mathrm{sox}$ catalyst system that is highly selective for branched allylic esters. ${ }^{28}$ The bulky oxazoline of sox ligand $\mathbf{2 5}$ (Scheme 16) hinders functionalisation at the terminal carbon atom, favouring oxidation at the internal allylic position, which results in the formation of branched products. This development opens up the possibility for ligand-derived stereochemical induction if the $\mathrm{Pd} /$ sox catalyst system could be suitably modified. Notwithstanding, at the time of writing this catalyst system is the only alternative to the White catalyst for selective formation of branched allylic esters. 


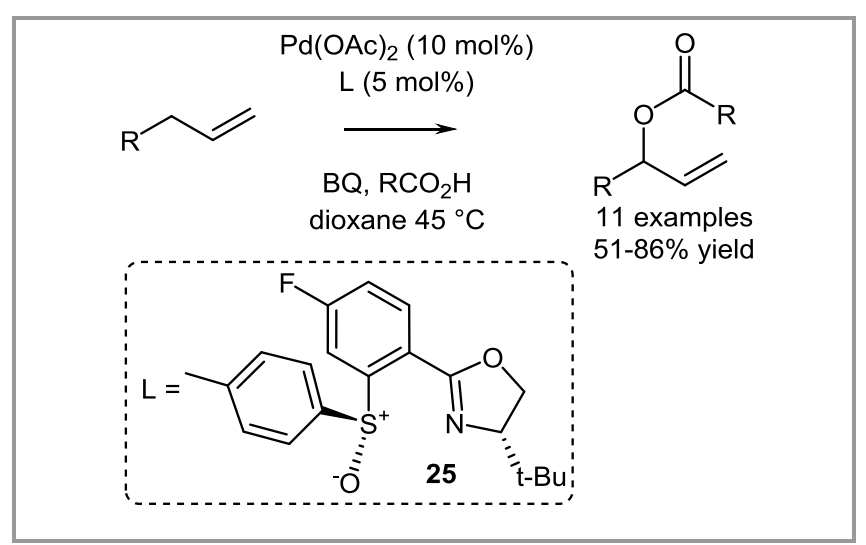

Scheme 16 A Pd/sox catalyst system for the branch-selective synthesis of allylic esters.

As mentioned above, allylic alcohols and esters are common starting materials in a variety of different reactions. A methodology that enabled the one-pot conversion of simple alkenes into intermediate substrates that can then undergo further manipulations would therefore be a powerful synthetic tool for rapidly accessing complex molecular architecture. Hartwig recently demonstrated the potential of this approach by employing a one-pot allylic oxidationenantioselective functionalisation reaction (Scheme 17). ${ }^{29}$ Taking inspiration from the success of the onepot iridium-catalysed borylation-Suzuki coupling of aromatic C-H bonds, ${ }^{30}$ the group sought to develop a method for iridium-catalysed allylic functionalisation in which the intermediate linear allylic ester was formed in situ from a terminal alkene. To this end, it was therefore necessary to develop a mild, neutral allylic oxidation reaction in which neither the nucleophile nor the co-oxidant would interfere with the iridium-catalyzed functionalisation step. In order to achieve this, the group came up with the creative solution of using tert-butyl perbenzoate as both the co-oxidant and source of nucleophilic carboxylate. In the presence of palladium acetate and Stahl's 4,5diazafluorenone ligand $\mathbf{2 4}$, linear alkenes were selectively oxidized to their corresponding linear allyl benzoates in good yield, with the tert-butyl perbenzoate successfully providing both nucleophile and oxidant. The intermediate allyl benzoates proved to be adequate electrophiles in the iridium-catalysed step, undergoing rapid transformation to the desired functionalised products in good yield and high enantioselectivity without the need for isolation. A variety of terminal alkenes were successfully functionalised using this one-pot procedure with anilines, benzylamines, phthalimides and benzimidazole to give the corresponding branched allylic amines. Oxygen, sulfur and even carbon nucleophiles were also successfully employed to give branched allyl ethers/silyl ethers, sulfones and malonates respectively in similar high yield and enantioselectivity. The strength of this methodology was then demonstrated with the short, enantioselective synthesis of two biologically active compounds (-)-
$(R)$-vigabatrin and (-)-(R)-angustureine in three and four steps respectively from commercially available terminal alkenes.

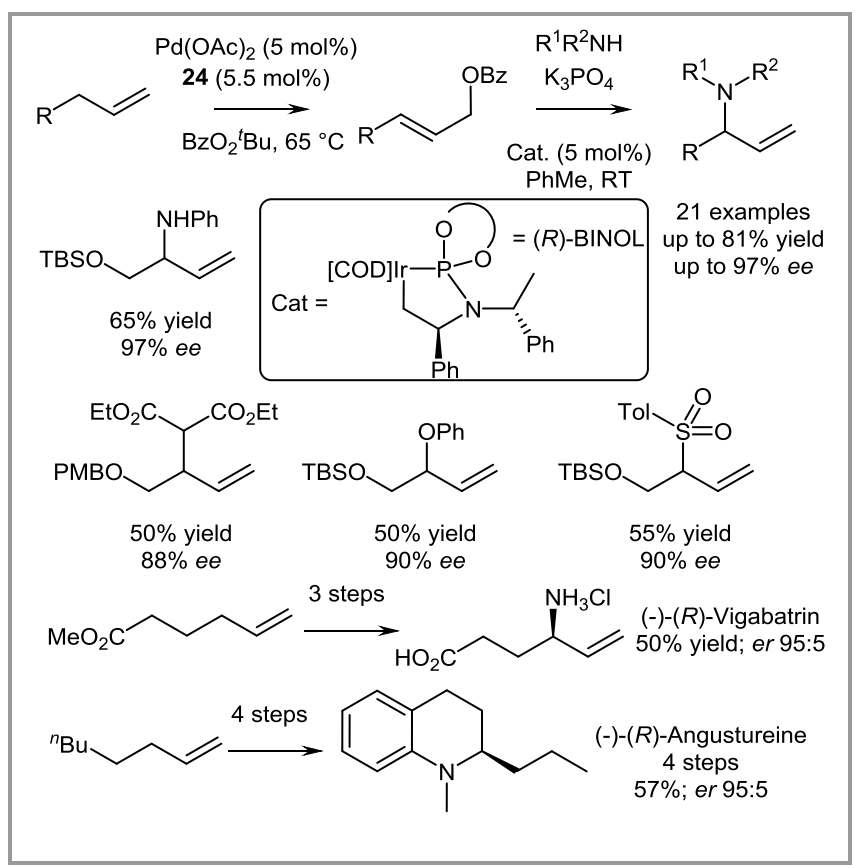

Scheme 17 One-pot allylic oxidation-functionalisation by in situ generation of a linear allyl benzoate electrophile.

Taking this idea a step further, Hartwig then demonstrated an iterative approach to $(1, \mathrm{n})$ difunctionalised chiral fragments using this one-pot strategy (Scheme 18). After an initial allylic oxidation-functionalisation of a linear alkene, the resulting chiral product can be further elaborated by hydroboration of the allyl group and Suzuki coupling with a suitable bromoalkene. This homologated intermediate can then be further subjected to a second one-pot allylic oxidation-functionalisation procedure to give $(1, \mathrm{n})$-difunctionalised chiral fragments. In this manner, several $(1, \mathrm{n})$-difunctionalised $(\mathrm{n}=3-8)$ chiral compounds were readily accessible, bearing different combinations of oxygen, nitrogen, sulfur and carbon substituents. 


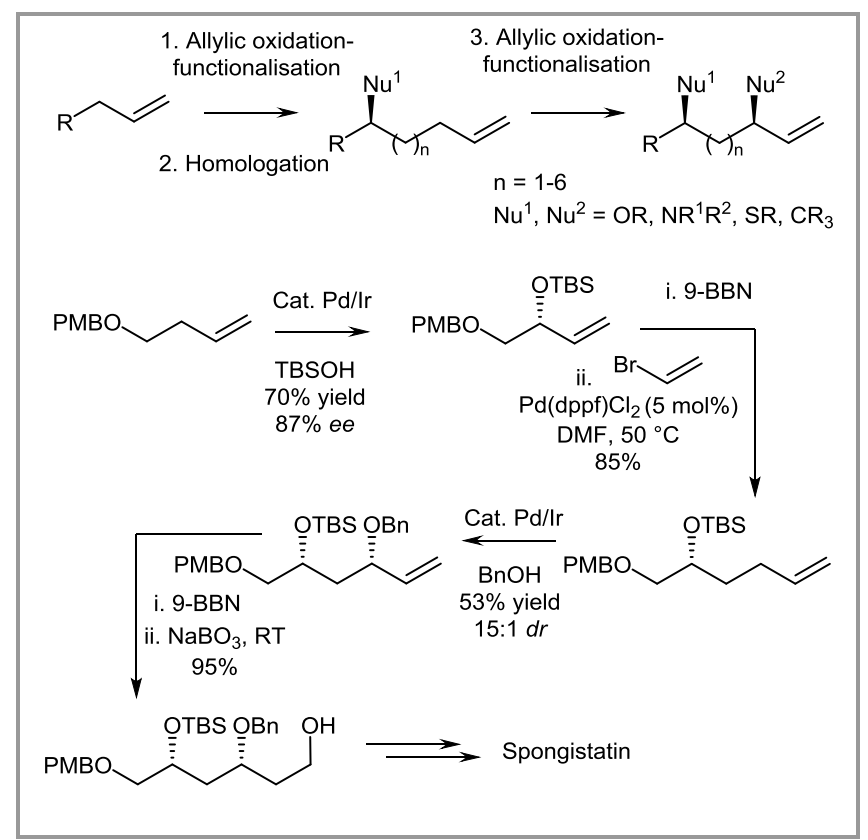

Scheme 18 Iterative approach to $(1, n)$-difunctionalised chiral fragments using the one-pot allylic oxidation-functionalisation reaction.

To showcase the ability of this strategy to furnish densely functionalised chiral compounds, the group synthesised a differentially protected polyol intermediate, applicable to the synthesis of the Spongistatins. $^{31}$ A first allylic oxidationfunctionalisation of protected but-3-en-ol with tertbutyldimethylsilanol gave a chiral TBS-protected allylic alcohol. Homologation via hydroboration and Suzuki coupling with vinyl bromide, followed by a second allylic oxidation-functionalisation with benzyl alcohol furnished a differentially protected triol in excellent yield and diastereoselectivity. A second hydroboration and oxidation then completed the synthesis of the 1,2,4,6-tetraol natural product precursor in just four steps from bulk starting materials.

cis-Vinylsilanes have recently been shown to undergo regioselective allylic oxidation to give branched products without the need for an external ligand (Scheme 19). ${ }^{32}$ The geometry of the oxidized vinylsilanes is dependent on the nature of the cooxidant used. With BQ as oxidant, the transvinylsilane is isolated as a single isomer, whereas with $\mathrm{PhI}(\mathrm{OAc})_{2}$ the $c i s$-product predominates.

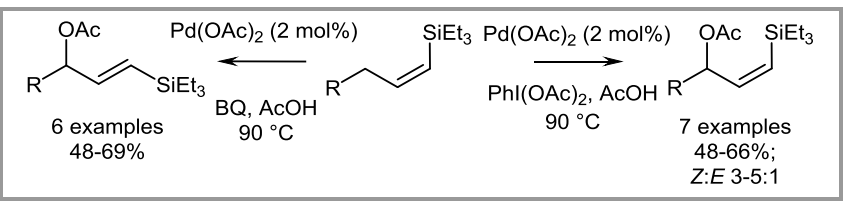

Scheme 19 Co-oxidant dependent product distribution in the palladium-catalysed allylic oxidation of cis-vinylsilanes.

This is rationalized by slow C-O bond-forming reductive elimination from the $B Q-\pi-$
allylpalladium(II) complex allowing the system to isomerise to the more thermodynamically stable transproduct. Oxidation with $\mathrm{PhI}(\mathrm{OAc})_{2}$, a more powerful oxidant, is thought to result in faster reductive elimination from the presumed $\pi$-allylpalladium(IV) complex. This significantly reduces but does not completely eliminate this isomerization. Interestingly, the related trans-vinylsilanes were completely unreactive under these conditions.

An intramolecular version of this reaction was developed for cis-vinylsilanes bearing a tethered aliphatic alcohol, giving rise to cyclic ethers (Scheme 20). A range of simple $\alpha$-substituted alcohols underwent this intramolecular allylic etherification to give tetrahydrofuran and tetrahydropyran products in reasonable yield.

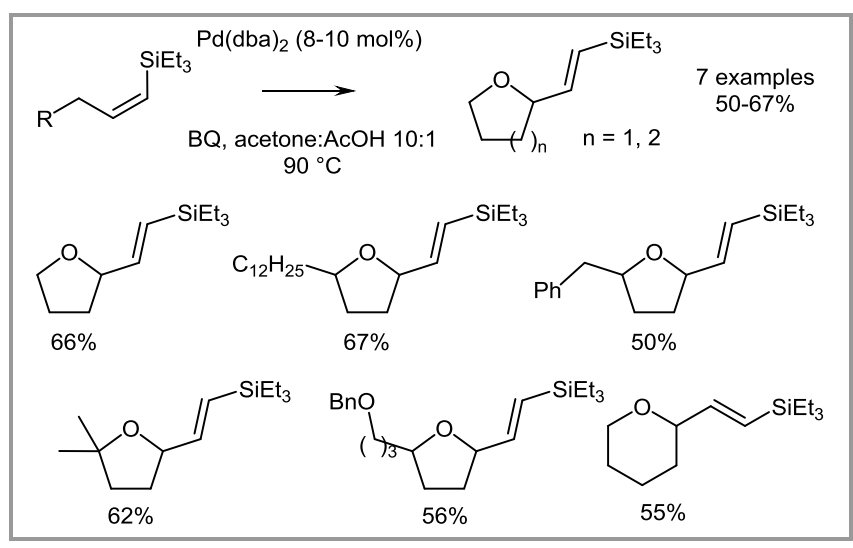

Scheme 20 Intramolecular allylic etherification of cis-vinylsilanes.

Jiang recently reported the first use of water as a nucleophile in the palladium-catalysed allylic oxidation of alkenes (Scheme 21). ${ }^{33}$ Allyl arenes are oxidized to the corresponding linear allylic alcohols in the presence of palladium chloride and water, using DDQ as co-oxidant. These products are readily oxidized by a second equivalent of DDQ to give $(E)$ alkenyl aldehydes in excellent yield.

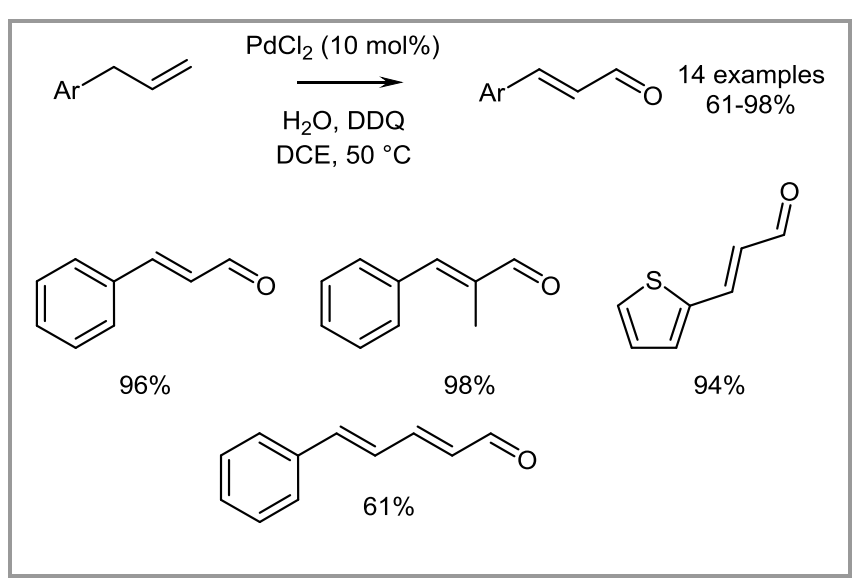

Scheme 21 Synthesis of $(E)$-alkenyl aldehydes by allylic oxidation with water as nucleophile. 
Labeling experiments confirmed that water was the source of nucleophilic oxygen, attacking an intermediate $\pi$-allylpalladium species. The additional activating effect of conjugation appears to be key in this process, as simple aliphatic alkenes such as 1decene were unreactive.

\subsection{Allylic Amination}

The construction of carbon-nitrogen bonds is heavily relied upon in synthetic and medicinal chemistry as a method for coupling of pre-elaborated fragments and introducing molecular complexity. ${ }^{34}$ Many traditional approaches to carbon-nitrogen bond formation rely upon multiple step oxidation/reduction sequences that are often incompatible with a variety of functionality and therefore require suitable protecting group strategies. As a result, methods that achieve the direct oxidative amination of simple hydrocarbons have received much attention in recent years. ${ }^{35}$

The White group first extended the scope of their bissulfoxide promoted allylic oxidation reaction to include allylic amination in 2007 (Scheme 22). ${ }^{36} \mathrm{~N}$ tosyl carbamates were selected as a nitrogen source, reasoning that they are sufficiently acidic $(\mathrm{pKa} \sim 3.7)^{37}$ so as to be deprotonated by the palladium acetate counterion, but are unlikely to interfere with the electrophilic C-H cleavage step. Regeneration of the catalyst then proceeds during reduction of $\mathrm{BQ}$ to hydroquinone in the re-oxidation of $\mathrm{Pd}(0)$ to $\mathrm{Pd}(\mathrm{II})$. By tethering the $N$-tosyl carbamate at the homoallylic position of a terminal alkene, an intramolecular oxidative allylic amination reaction proceeded as envisaged in the presence of bis-sulfoxide palladium complex 19 and 2-phenyl-p-benzoquinone (PhBQ). The resulting oxazolidinones were furnished in high yield with varying levels of diastereoselectivity for the anti-product. Substrates with one branching element adjacent to the $N$-tosyl carbamate produced the best combination of chemical yield and anti-selectivity. Linear substrates performed slightly better in terms of yield, but suffered from a lack of diastereoselectivity, whilst tertiary branched substrates were highly antiselective but gave much lower yields.

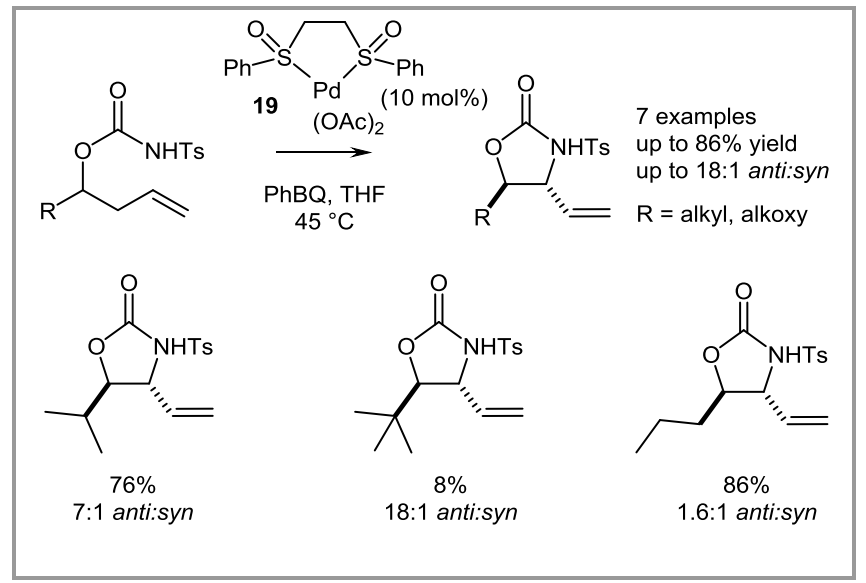

Scheme 22 The first example of an intramolecular allylic amination reaction to give anti-oxazolidinones.
Interestingly, an experiment with stoichiometric bissulfoxide palladium acetate complex 19 resulted in the slow formation of product, but with no observation of the intermediate $\pi$-allylpalladium species. On switching to $\operatorname{Pd}(\mathrm{TFA})_{2}$, which has a less basic counterion, the $\pi$-allylpalladium intermediate could be observed as the major species present by ${ }^{1} \mathrm{H}$ NMR, with no detectable product formation. Addition of external acetate to this complex then resulted in a similar conversion to product as seen with palladium acetate, suggesting that deprotonation of the $N$-tosyl carbamate nucleophile is indeed an important step in this transformation.

The anti-oxazolidinone products furnished by this reaction are readily hydrolysed to give syn-1,2aminoalcohols, which are useful intermediates in a variety of medicinally relevant chemistry. To demonstrate the utility of this intramolecular allylic amination reaction, the group conducted a streamlined synthesis of a precursor to the aminosugar (-)- $\mathrm{N}$ acetyl-O-methyl acosamine (Scheme 23). The required chiral $N$-tosyl carbamate was readily accessed from a readily available chiral aldehyde using standard chemistry. The key allylic amination reaction then proceeded as planned with high diastereoselectivity for the anti-oxazolidinone product, completing the synthesis in just three steps without the need for functional group manipulations (FGM). In contrast, the previous route to this intermediate required six steps and involved the use of two FGMs, ${ }^{38}$ which clearly demonstrates the benefits of the allylic amination chemistry.

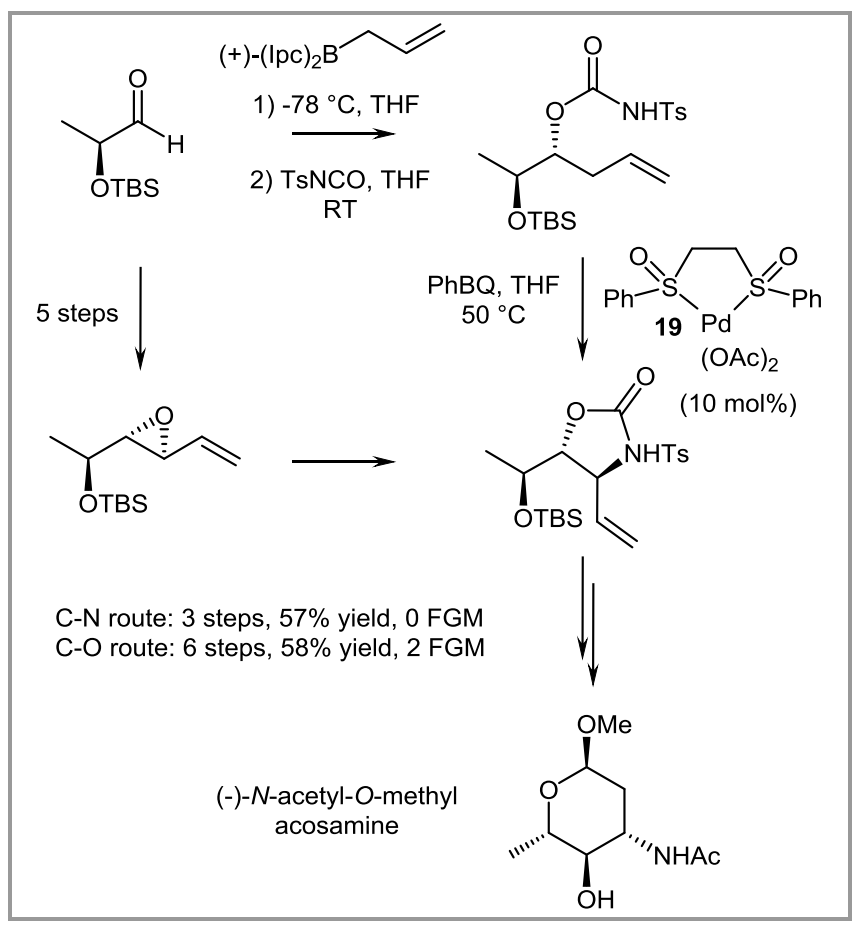

Scheme 23 Streamlined synthesis of a medicinally relevant aminosugar precursor using palladium-mediated allylic amination chemistry. 
Further extending the scope of this reaction, the White group later demonstrated that the vinyl oxazolidinone products obtained from the intramolecular allylic amination could undergo an in situ oxidative Heck reaction $^{39}$ to give homophenylalanine derivatives (Scheme 24). ${ }^{40}$ Shorter reaction times for the amination step were observed when switching from $N$-tosyl carbamates to the analogous $o$-nitrosulfonyl (Ns) compounds. This enabled the concentrations of active catalyst to remain high enough for the oxidative Heck step to proceed in one pot, without the need for isolation or additional palladium source.

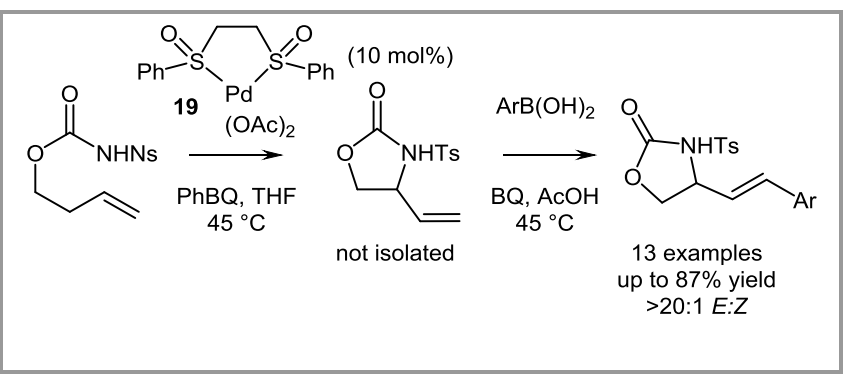

Scheme 24 Sequential allylic amination/oxidative Heck coupling to give homophenylalanine derivatives.

A similar approach was employed by Liu and coworkers to achieve the intramolecular allylic cyclisation of $N$-tosyl amides bearing a terminal olefin to give 5-, 6- and 7-membered heterocycles (Scheme 25). ${ }^{41}$ Treatment of $N$-tosyl amides with palladium acetate in the presence of (salen) $\mathrm{Cr}(\mathrm{III}) \mathrm{Cl}$ as cooxidant furnished pyrrolidinones in good yield. Intriguingly, addition of a Brønsted base (NaOBz) resulted in a complete change in the regiochemistry, giving rise to the analogous azepinones. A range of modified $N$-tosyl amide substrates were successfully employed, resulting in azepinones, oxazepinones, and even piperidinones from the appropriately truncated starting material.

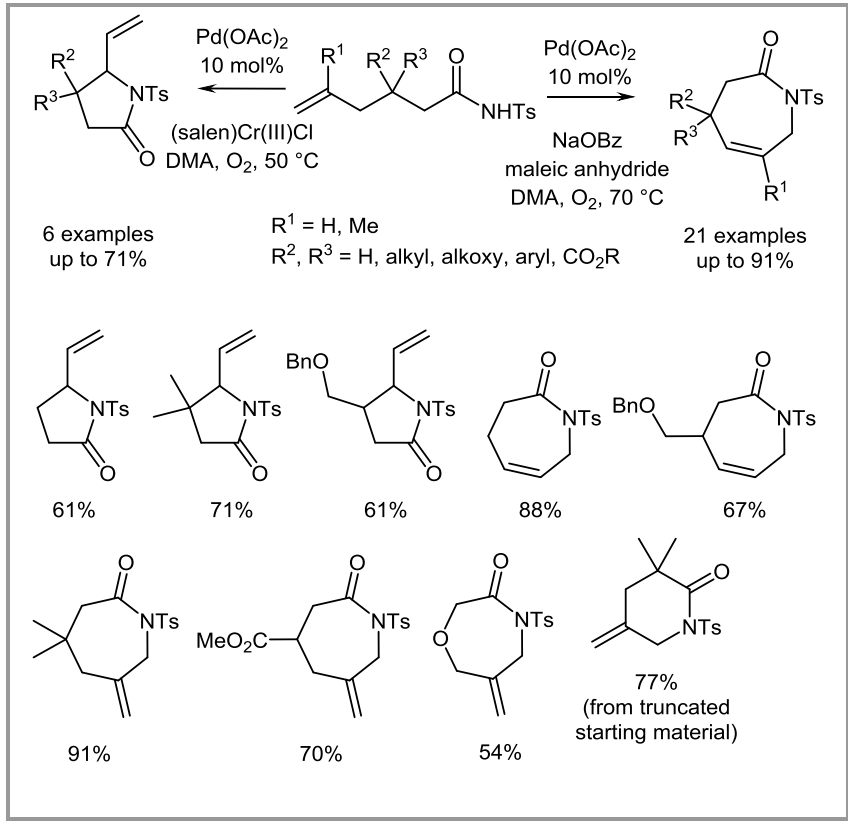

Scheme 25 Intramolecular allylic amination to give 5-, 6- and 7membered nitrogen heterocycles.

Despite the advances being made in the intramolecular allylic amination reaction, the development of an intermolecular variant remained a significant challenge. The original conditions that successfully enabled the intramolecular cyclisation of $N$-tosyl carbamates were ineffective in the intermolecular reaction, due to low concentrations of deprotonated nitrogen nucleophile with endogenous acetate as the only source of base. White first circumvented this issue by employing an activating Lewis acid cocatalyst in the form of (salen) $\mathrm{Cr}$ (III)Cl, although this procedure suffered from poor functional group tolerance and promoted isomerization of electrondeficient alkene substrates. ${ }^{42}$ An improved procedure was later published using an exogenous Brønsted base to increase the concentrations of deprotonated nitrogen nucleophile in the reaction mixture. ${ }^{43}$ The nature of the base had to be carefully balanced between its ability to deprotonate the substrate and its tendency to coordinate to the electrophilic palladium(II) catalyst, leading to deactivation. Optimum conversion to the desired linear allylic amine was observed with $N, N$-diisopropylethylamine (DIPEA) as base and $N$-tosyl methylcarbamate as nucleophile (Scheme 26). A range of terminal alkenes were successfully oxidized under these conditions to give the corresponding protected linear allylic amines in good yield, with significantly improved functional group tolerance. 


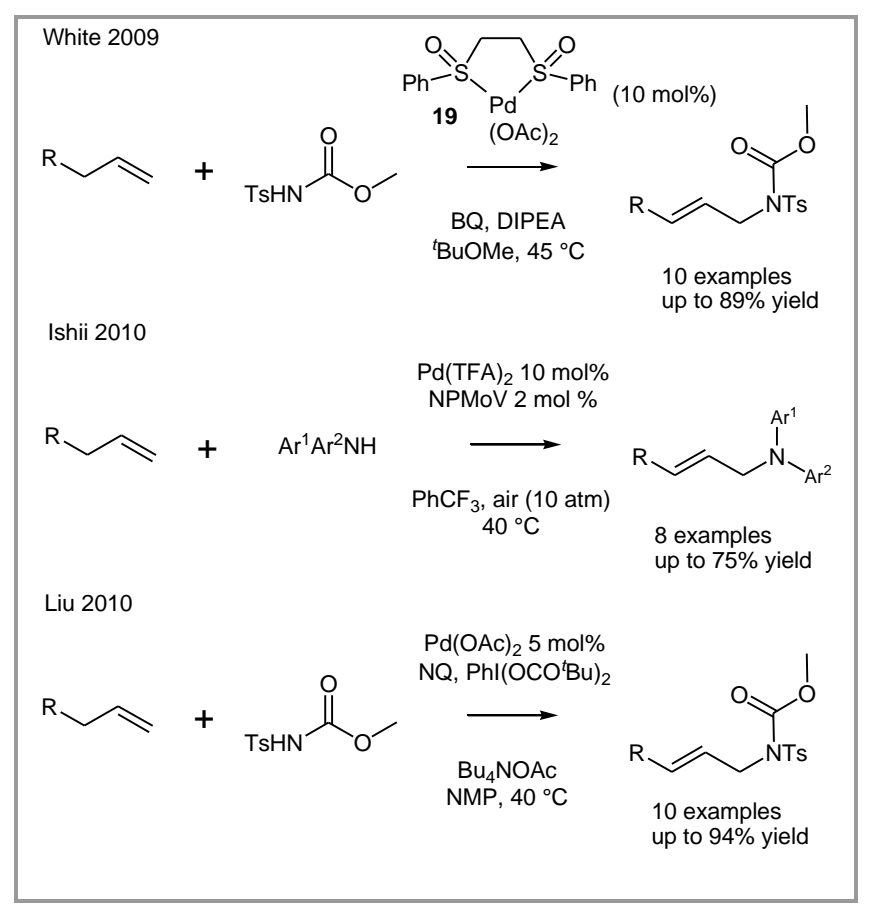

Scheme 26 Methods for the intermolecular allylic amination of olefins.

Two other groups also published procedures for the intermolecular allylic amination of olefins at around the same time. Ishii demonstrated that terminal olefins undergo linear-selective allylic amination with diaryl amines in the presence of $\mathrm{Pd}(\mathrm{TFA})_{2}$ and a molybdovanadophosphate salt NPMoV. ${ }^{44}$ However, the fact that this reaction is limited in its scope to the use of diaryl amines significantly reduces its impact as a useful synthetic tool. $N$-Tosyl carbamates were also successfully employed in the intermolecular allylic amination reaction by Liu. ${ }^{45}$ The strong oxidizing conditions used in this process open up the possibility that these reactions proceed through a $\mathrm{Pd}(\mathrm{IV})$ intermediate. Detailed mechanistic investigations were carried out to probe this hypothesis, finding that the naphthoquinone (NQ) plays a key role in determining the reaction pathway. However, as products were also observed in the absence of either the naphthoquinone or $\mathrm{PhI}(\mathrm{OPiv})_{2}$, the presence or absence of a $\mathrm{Pd}(\mathrm{IV})$ intermediate in this cycle remains somewhat uncertain.

The versatility of the $N$-tosyl carbamate-protected amine products was demonstrated by White via selective deprotection sequences (Scheme 27). For instance, either of the two protecting groups could be selectively removed to give two different monoprotected primary amines (a). Similarly, removal of both protecting groups sequentially gives the free amine (b). Finally, removal of the tosyl group, followed by reduction of the carbamate gives rise to the $N$-methyl derivative (c). The ease with which these intermediates can be manipulated to give several complementary building blocks makes them attractive components for synthesis. ${ }^{46}$

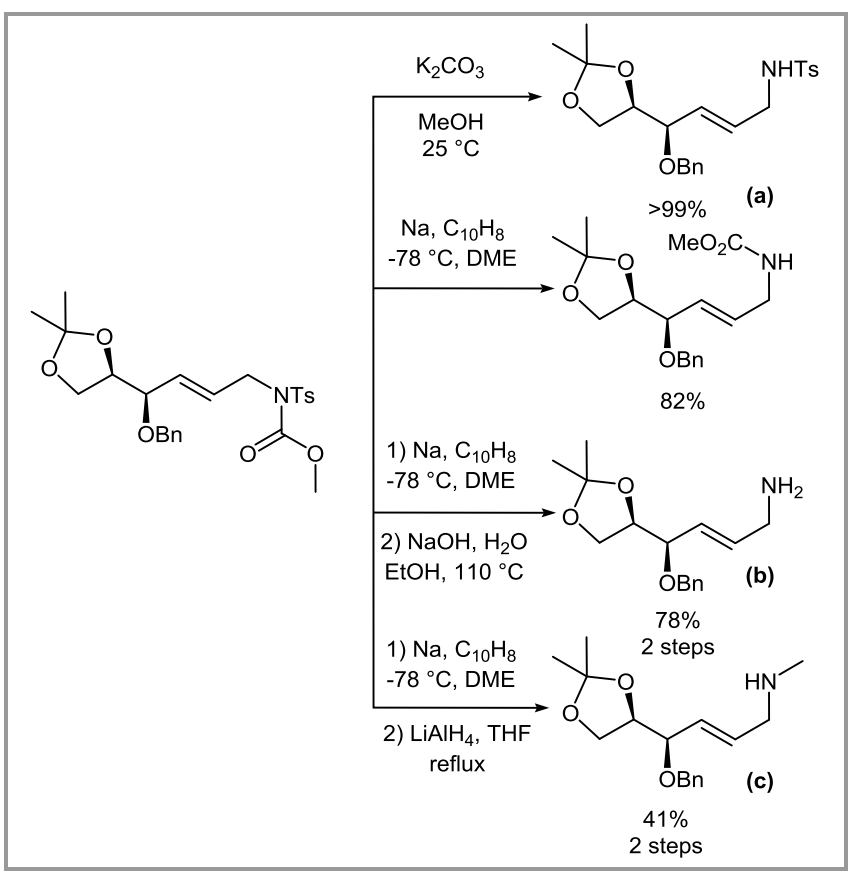

Scheme $27 N$-tosyl carbamates as versatile building blocks.

\subsection{Allylic Functionalisation with Other Nucleophiles}

The extension of palladium(II)-mediated allylic oxidations of olefins to include a broader range of nucleophiles is currently an area of considerable interest. In addition to the traditional $\mathrm{C}-\mathrm{O}$ and $\mathrm{C}-\mathrm{N}$ bond forming processes described above, a number of recent publications involve the introduction of, inter alia, carbon, fluorine and boron to the allylic position of otherwise unactivated olefins. Herein, we summarise some of the significant recent developments in this nascent field.

The White group first described the oxidative allylic alkylation of activated olefins in 2008, using their bissulfoxide palladium complex and methyl nitroacetate as nucleophile (Scheme 28), with 2,6dimethylbenzoquinone (DMBQ) as a co-oxidant. ${ }^{47}$ Several allyl arenes containing different combinations of electron-donating and withdrawing groups were successfully alkylated under these conditions. orthoSubstituted compounds were well tolerated, as were reactive substituents such as triflate, which are often incompatible with palladium-catalysed processes. The activity of the phenyl bis-sulfoxide ligand 19 was shown to deteriorate over time in this reaction through competitive binding of DMSO. The bis-sulfoxide ligand was modified to increase its $\sigma$-dononating properties by replacing the phenyl substituents with benzyl groups. This modified ligand $\mathbf{2 6}$ was sufficiently active to allow the extension of the scope of the allylic alkylation reaction to include simple unactivated olefins. ${ }^{48}$ A number of different substrates were successfully alkylated with a nitroketone as the nucleophile, with excellent functional group tolerance. Proximal stereocentres were preserved, even at an epimerisable position adjacent to a carbonyl. The 
ability of this reaction to introduce new $\mathrm{C}-\mathrm{C}$ bonds at the allylic position of unactivated olefins makes it an orthogonal oxidative approach to the widely used Tsuji-Trost alkylation. ${ }^{6}$

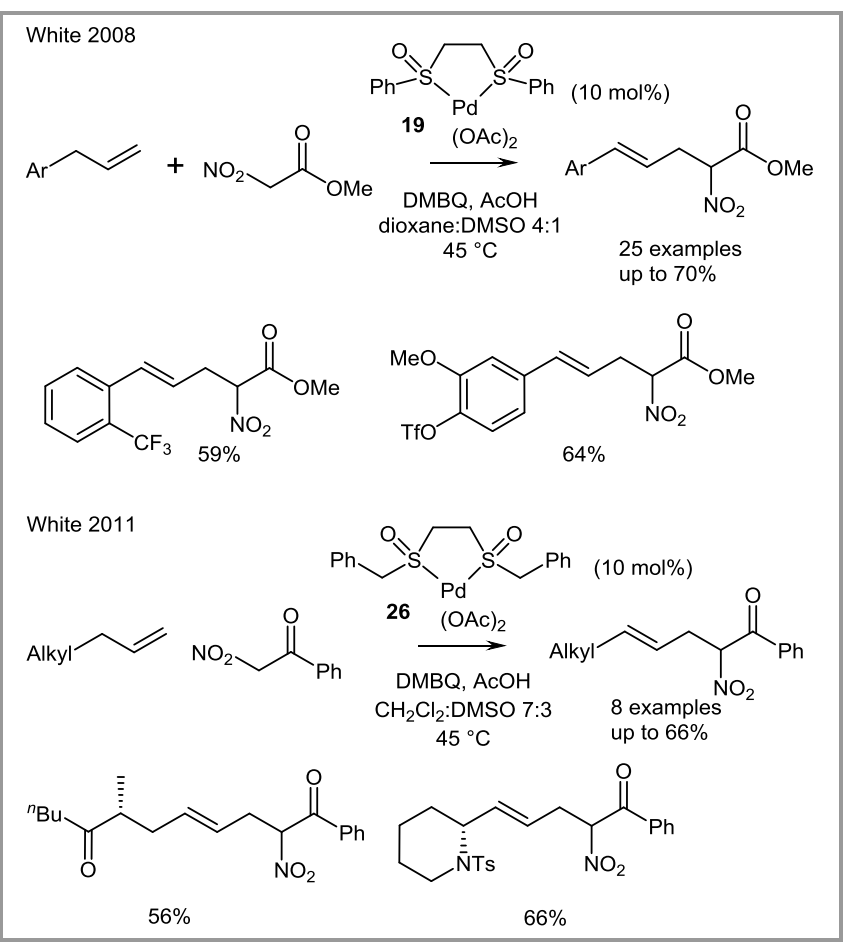

Scheme 28 The first examples of oxidative allylic alkylation of olefins.

More recently, Trost has developed a method for the asymmetric allylic alkylation of 1,3-diketones. ${ }^{49} \mathrm{~A}$ range of allyl arenes underwent enantioselective alkylation in the presence of palladium acetate and phosphoramidite ligand 27 with a 1,3-diketone as the nucleophile (Scheme 29). The alkylated products are furnished in good yields with reasonable levels of enantioselectivity and can accommodate electronwithdrawing but not electron-donating substituents. ortho-Substitution significantly decreased the yield and enantioselectivity. The reaction was also incompatible with certain functionality, such as the strongly coordinating nitrile group and for reasons that are not immediately apparent, ketones.

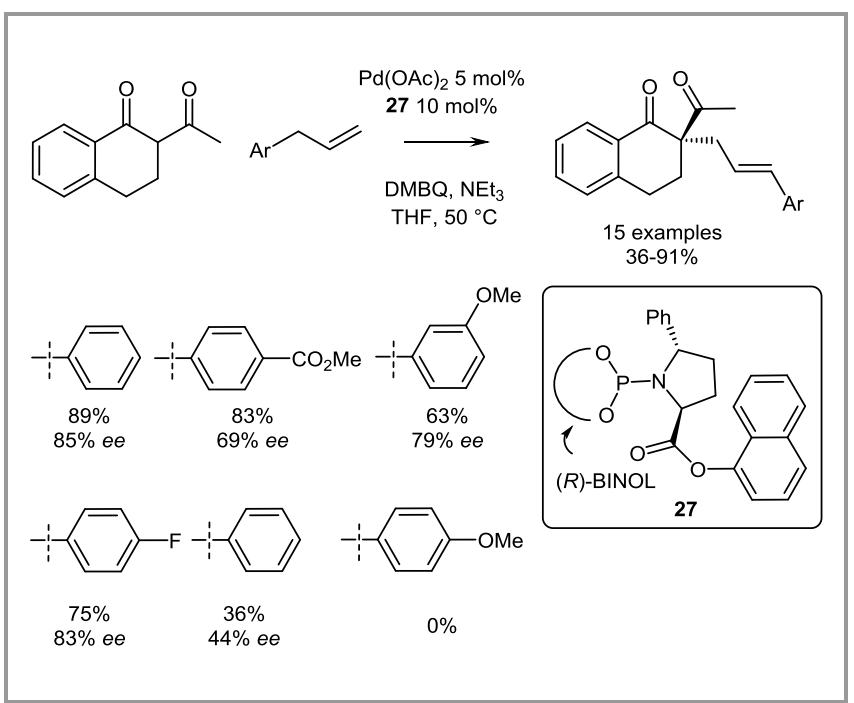

Scheme 29 Enantioselective oxidative allylic alkylation of activated alkenes with 1,3-diketones.

Phosphoramidite 27 was also shown to be a competent ligand in the more traditional $\mathrm{Pd}(0)$-catalysed enantioselective allylic alkylation reaction, providing two complementary approaches to these functionalised products.

Two very similar publications have recently described the allylic arylation of alkenes with polyfluorobenzenes as nucleophiles (Scheme 30). This dehydrogenative coupling was achieved in the presence of silver additives to give the corresponding linear functionalised products in good yield. Conditions developed by Jiang ${ }^{50}$ enabled the efficient functionalisation of unactivated alkenes with pivalic acid (PivOH) as an additive, which presumably facilitates allylic $\mathrm{C}-\mathrm{H}$ cleavage (a). The conditions employed by Yang (b) ${ }^{51}$ were less successful in the oxidation of unactivated alkenes, resulting in mixtures of the desired allylic arenes and Heck-type products. A wider range of poly halogenated nucleophiles were exemplified under Yang's conditions however, with an electron deficient, but otherwise unactivated chlorobenzene providing an interesting example. 


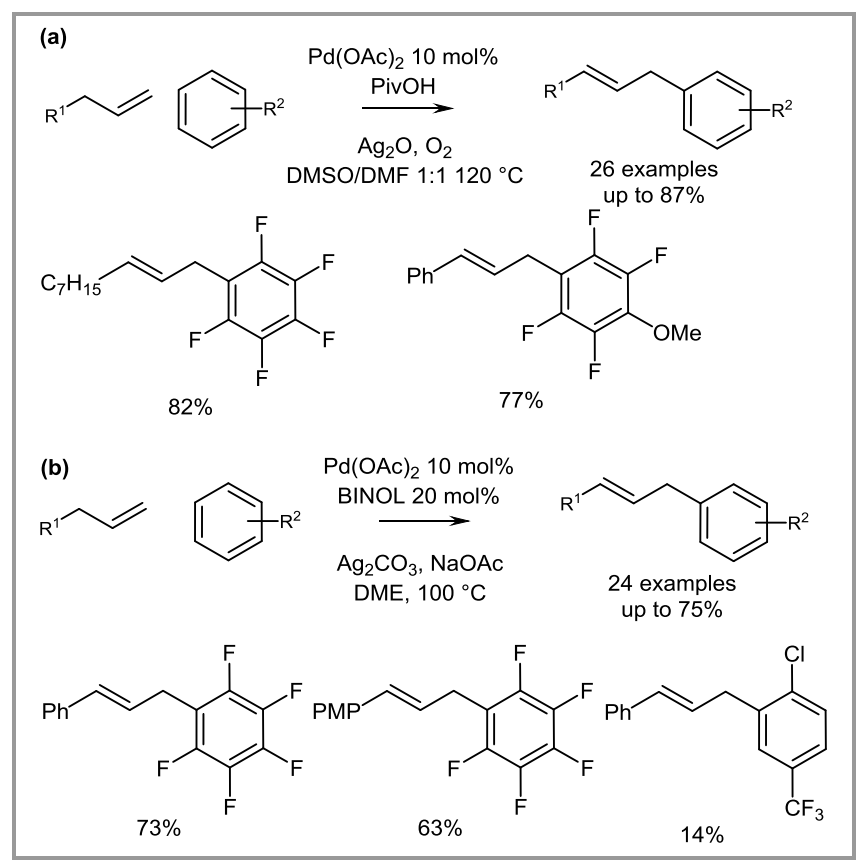

Scheme 30 Allylic arylation with polyfluorobenzenes in the presence of silver additives.

Carbon monoxide was successfully employed as a nucleophile by Jiang to affect the first allylic carbonylation reaction (Scheme 31). ${ }^{52}$ This approach furnishes $\beta, \gamma$-unsaturated esters in good yield and with high selectivity for the linear products.

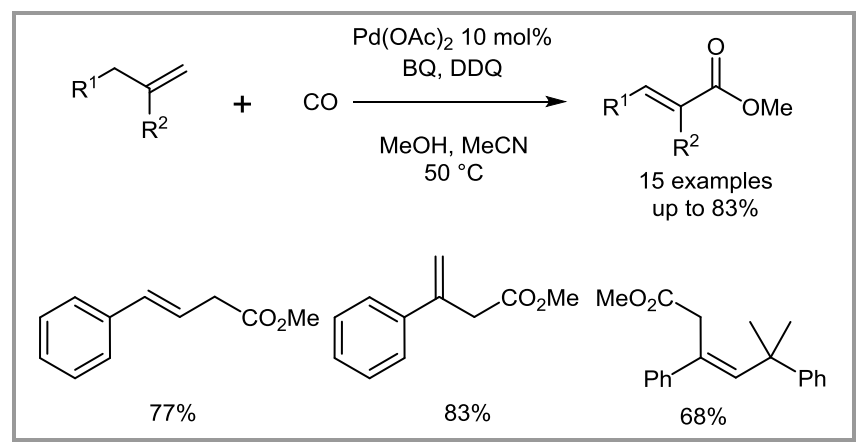

Scheme 31 The first carbonylative allylic oxidation of olefins.

The development of methods for the late-stage introduction of fluorine to bioactive compounds is of paramount importance in medicinal chemistry. ${ }^{53}$ Incorporation of fluorine at the metabolically labile allylic position has the potential to greatly improve ADME/PK properties of alkene-containing drug-like compounds. Doyle recently reported the first oxidative allylic fluorination of alkenes using the benzyl variant of the White ligand and $\mathrm{Et}_{3} \mathrm{~N} \cdot 3 \mathrm{HF}$ in the presence of a catalytic amount of (salen) $\mathrm{CrCl}$ (Scheme 32). ${ }^{54}$ The reaction proceeds in modest yield, but with good regioselectivity and functional group tolerance without the need for rigorous exclusion of water or air. To demonstrate the potential for latestage incorporation of fluorine, the group successfully applied this reaction to a complex steroid scaffold, furnishing the allylic fluoride in excellent yield.

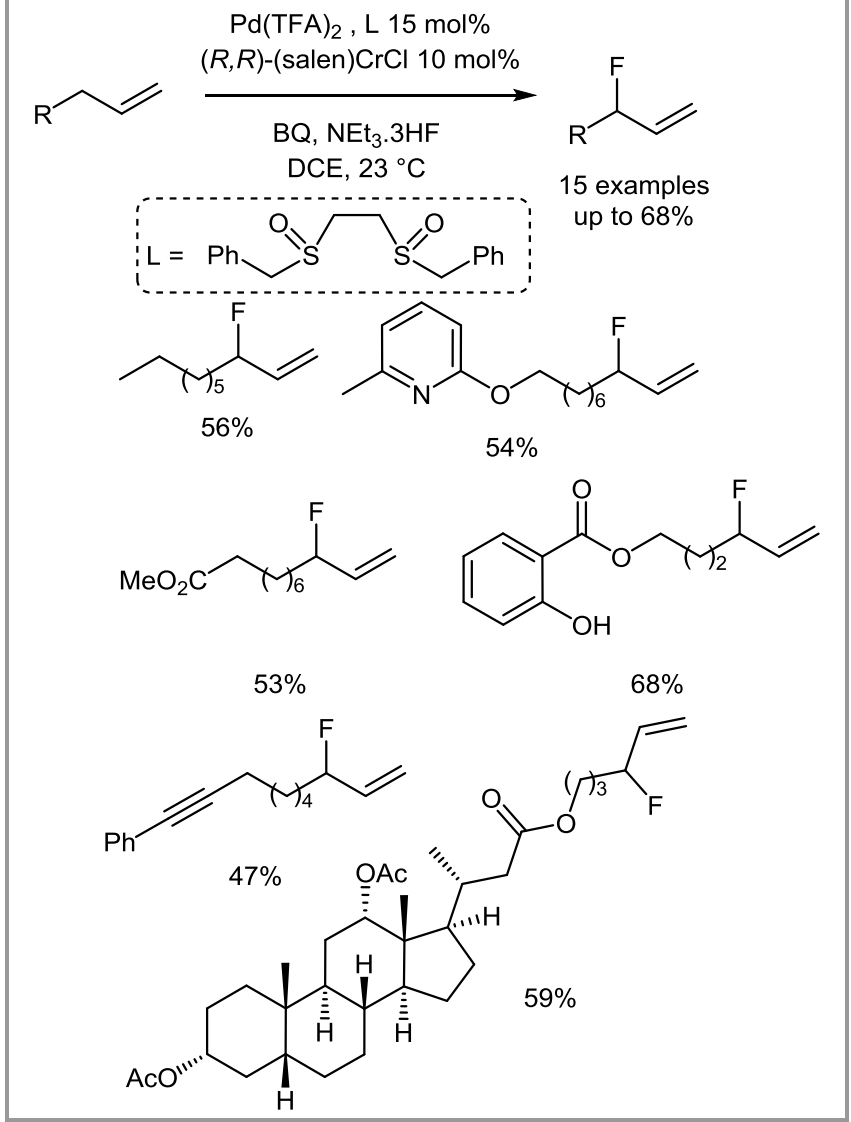

Scheme 32 Oxidative allylic fluorination, with the potential for late-stage functionalisation of drug-like compounds.

Very recently, the first example of an oxidative allylic borylation of alkenes with palladium(II) has been described by Szabó (Scheme 33). ${ }^{55}$ This represents a significant achievement as allyl boronates are prone to rearrangement to the more thermodynamically stable vinyl species $^{56}$ and are particularly sensitive to oxidizing reagents. Exocyclic alkenes can undergo allylic borylation in the presence of $\operatorname{Pd}(\mathrm{TFA})_{2}$ and 2,6dimethyl benzoquinone (DMBQ) to give the corresponding allyl boronate esters. Transmetallation of an intermediate $\pi$-allylpalladium species with bispiacolatodiboron furnishes the desired allyl boronate, which is then trapped in situ with an aldehyde to give stereodefined homoallylic alcohols. 


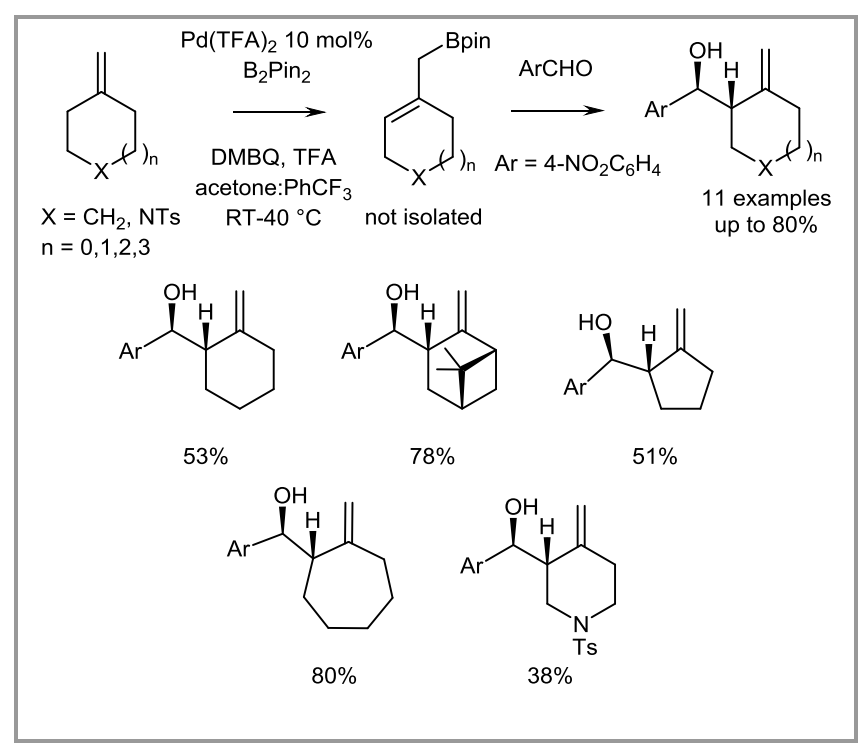

Scheme 33 Oxidative allylic borylation of exocyclic alkenes.

Extending the scope of this reaction to include activated terminal olefins, Gong has latterly reported a Brønsted acid-mediated allylboration of aldehydes and isatins using this chemistry (Scheme 34). ${ }^{57}$ The diphenyl phosphate additive plays a dual role in first facilitating allylic $\mathrm{C}-\mathrm{H}$ bond cleavage and then catalysing the allylboration by activating the carbonyl to nucleophilic attack. $N$-Fluorobenzenesulfonimide (NFSI) was used as a stoichiometric oxidant, and the resulting homoallylic alcohols were obtained in good yield with excellent diastereoselectivity for the antiproducts.

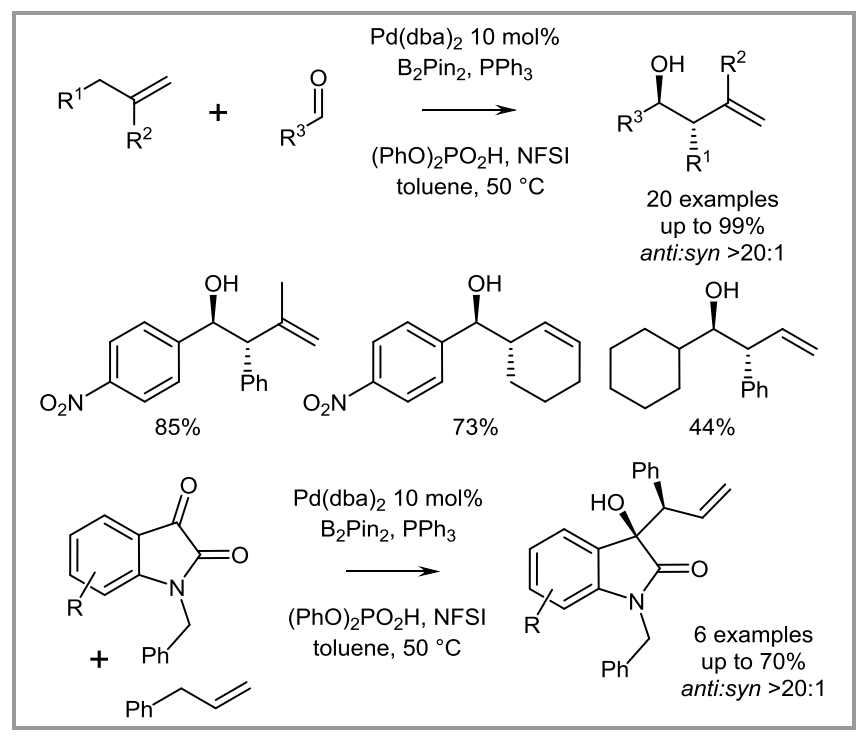

Scheme 34 Allylic borylation and in situ trapping to form stereodefined homoallylic alcohols.

Szabó has also reported a similar procedure for the oxidative allylic silylation of alkenes (Scheme 35), ${ }^{58}$ which is thought to proceed via a $\mathrm{Pd}(\mathrm{IV})$ mechanism due to the strong oxidants employed.

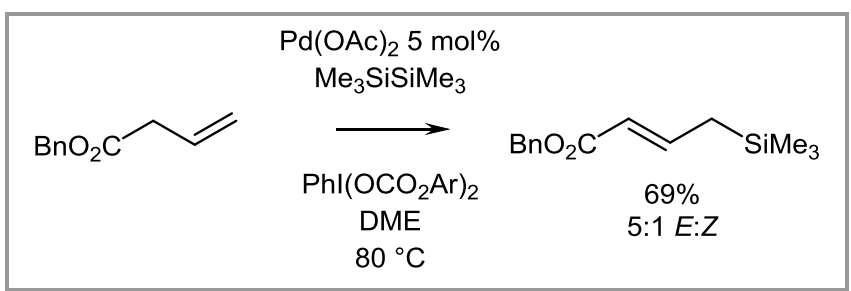

Scheme 35 Allylic silylation of terminal alkenes.

\section{The Wacker Oxidation}

\subsection{Background}

The development of an effective procedure for oxidizing readily available ethylene to acetaldehyde using a palladium/copper catalyst system was a landmark achievement, marking arguably the first ever industrial process employing a transition-metal catalyst. ${ }^{1}$ The reaction employs a $\mathrm{Pd}(\mathrm{II})$ salt as catalyst, with a copper co-catalyst and molecular oxygen as stoichiometric re-oxidant. The mechanism of the Wacker oxidation is far from trivial, and a detailed discussion of the mechanistic studies carried out has been the sole focus of a recent review aricle, ${ }^{3}$ so will not be covered here in detail. It is generally agreed, however, that the key step for the oxidation process involves an alkene hydroxypalladation, followed by $\beta$-hydride elimination and then $\mathrm{Pd}$ mediated isomerisation of the resulting enol to the aldehyde (Scheme 36). The mechanism of the hydroxypalladation step itself has been debated extensively, and convincing evidence for both syn and anti palladation mechanisms has been observed under different reaction conditions. ${ }^{2,3}$ It is likely that both pathways can operate, depending on the conditions of the reaction, but a cis-hydroxypalladation is thought to be more likely under conditions similar to the industrial process (low chloride concentration). ${ }^{2}$ However, a recent computational study has cast doubt on whether the Pd complex necessary for a cishydroxypalladation process can be generated under the reaction conditions. ${ }^{59}$ The complicated nature of the nucleopalladation step and the associated mechanistic studies have many implications for the development of new $\mathrm{Pd}(\mathrm{II})$-catalysed alkene oxidation reactions, particularly with regard to the stereochemical outcome. The role of the copper cocatalyst in the reaction is also controversial, and although its main role is probably in the re-oxidation of the palladium catalyst, its involvement in the alkene oxidation reaction itself has not been excluded and $\mathrm{Pd}-\mathrm{Cu}$ bimetallic species are often invoked. As will be seen in the following section, however, the Wacker oxidation can often be performed under copper-free conditions so such species are certainly not essential for the oxidation process to proceed efficiently. 


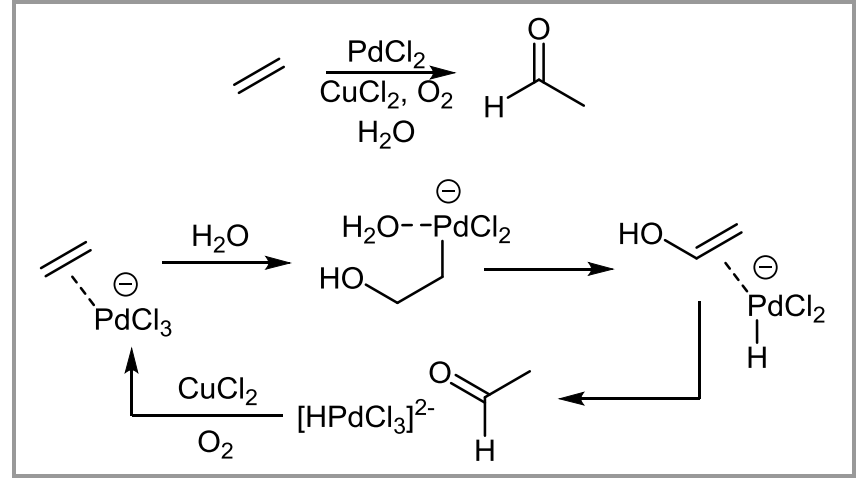

Scheme 36 A simplified mechanism for the Wacker oxidation of ethylene to acetaldehyde.

Through the use of a dipolar aprotic organic cosolvent (often DMF), the Wacker oxidation became a useful general method for the oxidation of terminal alkenes to methyl ketones which has been widely adopted in synthetic chemistry. ${ }^{60}$

In the majority of cases, the oxidation of a terminal alkene is highly regioselective, and leads exclusively to the formation of the methyl ketone. This is generally rationalized by enhanced stabilization of the developing cationic centre during the oxypalladation step at the more substituted carbon atom (Markovnikov's rule), in combination with a preference for the large palladium atom to bind to the less hindered carbon atom. When potential ligands for the palladium are present in the substrate, however, this regioselectivity can be reversed, with aldehydes being observed as significant byproducts or even as the sole oxidation product in some cases. ${ }^{61}$

Efficient catalytic turnover in laboratory Wacker oxidations frequently requires a high loading of copper salt and stoichiometric quantities are often used (Scheme 37). Oxidation of even simple alkenes such as 1-decene is typically carried out with 10 mol\% palladium catalyst and 1 equivalent of copper salt in order for efficient oxidation (a). ${ }^{60}$ For oxidation of more complex functionalised substrates, ${ }^{62}$ even larger quantities of the palladium and copper salts are often required $(\mathbf{b})$.

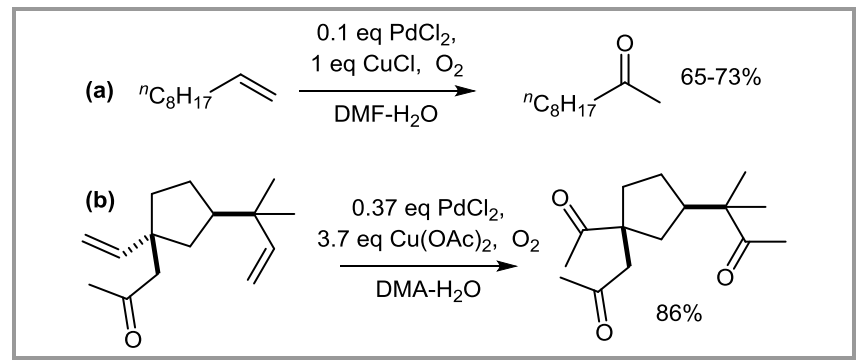

Scheme 37 Applications of the Wacker oxidation under 'traditional' reaction conditions.

More recent developments in this area have focused on the identification of new catalyst systems that enable efficient catalytic turnover of the palladium catalyst without the need for a copper co-catalyst. The scope of the reaction has also been effectively extended to include efficient oxidation of internal alkenes to ketones. In the following section, we will provide a summary of important new improvements to the Wacker oxidation which considerably widen the scope of this important transformation. We will also illustrate the extension of this chemistry to other related reactions which enable the formation of carbon-nitrogen bonds via an analogous mechanism. ${ }^{63}$

\subsection{Variation of the Co-Oxidant}

As outlined above, efficient catalytic turnover with molecular oxygen can be difficult to achieve in the Wacker oxidation and this often leads to the need for a large amount of copper co-oxidant. The use of stoichiometric amounts of copper salts is extremely undesirable as it leads to the production of large quantities of waste products, which considerably complicate isolation of the ketone product. As a consequence, there has been a lot of interest in the identification of cleaner co-oxidants that can lead to more efficient turnover, easier work-up and improved purification of the reaction mixture. The use of tertbutyl hydroperoxide as a co-oxidant has been particularly successful in this regard, as it is low-cost and readily available. In addition, the resulting byproducts ( ${ }^{t} \mathrm{BuOH}$ and water) are easily separable from the reaction products. Sigman and co-workers reported the use of ${ }^{t} \mathrm{BuOOH}$ in combination with an in situ generated cationic palladium species for the Wacker oxidation of styrenes (Scheme 38), ${ }^{64}$ which are often poor substrates under traditional Wacker oxidation conditions. The procedure is mild, and good yields were obtained from a selection of substrates (a). Notably, the catalytic system does not require the use of a dipolar aprotic solvent, presumably because the NHC ligand is able to stabilize the reactive $\mathrm{Pd}$ intermediates and prevent catalyst decomposition to produce palladium black. In later work (b), a modified procedure using a Quinox ligand in $\mathrm{CH}_{2} \mathrm{Cl}_{2}$ was successfully applied to the Wacker oxidation of a range of functionalised terminal alkenes. ${ }^{65}$ Notably, this included allylic alcohol derivatives which are well known to be difficult substrates for the Wacker oxidation, often leading to mixtures of regioisomeric products in low yield.

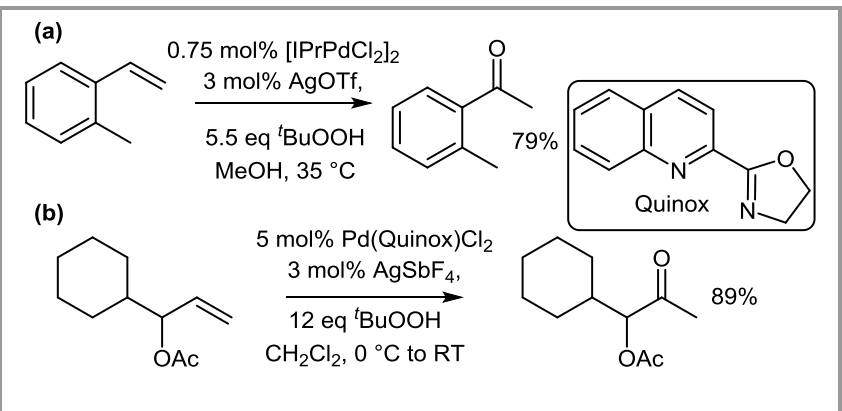

Scheme 38 Wacker oxidations with TBHP as a co-oxidant.

The reaction can be carried out under essentially anhydrous conditions in a non-polar organic solvent, 
and the ${ }^{t} \mathrm{BuOOH}$ is proposed to be directly involved in the reaction mechanism, acting as the nucleophile in the initial oxypalladation step (Scheme 39). ${ }^{66}$ This is proposed to lead to a cyclic intermediate which then collapses via a 1,2-hydride shift and cleavage of the oxygen-oxygen bond to give the ketone directly without the formation of an enol intermediate. Isotopic labeling studies supported the fact that an internal hydride shift was taking place (no exchange with the solvent), and that ${ }^{t} \mathrm{BuOOH}$ was the source of the ketone oxygen atom in the product. ${ }^{64}$

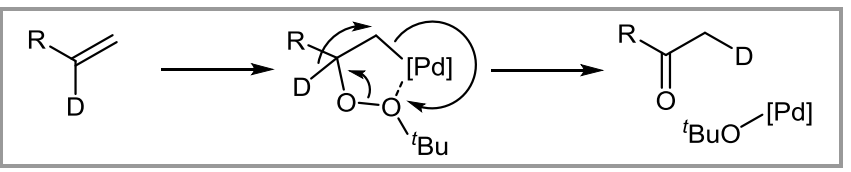

Scheme 39 Proposed mechanism for Wacker oxidation employing ${ }^{t} \mathrm{BuOOH}$ as a co-oxidant.

Very recently, the use of a simple iron salt as an effective co-oxidant for the Wacker oxidation has been reported. With 1.5 eq of $\mathrm{Fe}_{2}\left(\mathrm{SO}_{3}\right)_{4}$, efficient oxidation of a range of terminal alkenes to methyl ketones was possible in an acetonitrile-water solvent system (Scheme 40). ${ }^{67}$ The use of $\mathrm{CrO}_{3}$ as a stoichiometric co-oxidant under similar conditions has also been described by the same group. ${ }^{68}$

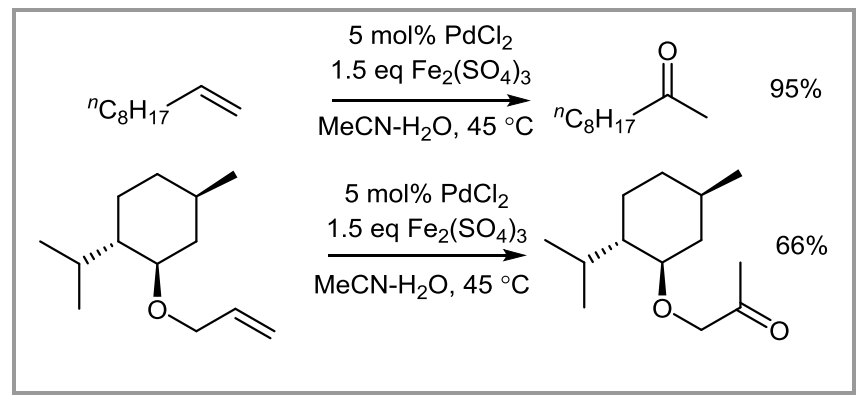

Scheme 40 Wacker oxidations with iron(III) sulfate as a cooxidant.

\subsection{Direct Oxygen-Coupled Wacker Oxidations}

Two reports in 2006 both described the development of a direct oxygen-coupled palladium-catalysed Wacker oxidation in a solvent mixture of dimethyl acetamide (DMA) and water. ${ }^{27,69}$ In the first report, ${ }^{27}$ a catalytic quantity of $\mathrm{PdCl}_{2} \quad(0.5-1 \mathrm{~mol} \%)$ was employed in the presence of an oxygen atmosphere to enable efficient oxidation of a range of terminal alkenes to methyl ketones (Scheme 41). The scope of the reaction included some functionalised compounds (alcohol, nitrile, ketone, phenol) as well as the double oxidation of octa-1,7-diene to the corresponding diketone. It should also be noted that closely related conditions were successfully applied to the oxidation of terminal alkenes to terminal allylic acetates (vide supra, Scheme 15). ${ }^{27}$ The main drawback of this method for laboratory use, however, is the requirement for a high pressure oxygen atmosphere. An important advantage, on the other hand, was that the catalyst could easily be recycled by simple extraction of the oxidation products with heptane, and re-use of the DMA- $\mathrm{H}_{2} \mathrm{O}$ layer in a subsequent reaction.

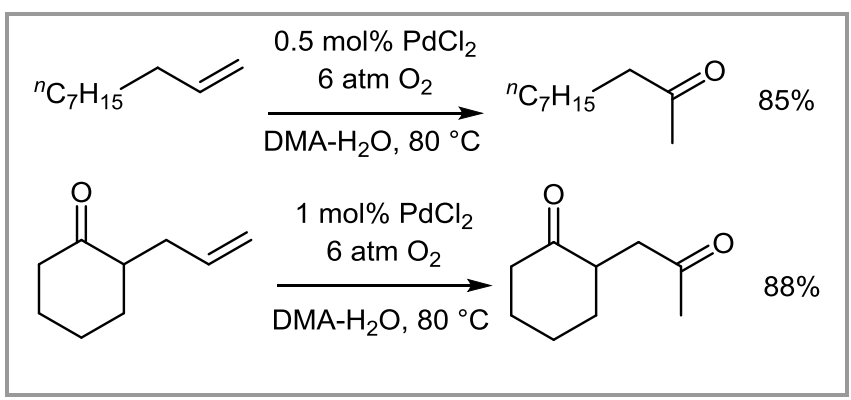

Scheme 41 Direct oxygen-coupled Wacker oxidation, catalysed by $\mathrm{PdCl}_{2}$.

Soon afterwards, a second report described the use of sparteine as a ligand for $\mathrm{PdCl}_{2}$ which enabled a direct oxygen coupled Wacker oxidation to take place at slightly elevated temperature under an atmosphere of oxygen provided by a balloon (Scheme 42 ). ${ }^{69}$

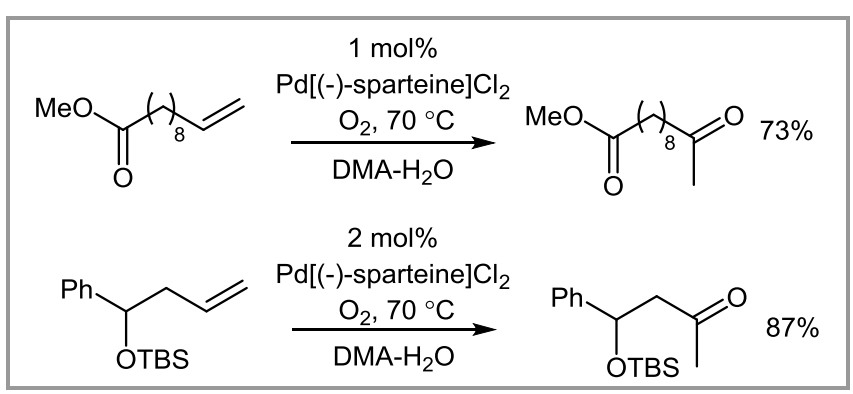

Scheme 42 Direct oxygen-coupled Wacker oxidation, catalysed by $\operatorname{Pd}[(-)$-sparteine $] \mathrm{Cl}_{2}$.

Again, the reaction was tolerant of a variety of functional groups (alcohol, ester, acetal, ether, etc) and the oxidation of alkenes bearing nearby chiral centres was shown to proceed without significant racemisation.

In a recent report, a direct oxygen-coupled Wacker oxidation was reported using a simple palladium(II) salt in the presence of 1 equivalent of trifluoroacetic acid (Scheme 43). ${ }^{70}$ Although elevated temperatures were required, the reaction works without the need for complex ligands. Interestingly, it was found that the process could be adapted to the direct conversion of terminal alkenes to $\alpha, \beta$-unsaturated ketones via a tandem oxidation/dehydrogenation process. Although most of the examples of this reaction involved dehydrogenation to form highly conjugated systems, the process could be adapted to less activated substrates by reducing the TFA loading and addition of a catalytic quantity of piperidine. 


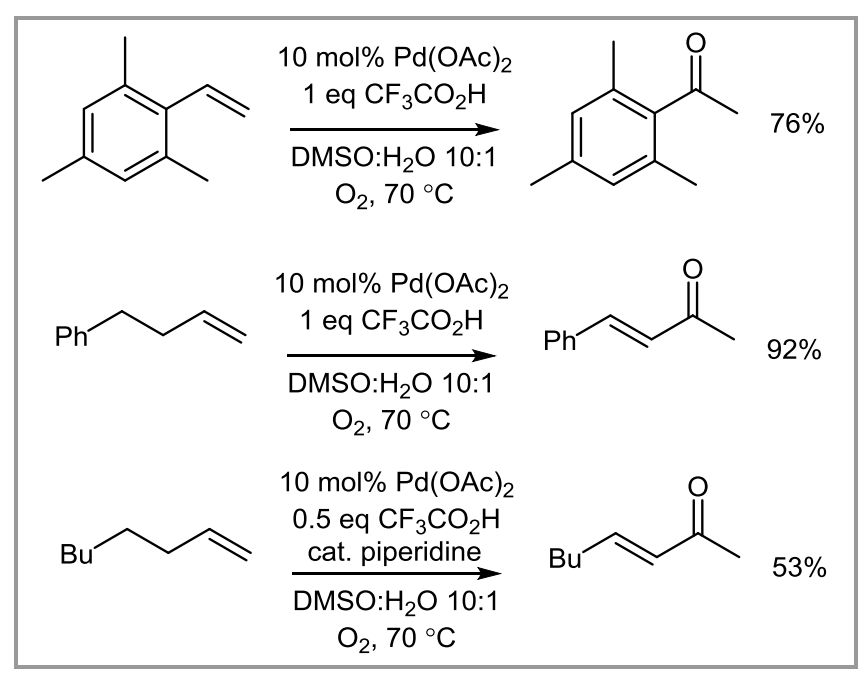

Scheme 43 Direct oxygen-coupled Wacker oxidation and tandem Wacker oxidation/dehydrogenation in the presence of trifluoroacetic acid.

\subsection{Aldehyde-Selective Wacker Oxidations}

As described above, the Wacker oxidation of terminal alkenes typically leads to the formation of methyl ketones with high regioselectivity, unless the substrate contains coordinating groups that can direct the palladium towards the internal carbon atom. ${ }^{61}$ The oxidation of alkenes containing nearby oxygen atoms, such as the acetonide shown below (Scheme 44), ${ }^{71}$ often leads to regioselective aldehyde formation. This may be a consequence of coordination of the acetonide to the palladium favoring anti-Markovnikov oxypalladation. Interestingly, oxidation of the corresponding diol obtained after acetonide removal led to formation of the methyl ketone with high regioselectivity.

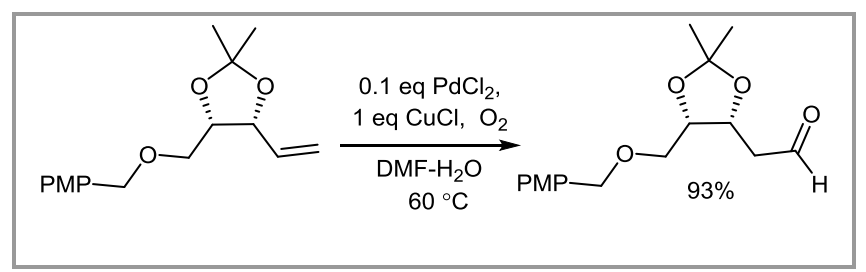

Scheme 44 Substrate-directed Wacker oxidation of a terminal alkene to an aldehyde.

However, in recent years considerable progress has been made on the identification of reaction conditions and/or catalysts that inherently favor the formation of aldehydes, even from unfunctionalised terminal alkenes. This aspect of the Wacker oxidation has been recently reviewed, ${ }^{72}$ so only a brief summary of recent developments will be provided here.

A useful approach to achieving anti-Markovnikov selectivity is to employ a bulky alcohol as the oxygen nucleophile (Scheme 45) which leads to preferential attack at the less hindered end of the alkene and, after $\beta$-hydride elimination from the $\sigma$-organopalladium intermediate, the enol ether of the corresponding aldehyde.

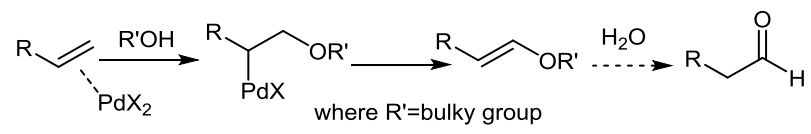

Scheme 45 anti-Markovnikov selectivity in the Wacker oxidation through the use of a bulky alcohol nucleophile.

In a recent example, the efficient conversion of terminal alkenes to pinacol acetals of the corresponding aldehydes was reported in the presence of a simple commercially available palladium(II) catalyst and benzoquinone as a co-oxidant (Scheme 46). ${ }^{73}$

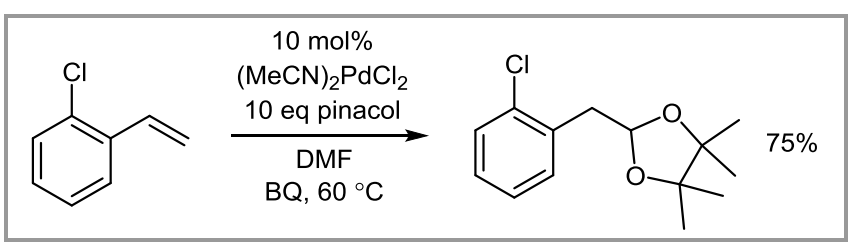

Scheme 46 Direct conversion of terminal alkenes to pinacol acetals of aldehydes under Pd-catalysed oxidative conditions.

In early reports, it was observed that the use of tertbutanol as solvent led to increased selectivity for the formation of aldehydes in the Wacker oxidation. ${ }^{74}$ This is likely to be due to the initial formation of a tert-butyl enol ether via the path shown in Scheme 45. Under the acidic conditions of the Wacker oxidation this could readily undergo hydrolysis to the corresponding aldehyde. However, these early reactions suffered from low conversions and poor catalytic turnover. Recently, though, conditions for the efficient oxidation of styrenes to the corresponding aldehydes in ${ }^{t} \mathrm{BuOH}$ have been developed (Scheme 47). ${ }^{75}$

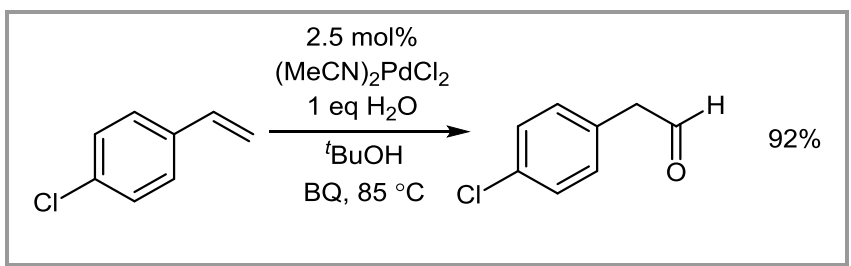

Scheme 47 anti-Markovnikov selectivity in the Wacker oxidation of styrenes through the use of a bulky alcohol nucleophile.

In a more recent report, related conditions were developed for the aldehyde-selective Wacker oxidation of allylic esters (Scheme 48). ${ }^{76}$ Importantly, it was demonstrated that the allylic esters undergo rapid isomerisation between the branched and linear isomers under the reaction conditions. As the branched isomer undergoes Wacker oxidation much more rapidly, this therefore allows the same $\beta$ acetoxyaldehyde oxidation product to be obtained starting from either isomer of the allylic ester (or even a mixture of the two). 


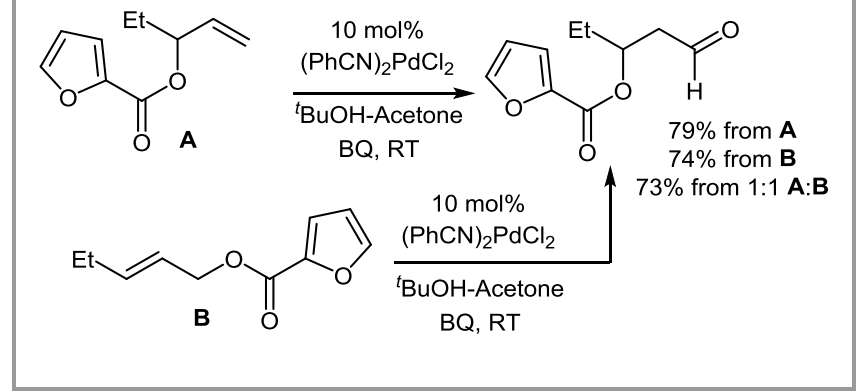

Scheme 48 anti-Markovnikov Wacker oxidation of allylic esters.

The chemistry was also extended to the regioselective oxidation of protected allylic amines (Scheme 49). ${ }^{77-78}$ This gave the corresponding aldehydes with exceptionally high regioselectivity (a). The process could also be combined with a Pd-catalysed [2,3]sigmatropic rearrangement of the corresponding primary allyl imidate (b). ${ }^{78}$ This reaction also provided an effective method for the asymmetric synthesis of $\beta$-aminoacids. ${ }^{77}$ It should be noted, however, that protected allylic amines and alcohols typically show a preference for the formation of aldehydes even under traditional Wacker oxidation conditions, though this oxidation procedure generally provides better conversions and also enhances the inherent regioselectivity preferences of the substrates. Mechanistic studies suggested that the reaction did not proceed via simple hydrolysis of a tert-butyl enol ether as shown in Scheme 45, as no deuterium was incorporated into the substrate when fully labeled ${ }^{t} \mathrm{BuOD}$ was used. A mechanism involving a Pdmediated cleavage of the enol ether was proposed leading to direct formation of isobutylene. ${ }^{78}$

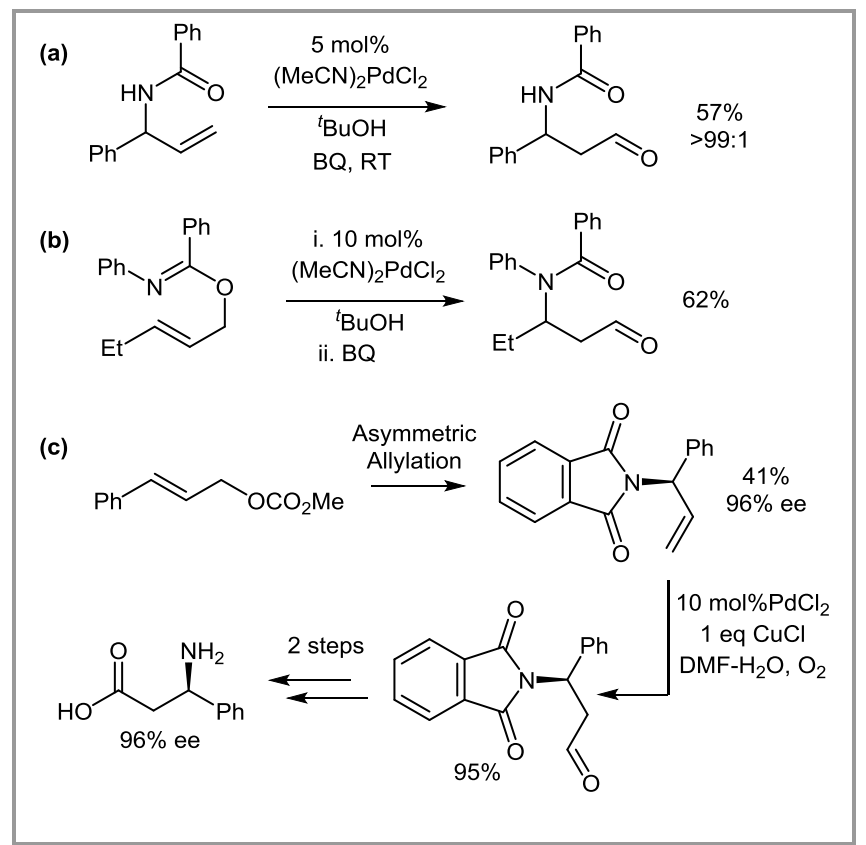

Scheme 49 Oxidation of protected allylic amines to aldehydes and its application to the synthesis of $\beta$-amino acids.

Although the use of ${ }^{t} \mathrm{BuOH}$ as solvent has proved useful for obtaining aldehyde-selective oxidations in the above cases, it is not generally applicable to all alkene substrates. Moreover, the use of stoichiometric co-oxidants is required and oxygen cannot as yet be effectively employed as the terminal oxidant. However, the use of a nitrite-containing oxidation system $^{79}$ has been shown to provide enhanced aldehyde-selectivity in the Wacker oxidation of a wider range of functionalised ${ }^{80}$ and unfunctionalised alkenes (Scheme 50). ${ }^{81}$ Furthermore, this system, whilst relatively complex, is able to function effectively with $\mathrm{O}_{2}$ as the stoichiometric oxidant. For example, 8-bromooct-1-ene was oxidized to the corresponding aldehyde in $65 \%$ yield (a), and a TBS ether was oxidized to the corresponding aldehyde in $71 \%$ yield on a $4 \mathrm{~g}$ scale $(\mathbf{b})$.

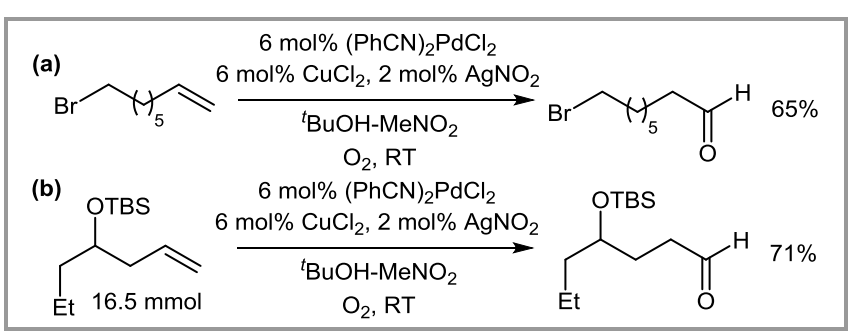

Scheme 50 Aldehyde-selective Wacker oxidation using a nitrite co-oxidant system.

These conditions were demonstrated to promote aldehyde-selective oxidations irrespective of the inherent preference of the substrate. Labeling studies with ${ }^{18} \mathrm{O}$ suggested that the oxygen in the aldehyde was derived from the nitrite co-oxidant. ${ }^{81}$ A radical addition mechanism has been suggested to account for the unusual regioselectivity, though control experiments suggested that the $\mathrm{Pd}$ catalyst was responsible for mediating the addition of nitrite to the alkene and not the co-oxidants present in the system. A very recent computational study of the mechanism of this reaction suggested that the product-determining step was in fact an intramolecular 1,2-hydride shift and not the initial oxypalladation step. ${ }^{82}$

\subsection{Wacker Oxidation of Internal Alkenes}

Under typical Wacker oxidation conditions, internal alkenes generally show low reactivity unless there is a strongly activating group present. In general this has meant that Wacker oxidations of internal alkenes were not synthetically practical. This is now no longer the case, as considerable progress has been made in the past few years on the development of catalyst systems that are able to efficiently oxidise internal alkenes to the corresponding ketones.

Kaneda and co-workers were able to adapt the conditions for their direct oxygen-coupled Wacker oxidation (Scheme 41) to the conversion of internal alkenes to ketones (Scheme 51) ${ }^{83}$ As before, the use of a mixed solvent system containing DMA- $\mathrm{H}_{2} \mathrm{O}$ was found to be essential for efficient catalytic turnover. Interestingly, attempted oxidations using $\mathrm{CuCl}_{2}$ as cooxidant in the same solvent system led to considerably lower conversions with the direct oxygen-coupled reaction giving significantly higher yields. As before, 
the main drawback of this method is the requirement for a higher pressure oxygen atmosphere.

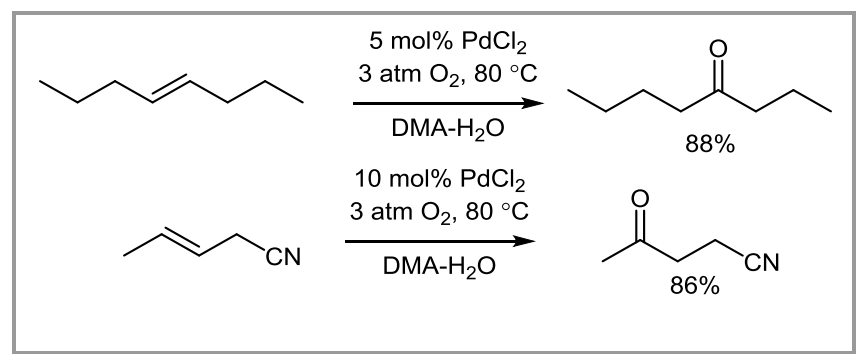

Scheme 51 Wacker oxidation of internal alkenes using a $\mathrm{PdCl}_{2} / \mathrm{O}_{2}$ system.

Subsequently, Grubbs and co-workers demonstrated that a cationic palladium(II) salt was highly effective for oxidation of internal alkenes (Scheme 52) ${ }^{84}$ This was generated in situ from $\mathrm{Pd}(\mathrm{OAc})_{2}$ and $\mathrm{HBF}_{4}$, with the Brønsted acid also accelerating the oxidation reaction as even reactions using a pre-formed cationic complex, $(\mathrm{MeCN})_{4} \mathrm{Pd}\left(\mathrm{BF}_{4}\right)_{2}$, were accelerated by addition of $\mathrm{HBF}_{4}$. Again, the solvent system was found to be crucial with a mixture of DMA-MeCN$\mathrm{H}_{2} \mathrm{O}$ providing the best option. It should be noted that the presence of DMA was demonstrated to prevent isomerisation of the internal alkene prior to oxidation, whereas $\mathrm{MeCN}$ was observed to accelerate the oxidation itself. Benzoquinone (BQ) was employed as a convenient co-oxidant.

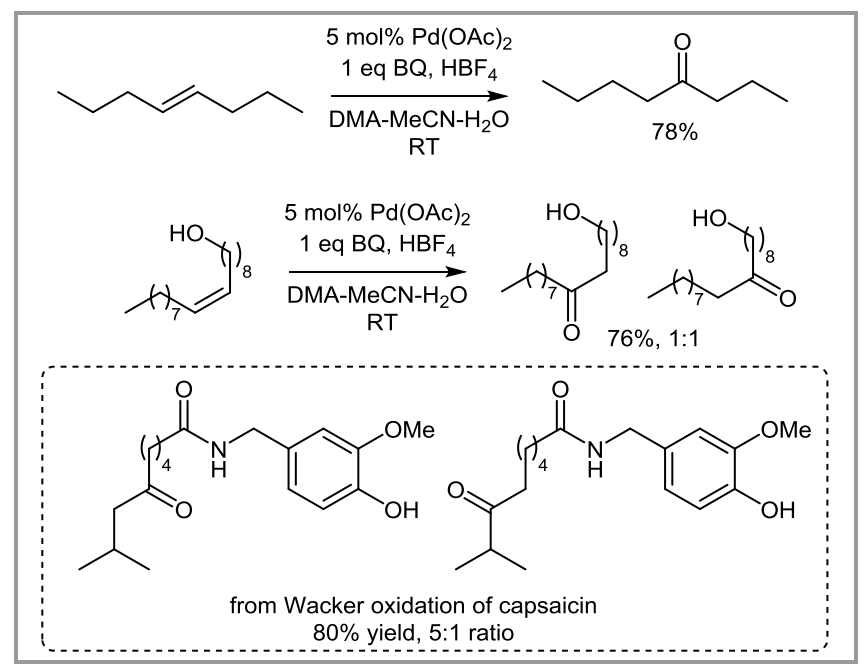

Scheme 52 Wacker oxidation of internal alkenes with benzoquinone $(\mathrm{BQ})$ as the co-oxidant in the presence of a strong Brønsted acid.

Furthermore, it was shown that the reaction could be coupled to molecular oxygen as the terminal oxidant (Scheme 53) using Bäckvall's BQ/Fe(pc) catalyst system (where $\mathrm{pc}=$ phthalocyanine) ${ }^{85}$

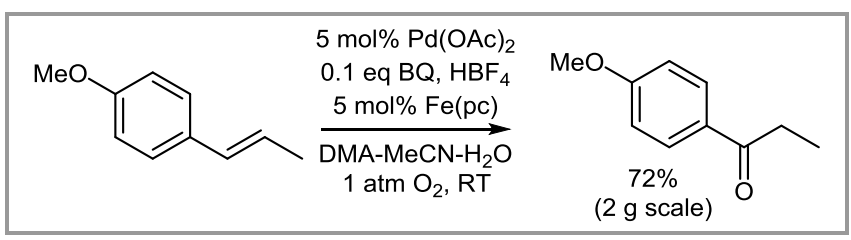

Scheme 53 Brønsted acid assisted Wacker oxidation of internal alkenes using molecular oxygen as the stoichiometric oxidant.

The reaction was subsequently extended to more functionalised terminal alkenes and the directing effect of a range of groups evaluated (Scheme 54). ${ }^{86}$ Usefully, electron-withdrawing groups such as esters or ethers were shown to favour formation of the distal ketone. In the case of these functionalised alkenes, double bond isomerisation was not found to be a significant problem. In fact, addition of DMA to the solvent system was found to be very detrimental to the rate of oxidation and overall conversion.

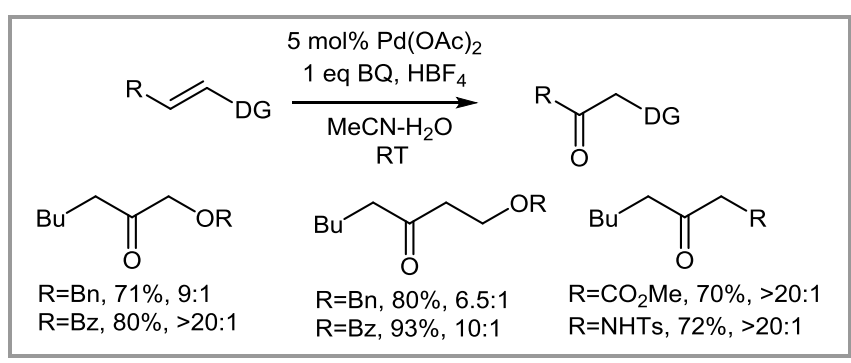

Scheme 54 Wacker oxidation of internal alkenes bearing directing functional groups.

From a detailed substrate study and several competition experiments, the directing group influence was suggested to be largely down to electronic effects rather than chelation or direct nucleophilic participation in the reaction. This was further supported by a subsequent paper in which the presence of an allylic or homoallylic trifluoromethyl group was found to exert a powerful directing effect on the regioselectivity of the Wacker oxidation (Scheme 55). ${ }^{87}$ Notably, the $\mathrm{CF}_{3}$ group exerted a stronger directing effect than groups examined in the earlier study such as esters or ethers. Unfortunately, the oxidation of alkenes with a $\mathrm{CF}_{3}$ substituent attached directly to the alkene was not possible under these conditions.

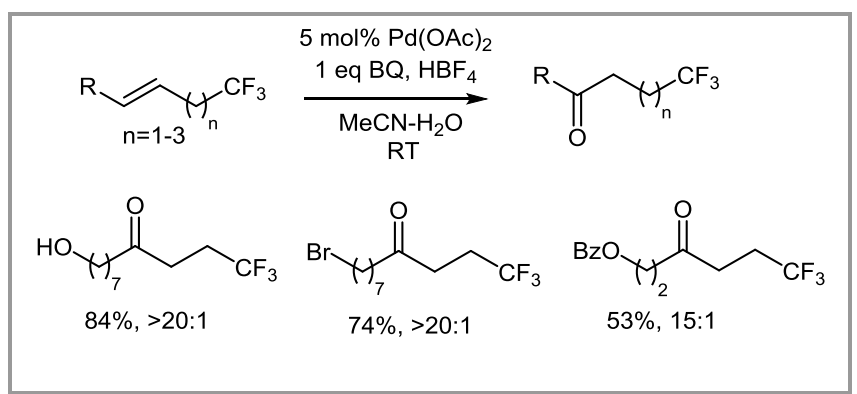

Scheme 55 Wacker oxidation of $\mathrm{CF}_{3}$-containing internal alkenes.

The use of strong acid in the above reactions is likely to preclude efficient oxidation of substrates containing acid-sensitive protecting groups. Sigman and co- 
workers reported that their catalyst system for Wacker oxidation, based upon a Quinox-ligated palladium complex and using ${ }^{t} \mathrm{BuOOH}$ as a co-oxidant, could be applied to internal alkenes (Scheme 56). ${ }^{88}$ Again, this system probably involves a cationic palladium species which is generated in situ. Interestingly, the presence of water in the aqueous solution of ${ }^{t} \mathrm{BuOOH}$ was thought to inhibit alkene isomerisation in this case.

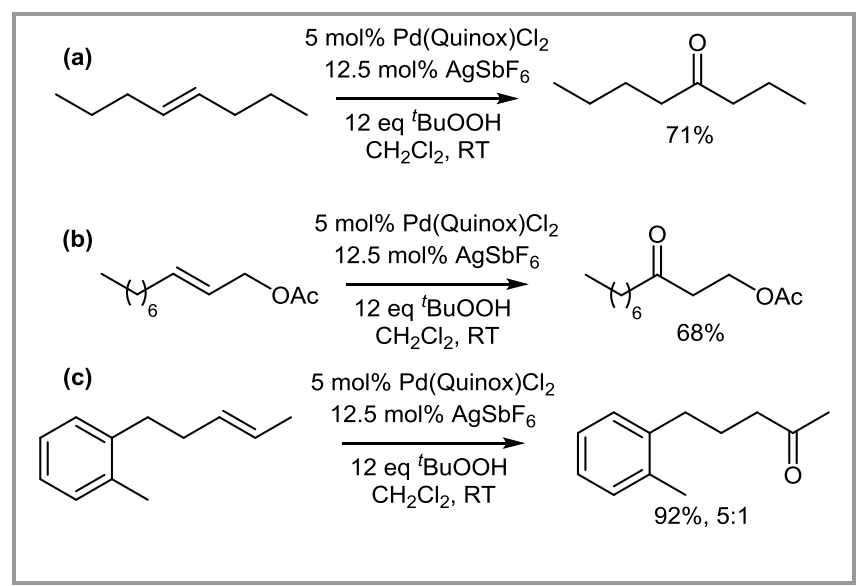

Scheme 56 Wacker oxidation of internal alkenes using a Quinoxligated palladium catalyst with tert-butyl hydroperoxide as the stoichiometric oxidant.

Again, the regioselectivity of oxidation appears to be largely driven by electronic effects as this ligated palladium species is unlikely to co-ordinate to groups in the substrate during the reaction. Symmetrical internal alkenes gave single products (a), and nonsymmetrical unfunctionalised alkenes gave the expected mixture of isomeric ketones. An allylic ester underwent selective oxidation at the distal carbon (b). Interestingly, however, reasonable selectivity was observed in the oxidation of a largely unfunctionalised arene-containing alkene (c).

\subsection{Aza-Wacker Oxidations}

In the presence of a suitable nitrogen nucleophile, the oxidation of alkenes with a Pd(II) catalyst can be used to generate enamines in the nitrogen analogue of the Wacker oxidation. This 'aza-Wacker' oxidation has largely been restricted to intermolecular cyclisation reactions, which can often be usefully harnessed in the synthesis of medicinally relevant heterocycles. ${ }^{89}$ However, more recently a number of intermolecular reactions have been reported.

The direct conversion of acrylates and other electrondeficient alkenes to $Z$-enamines by $\mathrm{Pd}$-catalysed reaction with anilines was recently reported (Scheme 57). ${ }^{90}$ The catalyst system employs $\mathrm{Pd}(\mathrm{OAc})_{2}$ in the presence of excess $\mathrm{LiBr}$ and uses oxygen as the stoichiometric oxidant (a). The authors speculated that the role of $\mathrm{LiBr}$ was as a stabilizing ligand for the active palladium catalyst that prevented coordination of the aniline and subsequent precipitation/decomposition of the palladium from the reaction mixture. Under slightly modified conditions with the presence of $\mathrm{H}_{2} \mathrm{O}_{2}$ as an additional oxidising reagent, the $\mathrm{LiBr}$ serves as a bromine source and the ${ }_{92}$ corresponding brominated alkenes are obtained (b). ${ }^{91-}$

$$
\text { (b) }
$$

Scheme 57 Aza-Wacker reactions of electron-deficient alkenes and anilines.

The Z-selectivity in the case of the non-brominating conditions was attributed to hydrogen-bonding interactions between the $\mathrm{NH}$ and the carbonyl. ${ }^{90}$ The $Z$-enamines are useful precursors for the synthesis of a selection of nitrogen heterocycles (Scheme 58). For example, $\mathrm{Pd} / \mathrm{Cu}$-mediated cyclisation of an $\mathrm{N}$-aryl enamine gave an indole (a) or $\mathrm{Cu}$-mediated reaction with benzonitrile gave a pyrazole (b). Routes to pyrroles and dihydropyridines were also developed.

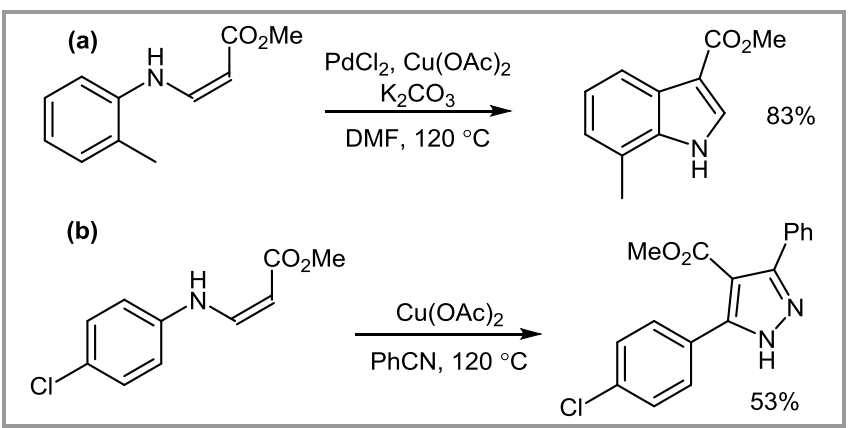

Scheme 58 Conversion of $Z$-enamines into nitrogen heterocycles

A closely related procedure for the synthesis of $Z$ enamines via an aza-Wacker oxidation was described by another research group who employed ortho-substituted anilines to prevent deactivation of the catalyst by the nitrogen nucleophile (Scheme 59). ${ }^{93}$ In this case, with $N$-methylpyrrolidinone (NMP) as solvent, they were able to use air as the stoichiometric oxidant. A very similar extension of this latter method to give $\mathrm{N}$-alkylanilines was reported very recently. ${ }^{94}$

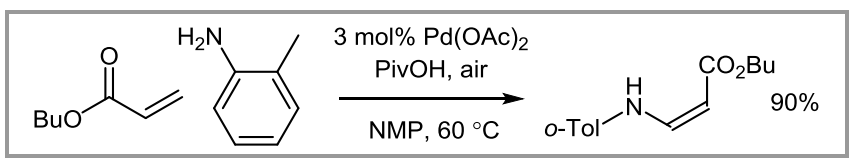

Scheme 59 Aza-Wacker reactions of electron-deficient alkenes and hindered anilines with air as the stoichiometric oxidant.

The $N$-vinylation of indoles has also been described under typical Wacker-type conditions in DME as solvent (Scheme 60). ${ }^{95}$ Styrenes underwent selective $\mathrm{C}-\mathrm{N}$ bond formation at the internal carbon of the alkene (a), whereas electron deficient alkenes or 
allylic ethers led to selective $\mathrm{C}-\mathrm{N}$ bond formation at the terminal carbon atom (b). With some cyclic alkenes, enamines were not formed as the major product, with alkene isomerisation taking place under the reaction conditions.

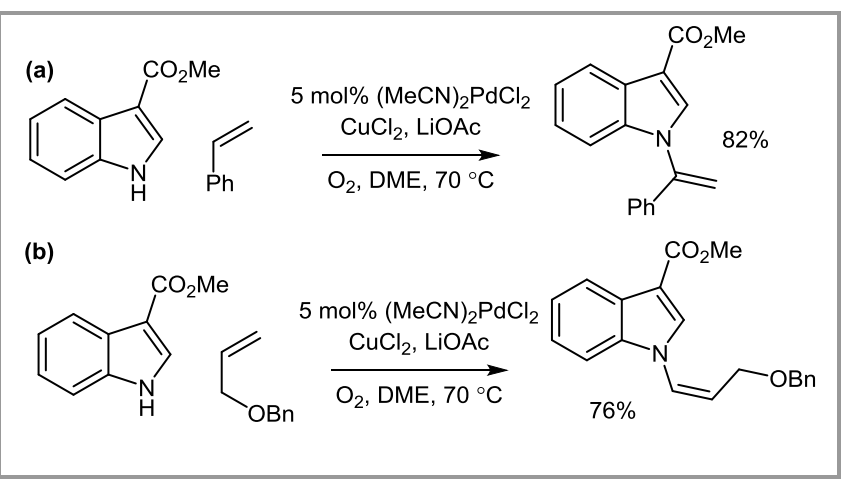

Scheme 60 Palladium-catalysed $N$-vinylation of indoles.

\section{Intermolecular 1,2-Difunctionalisation of Alkenes}

\subsection{Introduction}

Metal catalysed vicinal difunctionalisation of olefins represents an important tool in organic chemistry to afford valuable saturated intermediates for synthesis. From a fundamental point of view, this process results in the formation of two new C-X bonds in a single operation. The Sharpless dihydroxylation made a huge impact in this research area ${ }^{96}$ and this transformation has been extremely well studied, allowing effective preparation of chiral 1,2-diols from a very wide range of alkenes. In the past 20 years, palladium catalysis has begun to emerge as a new strategy for the 1,2-difunctionalisation of alkenes, enabling the regioselective formation of two new bonds in a single reaction. ${ }^{97}$

Palladium catalysed 1,2-difunctionalisation reactions of alkenes occur via two separate steps. The first stage of the transformation is a nucleopalladation process where an alkene, activated beforehand with an electrophilic palladium catalyst, is attacked by a nucleophile resulting in the formation of a Pd-carbon $\sigma$-bond and a new C-Nu bond (Scheme 61). This step is the same as the first step of the Wacker oxidation and can take place with syn or anti selectivity. Mechanistic studies carried out to rationalise the stereochemistry of nucelopalladation underline the fact that the outcome of the reaction is highly dependent on the nature of the substrate, catalyst and/or reaction conditions. A detailed summary of all the effort provided in this research area can be found in recent reviews., $35 \mathrm{~b}$ The second part of the transformation is a functionalisation stage where the $\sigma$-alkyl-Pd intermediate is trapped with a functional group to create a new C-FG bond. The overall 1,2difunctionalisation transformation results in the creation of two new bonds to the carbon atoms that originally formed the alkene.

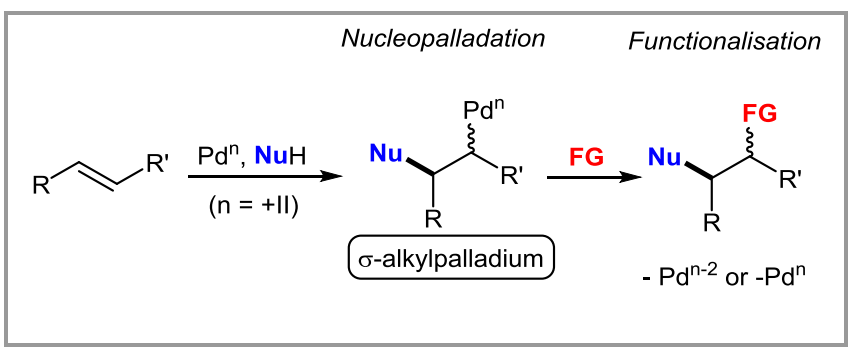

Scheme 61 Overview of Pd-catalysed 1,2-difunctionalisation of alkenes.

As previously described in section 3, the $\sigma$-alkyl palladium complex can easily undergo $\beta$-hydride elimination to afford the Wacker type product (Pathway A, Scheme 62). In contrast, functionalisation can occur preferentially if the Pdalkyl intermediate is either efficiently stabilised or if it undergoes a sufficiently rapid transformation that can out-compete $\beta$-hydride elimination. Several different mechanisms are possible for efficiently intercepting the Pd-alkyl complex. The palladium centre can be displaced by a nucleophile (Nu'-H or Nu'-metal) with or without prior coordination of the nucleophile to the palladium (Pathway B). The displacement step can be part of a $\mathrm{Pd}(\mathrm{II}) / \mathrm{Pd}(0)$ catalytic cycle with an oxidant being required to re-oxidise the $\operatorname{Pd}(0)$ species resulting from reductive elimination. Alternatively, addition of a nucleophile can also occur with a strong oxidant via a Pd(IV)-alkyl intermediate. This intermediate is particularly disposed to reductive elimination or to nucleophilic displacement, forming a second $\mathrm{C}-\mathrm{Nu}$ ' bond with concomitant regeneration of the $\mathrm{Pd}(\mathrm{II})$ active species. $^{98}$ In both cases these displacements can occur with either retention or inversion at the C-Pd bond. Another approach to intercept the Pd-alkyl complex consists of insertion of an olefin into the Pd-alkyl bond followed by a $\beta$ hydride elimination process to release the product and the $\mathrm{Pd}(\mathrm{II})$ catalyst (Pathway $\mathrm{C}$, a Heck reaction). Similarly, insertion of carbon monoxide into the Pdalkyl bond can occur and deliver carbonylated compounds such as esters (Pathway D). 


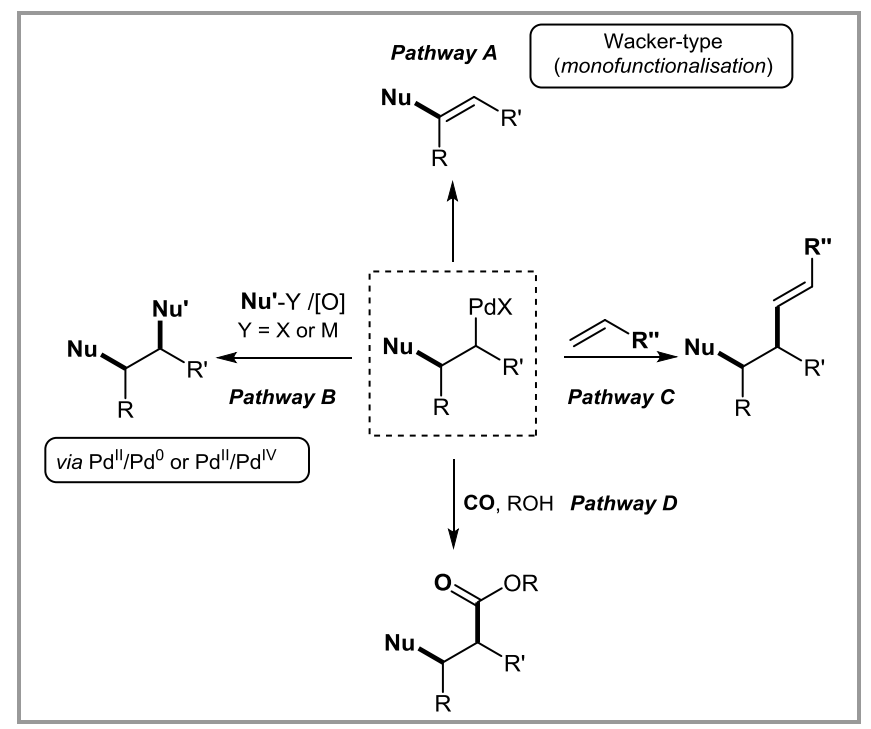

Scheme 62 Pathways for Pd(II)-catalysed 1,2-difunctionalisation reactions.

Over the past 20 years, many advances have been made in this research area and a number of innovative methodologies have been reported. 1,2Difunctionalisation of alkenes via intramolecular nucleopalladation has already been well covered in recent literature reviews and will not be discussed in detail here. ${ }^{97}$ This section of the review will therefore largely focus on processes involving an intermolecular oxypalladation or aminopalladation reaction (oxygenor nitrogen-based nucleophiles). Moreover, hydrofunctionalisation resulting from the trapping of the Pd-alkyl species by a proton and oxidative Heck coupling leading to the functionalisation of only one carbon of the alkene will also not be discussed.

\subsection{Oxyhalogenation Reactions}

The first report of a palladium catalysed intermolecular 1,2-difunctionalisation of an alkene was in 1970 by Stangl and Jira with the formation of chlorohydrin from ethylene in the presence of palladium dichloride catalyst and copper(II) chloride (Scheme 63). ${ }^{99}$ The chlorohydrin was only considered as a side product of the Wacker oxidation of ethylene to acetaldehyde and its formation was strongly correlated to the presence of copper chloride and free chloride in the reaction.

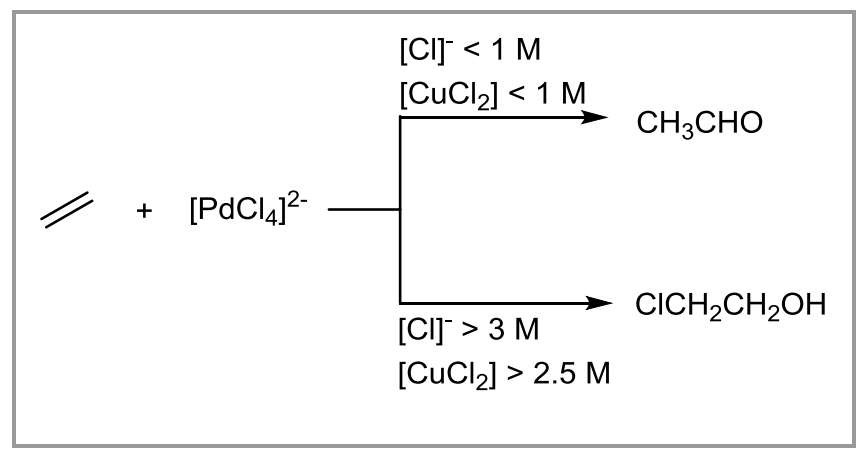

Scheme 63 Observation of chlorohydrin formation during the Wacker oxidation.
Following this original result, Bäckvall et al. (as part of a mechanistic study on the palladium catalysed Wacker oxidation of ethylene) postulated that chlorohydrin and acetaldehyde arise from the same $\beta$ hydroxypalladium species (Scheme 64). ${ }^{100}$ The chlorohydrin obtained was shown to result from synaddition of the hydroxyl group and the chloride. When the deuterated $E$-alkene was employed the threo product was observed. Previous studies by Bäckvall suggested that the palladium displacement by chloride ions occurs with inversion of configuration. ${ }^{101}$ Consequently, to account for the stereochemistry of the chlorohydrin product, the nucleopalladation was proposed to be an anti-process.

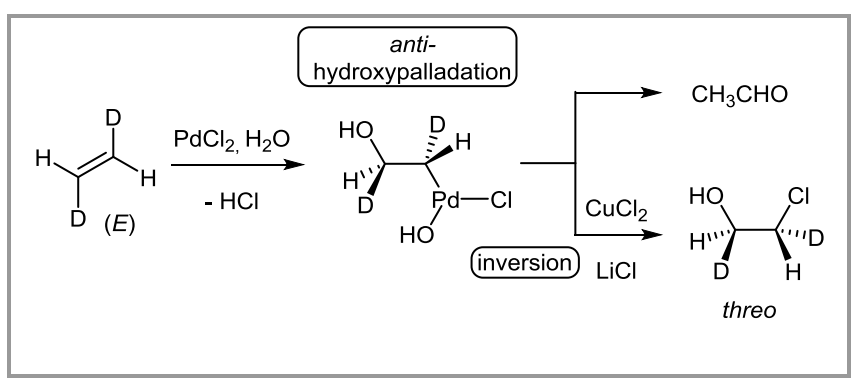

Scheme 64 Stereoselective formation of a chlorohydrin from selectively deuterated ethylene.

These two reports undoubtedly led the way for the development of 1,2-difunctionalisation reactions of olefins via palladium catalysis. However, it was not until 1995 that Henry et al. presented an asymmetric synthesis of chlorohydrins based on a palladium catalysed strategy (Scheme 65). The optimal catalytic system for this transformation involved a bimetallic palladium catalyst with a chiral bidentate phosphine (BINAP) in combination with a triketone ligand (1phenyl-1,3,5-hexatrione). ${ }^{102}$ Copper(II) chloride was used in large excess as the oxidant. It is interesting to note that when copper(II) bromide was employed as terminal oxidant the reaction afforded the corresponding 1,2-dibromoalkane. ${ }^{103}$ The system was applicable to a small range of 1-substituted propenyl ethers and moderate to high regio- and enantioselectivities were achieved. The author suggested that the rigidity of the catalyst induced by the coordination of the BINAP in a bridging fashion and the forced coordination of the alkene near the chiral ligand were the reasons behind the good asymmetric induction. Notably, a distinctive feature of this catalytic system is the use of a diphosphine ligand. Indeed, phosphine ligands are generally avoided in oxidative palladium chemistry, firstly because of their potential incompatibility with an oxidizing environment, but also due to their strong $\sigma$ donor character which decreases the electrophilicity of the palladium centre and disfavours the coordination of the olefin. Further studies to optimise the procedure found that high enantioselectivities could also be achieved with chelating chiral diamine and bisoxazoline ligands. 


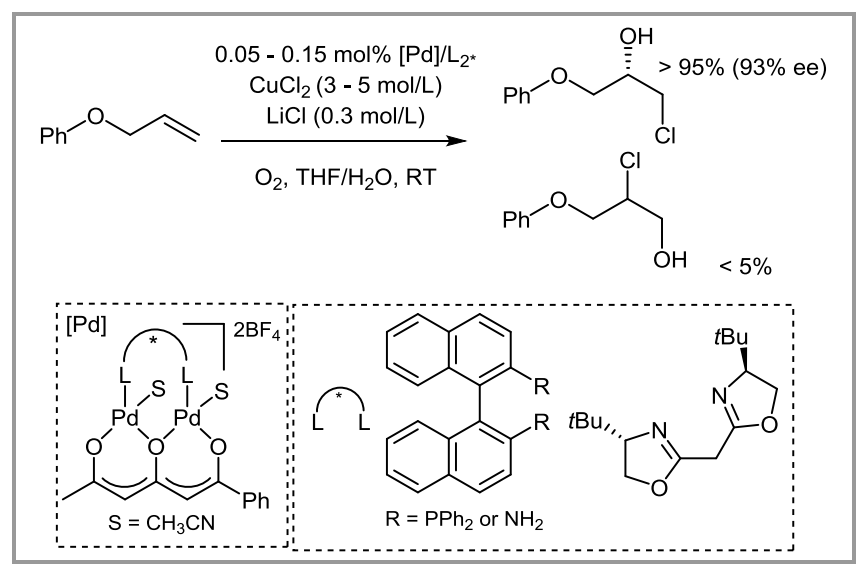

Scheme 65 Enantioselective chlorohydration of allyl ethers with a chiral palladium catalyst.

\subsection{Dioxygenation Reactions}

In 2005, Muzart, Le Bras and co-workers developed a palladium catalysed procedure for preparation of 2-(1,2-dialkoxypropyl)phenols starting from 2-allylphenols (Scheme 66). ${ }^{104}$ Palladium(II) trifluoroacetate was used as catalyst in combination with a hydrophilic diamine $\left(\mathrm{L}_{\mathrm{H}}\right)$. The reaction was performed in a mixture of water:methanol $(1: 1)$ at 50 ${ }^{\circ} \mathrm{C}$ and aqueous hydrogen peroxide played the role of oxidant. Under these conditions, conversions were quite high, although the 1,2-diol product was obtained along with the monomethylated compound with no significant diastereoselectivity. Unfortunately, in water alone the 1,2-diol was obtained in only $50 \%$ yield.

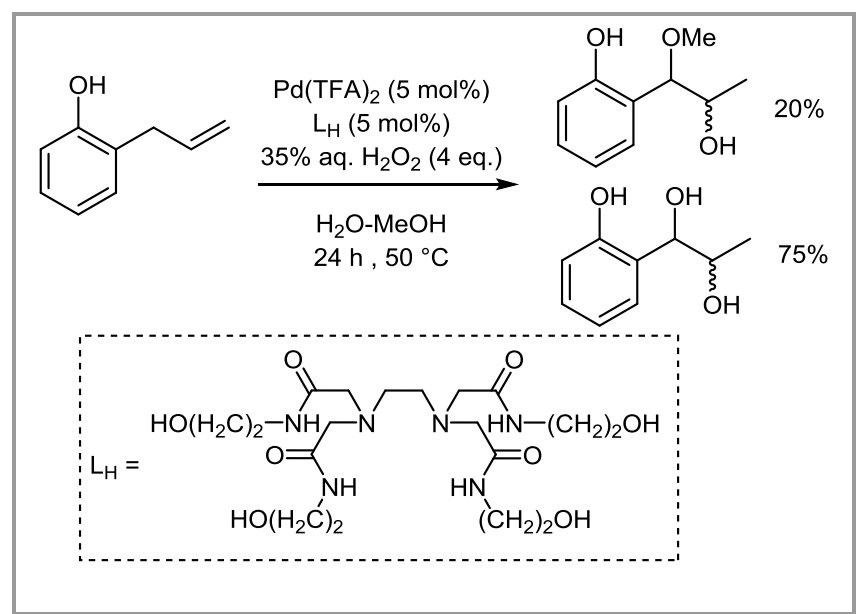

Scheme 66 Palladium-catalysed dihydroxylation of 2-allylphenols.

The mechanism of this transformation was studied further and was believed to involve three steps (Scheme 67). ${ }^{105}$ A palladium catalysed alkene isomerisation proceeds, followed by Pd-catalysed epoxidation of the internal alkene with hydrogen peroxide. Finally, the epoxide undergoes ring opening in the presence of the Lewis acidic catalyst to afford the products.

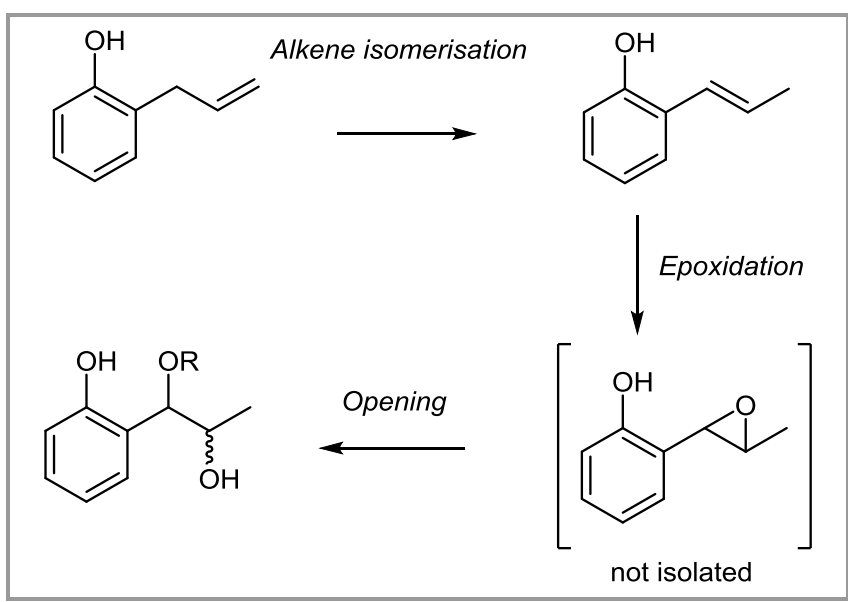

Scheme 67 Proposed pathway for dioxygenation of 2-allylphenols.

The authors noticed that a background reaction was taking place when 2-(propen-1-yl)phenol was used as substrate without the palladium catalyst (Scheme 68). The reaction afforded the 1,2-dialkoxylated products with very low conversion and required a longer reaction time, indicating the contribution of the metal catalyst in the process (a). The phenol moiety appeared to be an essential feature of the substrate, as reactions of allylbenzene and 2-allylanisole led only to a mixture of Wacker oxidation and alkene isomerisation products (b). These observations were supported by the studies of Jacobs et al. on the metalfree and phenol-mediated epoxidation of alkenes in the presence of hydrogen peroxide. ${ }^{106}$

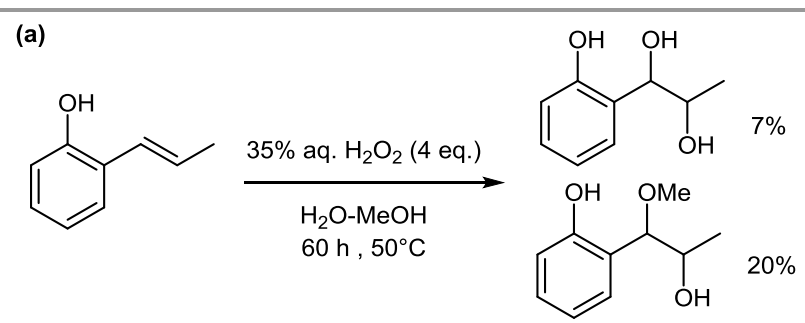

(b)

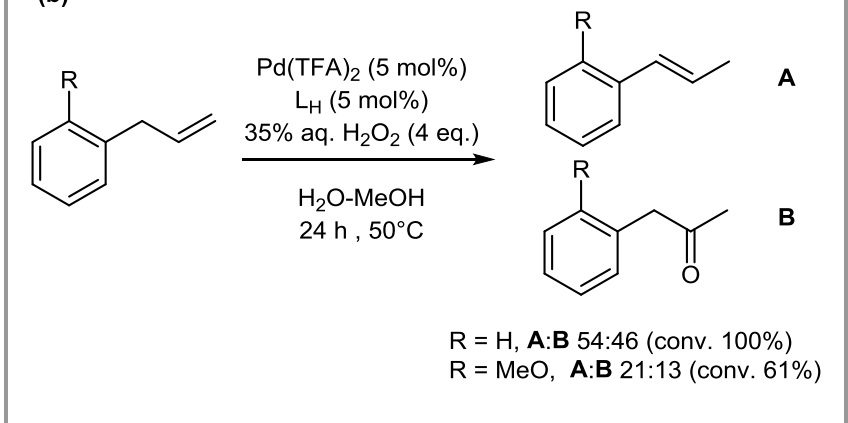

Scheme 68 Experiments to probe the dihydroxylation mechanism.

Soon after, Sigman and co-workers published an efficient catalytic system for the dialkoxylation of styrenes derived from ortho-substituted phenols (Scheme 69). ${ }^{107}$ In this procedure, $\mathrm{Pd}\left(\mathrm{CH}_{3} \mathrm{CN}\right)_{2} \mathrm{Cl}_{2}$ was used as a catalyst without additional ligands, and $\mathrm{CuCl}_{2}$ as the oxidant to regenerate the $\mathrm{Pd}(\mathrm{II})$ active species. The copper salt was employed in catalytic amounts with oxygen (1 atm) as the terminal oxidant. 
The reaction occurred at room temperature and afforded the 1,2-dialkoxylated product as a mixture of two diastereomers with modest selectivity. The system was efficient for electron rich styrenes but less effective for electron-poor styrene derivatives, which gave cyclic ketal products instead. The presence of a phenol moiety was absolutely necessary for the reaction to occur as 2-(propenyl)-anisole only gave Wacker oxidation products under the same conditions. Based on careful labeling experiments, the group ruled out the possibility of $\beta$-hydride elimination occurring during the transformation. Instead, a mechanism was proposed involving the formation of a quinone methide intermediate after regioselective alkoxypalladation with addition of $\mathrm{MeOH}$ to the $\beta$ carbon of the styrene.

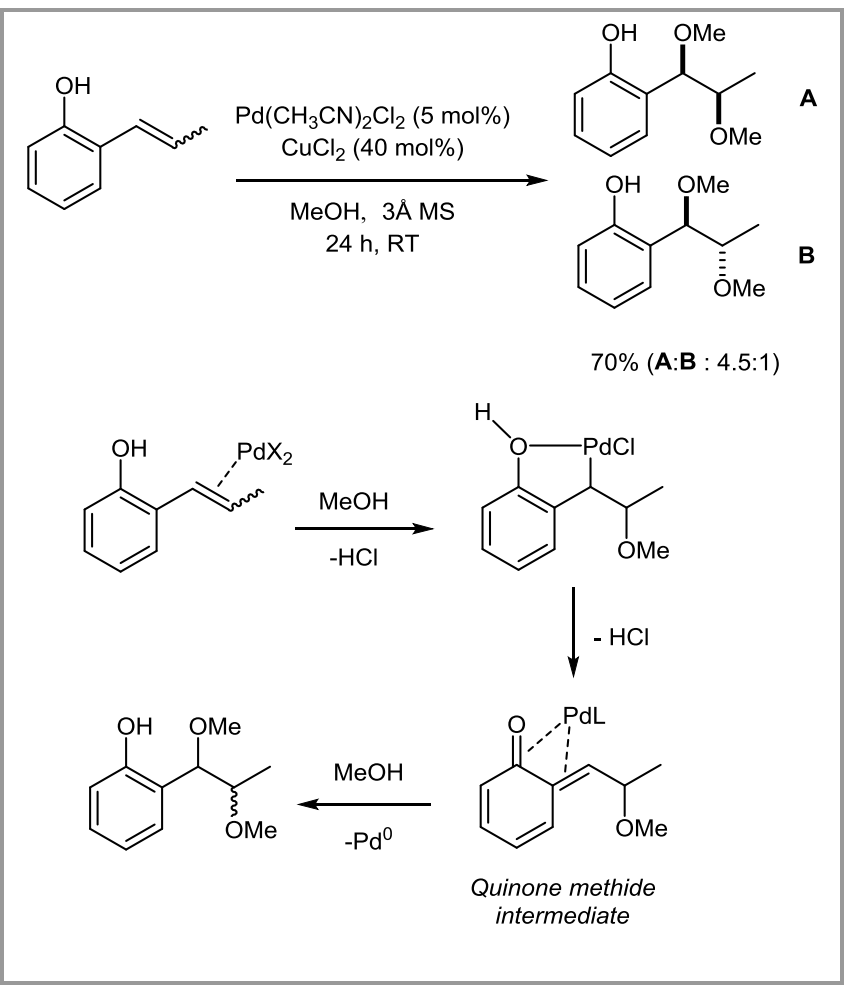

Scheme 69 Dialkoxylation of 2-allylphenols using palladium catalysis and the proposed mechanism.

On the basis of their mechanistic proposal, Sigman et al. saw an opportunity to develop an asymmetric version of this reaction (Scheme 70). ${ }^{108}$ $\mathrm{Pd}\left(\mathrm{CH}_{3} \mathrm{CN}\right)_{2} \mathrm{Cl}_{2}$ remained the metal precursor of choice for this transformation and was combined with a chiral Quinox ligand to induce asymmetry. ${ }^{109} \mathrm{~A}$ remarkable additional benefit of this new procedure was the use of oxygen as the sole oxidant in a copperfree system. In fact the addition of copper salts was observed to lead to lower enantioselectivities. Under the optimised conditions, 1,2-dialkoxylated products were obtained in moderate yields and promising enantioselectivities (i.e. up to $92 \%$ ee).

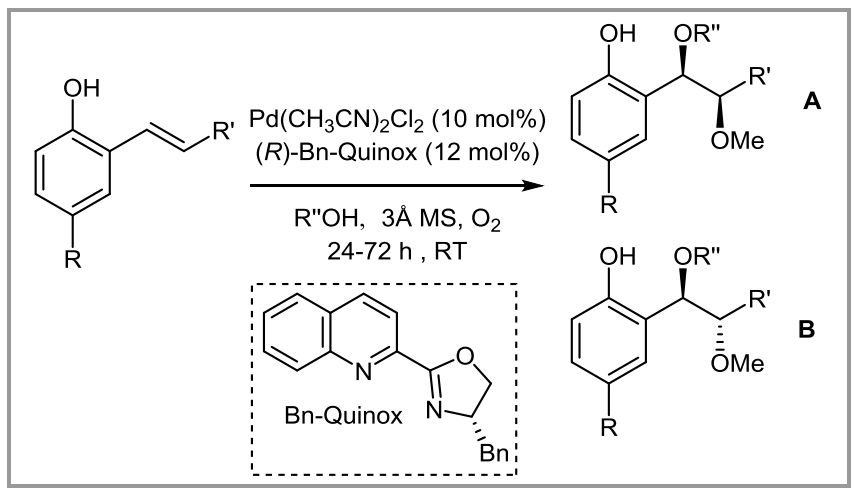

Scheme 70 Asymmetric 1,2-dioxygenation of 2-allylphenols using a chiral $\operatorname{Pd}($ II)/Quinox system.

\begin{tabular}{cccccc}
$\mathbf{R}$ & $\mathbf{R}$ & $\mathbf{R}$ 'OH & Yield & ee & A:B \\
\hline $\mathbf{H}$ & $\mathrm{Me}$ & $\mathrm{MeOH}$ & 63 & 85 & $5: 1$ \\
\hline $\mathbf{O M e}$ & $\mathrm{Me}$ & $\mathrm{MeOH}$ & 74 & 92 & $7.5: 1$ \\
\hline $\mathbf{M e}$ & $\mathrm{Me}$ & $\mathrm{MeOH}$ & 70 & 91 & $8: 1$ \\
\hline $\mathbf{C l}$ & $\mathrm{Me}$ & $\mathrm{MeOH}$ & 60 & 86 & $5: 1$ \\
\hline $\mathbf{H}$ & $\mathrm{Et}$ & $\mathrm{MeOH}$ & 64 & 82 & $6: 1$ \\
\hline $\mathbf{H}$ & $\mathrm{Me}$ & $\mathrm{HO}\left(\mathrm{CH}_{2}\right)_{2} \mathrm{OH}$ & 46 & 81 & $7: 1$ \\
\hline
\end{tabular}

A few years later, after a substantial mechanistic study, the same group rationalised the detrimental effect of copper salts on enantioselectivity. ${ }^{110} \mathrm{~A}$ rapid ligand exchange between the two metal precursors was taking place, allowing the achiral Pd(II) complex formed in situ to act as catalyst which reduced selectivity. They then evaluated potential catalytic systems using palladium and copper precursors in conjunction with an excess of chiral ligand and finally achieved oxidation with a shorter reaction time, higher conversion and similar enantioselectivities to the direct $\mathrm{O}_{2}$-coupled oxidation.

It was not until 2009 that the first methodology based on a $\mathrm{Pd}(\mathrm{II}) / \mathrm{Pd}(\mathrm{IV})$ strategy for alkene dioxygenation was developed, by Dong and co-workers (Scheme 71). ${ }^{111}$ After optimisation, the refined set of conditions employed a cationic palladium complex with a diphosphine ligand (dppp) combined with an iodonium salt as oxidant. The reaction takes place in acetic acid at $50{ }^{\circ} \mathrm{C}$ and afforded 1-hydroxy-2acetoxy- products in good yield with modest to high syn selectivities. Diacetoxylated products were obtained by further treatment of the reaction mixture with acetic anhydride.

The substrate scope of this reaction is quite broad with terminal (a), internal, aromatic and aliphatic alkenes being converted into the dioxygenated compounds in good yields. Electron poor alkenes were also well tolerated. 1,2-Disubstituted olefins were also converted in good yield and high diastereoselectivity (b), and 1,1-disubtituted alkenes were oxidised in good yields to afford exclusively tertiary alcohols (c). It is also interesting to note that this procedure can be applied analogously in an intramolecular fashion to afford an oxygenated heterocycle in good yield. The mechanism for the reaction (Scheme 72) was 
proposed to occur via a trans-acetoxypalladation followed by oxidation of the metal centre to $\mathrm{Pd}(\mathrm{IV})$ with the iodonium salt. Finally, intramolecular cyclisation gives an acetoxonium intermediate, which is hydrolysed to give the syn-oxygenated product and regenerate the $\mathrm{Pd}(\mathrm{II})$ species.

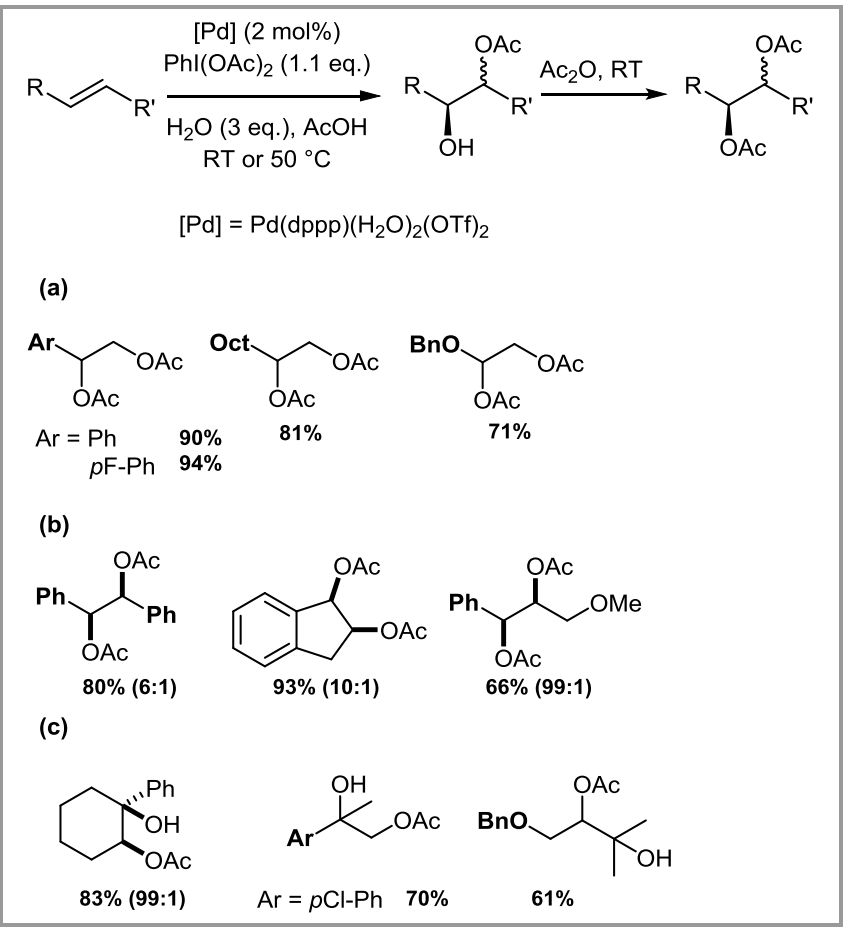

Scheme 71 Palladium-catalysed dioxygenation of alkenes.

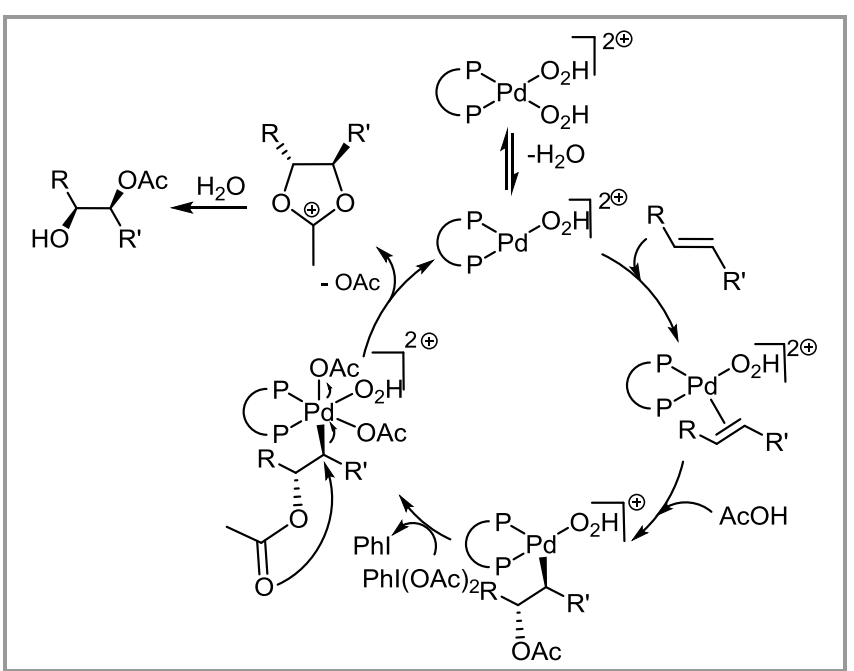

Scheme 72 Proposed mechanism for the $\mathrm{Pd}(\mathrm{II}) / \mathrm{Pd}(\mathrm{IV})$ alkene dioxygenation.

Shortly afterwards, Jiang and co-workers reported a novel palladium catalysed diacetoxylation of alkenes using oxygen as the sole oxidant (Scheme 73). ${ }^{112}$ The catalytic system involved $\mathrm{Pd}(\mathrm{OAc})_{2}$ as the palladium source and $\mathrm{KI}$ as additive. The reaction takes place at $100{ }^{\circ} \mathrm{C}$ for $24 \mathrm{~h}$ in acetic acid under $5-8 \mathrm{~atm}$ of oxygen. Diacetate products are prepared in high yields from aliphatic or aromatic terminal olefins (a) as well as internal alkenes (b). Moreover when 1,2disubtituted alkenes were used as substrates, the reaction afforded the corresponding oxidation products with high selectivity for the syn diastereoisomer.<smiles>[R]C=CC=C[R]([H])([H])[O-]</smiles>

Scheme 73 Direct oxygen-coupled alkene-diacetoxylation via palladium catalysis.

The authors carried out experiments to elucidate the mechanism of this transformation, and suggested a $\mathrm{Pd}(\mathrm{II}) / \mathrm{Pd}(\mathrm{IV})$ catalytic cycle with a first step of synacetoxypalladation followed by oxidation to reach a high valent $\mathrm{Pd}(\mathrm{IV})$ intermediate (Scheme 74). Subsequent reductive elimination would regenerate the $\mathrm{Pd}(\mathrm{II})$ active species with concomitant formation of a new C-O bond with syn selectivity. However, another possible explanation is a pathway involving an anti-nucleopalladation followed by functionalisation of the $\mathrm{C}-\mathrm{Pd}$ bond with inversion (vide infra, Scheme 77). A $\mathrm{Pd}(0) / \mathrm{Pd}(\mathrm{II})$ pathway was ruled out since an alkene treated with a stoichiometric amount of $\mathrm{Pd}(\mathrm{OAc})_{2}$ in acetic acid was not converted to the 1,2-diacetate. Obviously, more evidence is required to confirm this hypothesis as oxidation of $\mathrm{Pd}(\mathrm{II})$ to $\mathrm{Pd}(\mathrm{IV})$ with oxygen has never been previously observed. The role of potassium iodide also remained unclear. In fact when a control experiment was carried out with iodine as oxidant (without oxygen) no conversion was observed. This result showed that the iodine generated in situ is not responsible for the palladium oxidation. Instead the authors suggested that the iodide could act as a ligand which promotes the oxidation of $\mathrm{Pd}(\mathrm{II})$ to $\mathrm{Pd}(\mathrm{IV})$. 


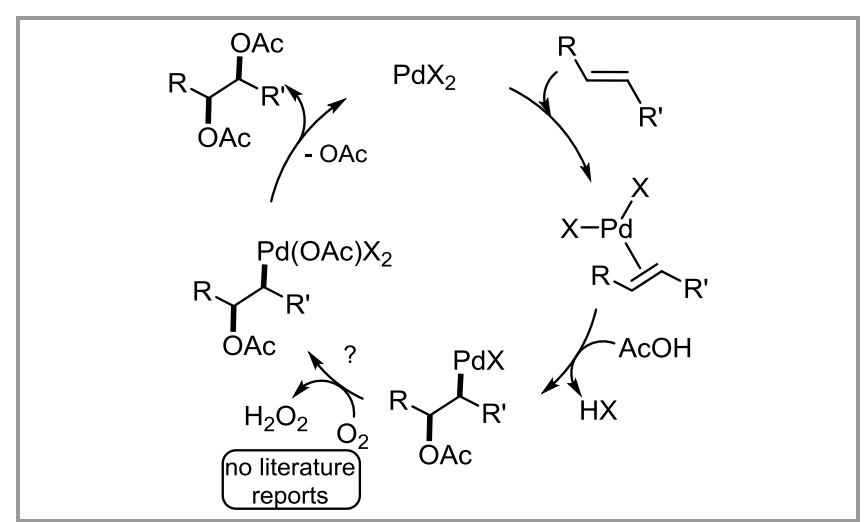

Scheme 74 Proposed mechanism for syn-1,2-diacetoxylation of alkenes using molecular oxygen as the terminal oxidant.

Soon afterwards, the same group reported another contribution to the field with a modified procedure for the preparation of 1,2-diols under aerobic conditions (Scheme 75). ${ }^{113}$ The reaction was performed in water using $\mathrm{Pd}(\mathrm{OAc})_{2}$ as catalyst under basic conditions with oxygen $(8 \mathrm{~atm})$ as oxidant. The presence of base is essential to afford the diol product, as under acidic conditions the alkene is oxidatively cleaved. In contrast with their previous methodology, potassium iodide was not needed as an additive in this reaction.

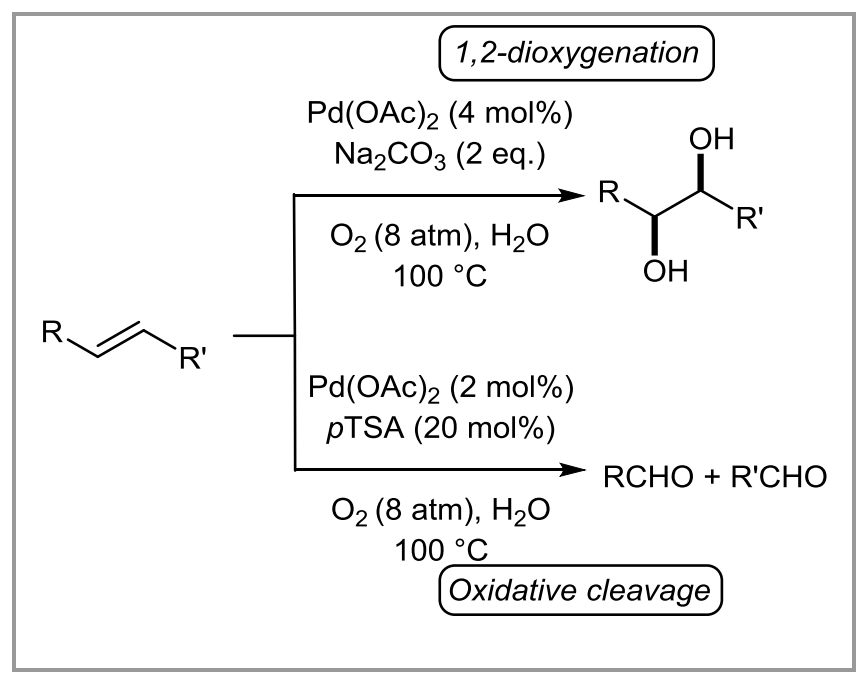

Scheme 75 Pd-catalysed 1,2-dihydroxylation of alkenes under basic conditions. Oxidative cleavage of the alkene was observed under acidic conditions.

In the same year, Shi and co-workers reported a novel procedure for the diacetoxylation of alkenes via palladium catalysis using $N$-heterocyclic carbene (NHC) ligands (Scheme 76). ${ }^{114}$ Taking advantage of the fact that NHCs are known to form robust, air and moisture stable catalysts and also to stabilise high oxidation states, they used an iodonium salt as the oxidant to promote a $\mathrm{Pd}(\mathrm{II}) / \mathrm{Pd}(\mathrm{IV})$ catalytic cycle. ${ }^{115}$ The reaction occurs at $100{ }^{\circ} \mathrm{C}$ in anhydrous acetic acid with a cationic palladium-biscarbene complex. When wet acetic acid was used, better conversions were achieved, although the diacetate product was obtained along with a high proportion of syn-hydroxyacetate. Most of the substrates were converted in high yields with moderate diastereoselectivities.

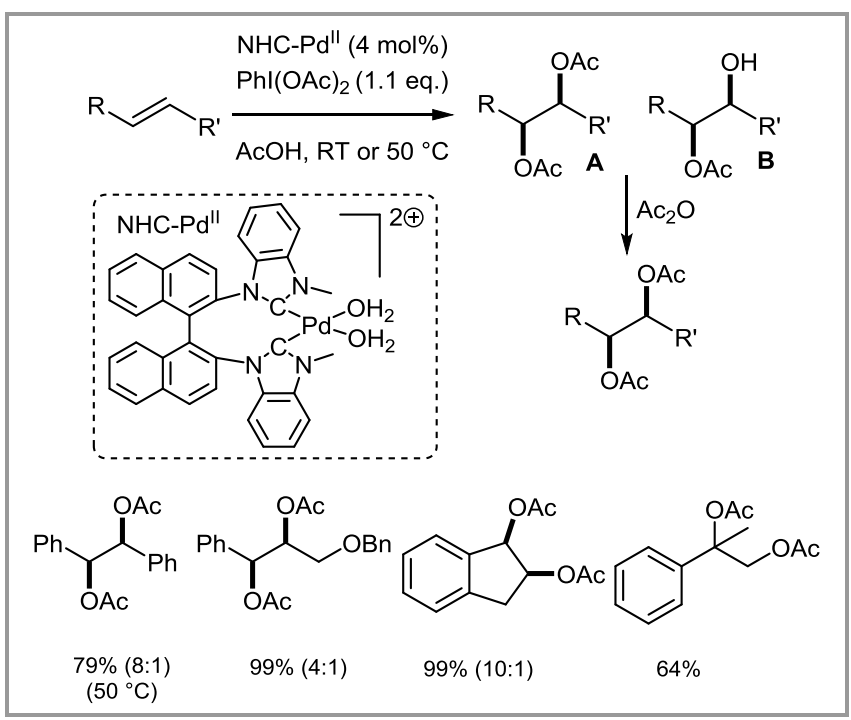

Scheme 76 Palladium-catalysed diacetoxylation of alkenes using $\mathrm{N}$-heterocyclic carbene ligands.

Formation of the hydroxyacetate by-product was rationalised by the same mechanism proposed by Dong and co-workers, ${ }^{111}$ via a three step sequence induced by a trans-acetoxypalladation (Scheme 77). Following this, the palladium complex formed is oxidised to $\mathrm{Pd}(\mathrm{IV})$ with the iodonium salt and finally intramolecular cyclisation affords the acetoxonium intermediate and regenerates the $\mathrm{Pd}(\mathrm{II})$ active species. Further hydrolysis released the syn-hydroxyacetate product. The high syn-selectivity observed under Shi's conditions is in total agreement with this mechanism. The authors suggested that both hydroxyacetate and diacetate compounds likely arise from the same palladium(IV) species. Formation of the hydroxyacetate product was explained by neighbouring group participation of the adjacent acetate which can directly displace the $\mathrm{Pd}(\mathrm{IV})$ to give an oxonium ion that is subsequently hydrolysed to the syn-hydroxyacetate (a). To rationalise the synselectivity observed for the 1,2-diacetate product (b), Shi et al. suggested an " $\mathrm{S}_{\mathrm{N}} 2$-type" elimination with inversion of configuration resulting from an intramolecular or intermolecular attack of an acetate ligand (Pathway I). In contrast, a concerted reductive elimination process at the metal centre would be expected to lead to the anti-product (Pathway II). 


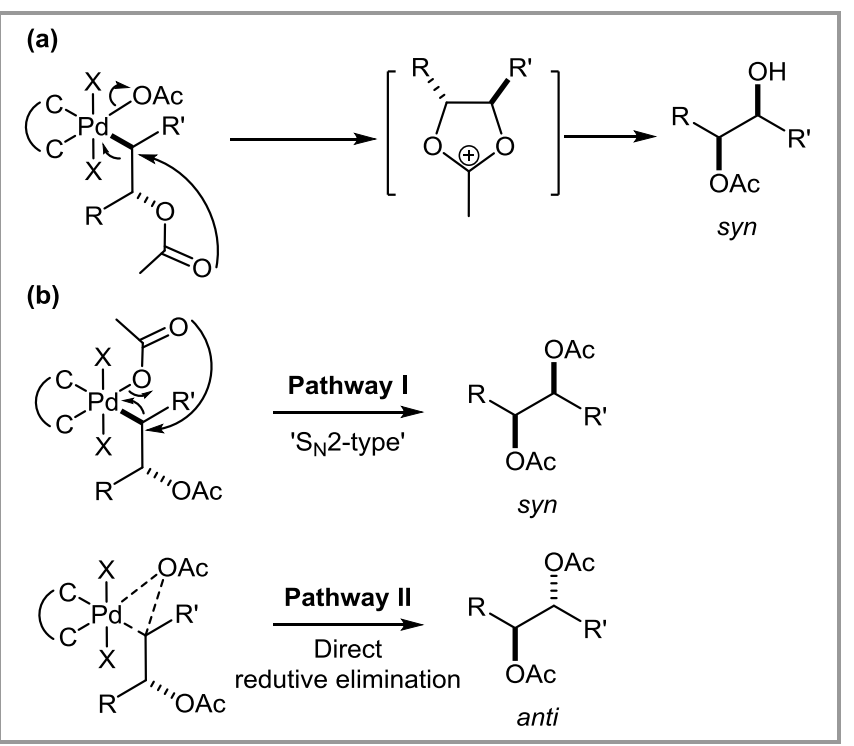

Scheme 77 Proposed pathways for Pd-catalysed diacetoxylation via a $\mathrm{Pd}(\mathrm{II}) / \mathrm{Pd}(\mathrm{IV})$ cycle.

More recently, a contribution by Jung, Park et al. reported the diacetoxylation of alkenes using $\mathrm{Pd}(\mathrm{OAc})_{2}$ as catalyst and peracetic acid as oxidant in the presence of acetic anhydride (Scheme 78). ${ }^{116}$ The difunctionalisation occurred under mild conditions at room temperature in anhydrous acetic acid. The reaction was rapid and can efficiently be applied to a small range of aliphatic and aromatic internal and terminal alkenes with high diastereoselectivity in favour of the syn-product.

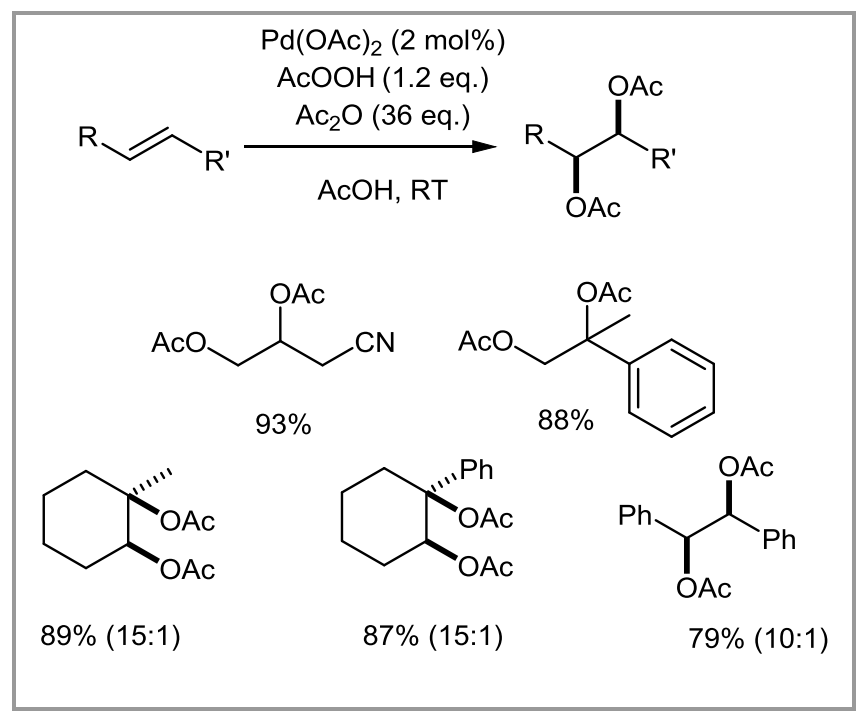

Scheme 78 Palladium-catalysed diacetoxylation using peracetic acid as the oxidant.

The mechanism proposed for this reaction involved a $\mathrm{Pd}^{\mathrm{IV}}(\mathrm{OAc})_{4}$ intermediate generated by oxidation of $\mathrm{Pd}(\mathrm{OAc})_{2}$ with peracetic acid in the presence of acetic anhydride. Activation of the olefin by coordination to the $\mathrm{Pd}(\mathrm{IV})$ centre enables syn-nucleopalladation followed by reductive elimination, leading to the product along with $\mathrm{Pd}(\mathrm{II})$. A slightly modified version of this method was developed by Park and co-workers using a micro-reactor. ${ }^{117}$ High yields were obtained in very short reaction times at $60{ }^{\circ} \mathrm{C}$, but the process was only tested on three different alkenes and was not investigated further.

In 2013, Sanford et al. reported a palladium catalysed asymmetric methodology for alkene dibenzoylation (Scheme 79). ${ }^{118}$ Their strategy relies on the introduction of a chiral auxiliary into the substrate to induce higher reactivity and selectivity. Chiral oxime ethers were used as directing groups as they are readily available and known as potential directing ligands in palladium catalysis. The reaction conditions involved $\mathrm{Pd}(\mathrm{PhCN})_{2} \mathrm{Cl}_{2}$ as palladium source combined with an iodonium salt. Reactions were performed in dry toluene at $50{ }^{\circ} \mathrm{C}$. The results were dependent on the chiral allyl oxime ether used, with the reactivity being highly dependent on the steric bulk of the directing group; when sterically hindered auxiliaries were employed, conversion declined but the diastereoselectivity increased. The authors reported that the best balance between yield and selectivity was obtained with auxiliaries derived from menthone.

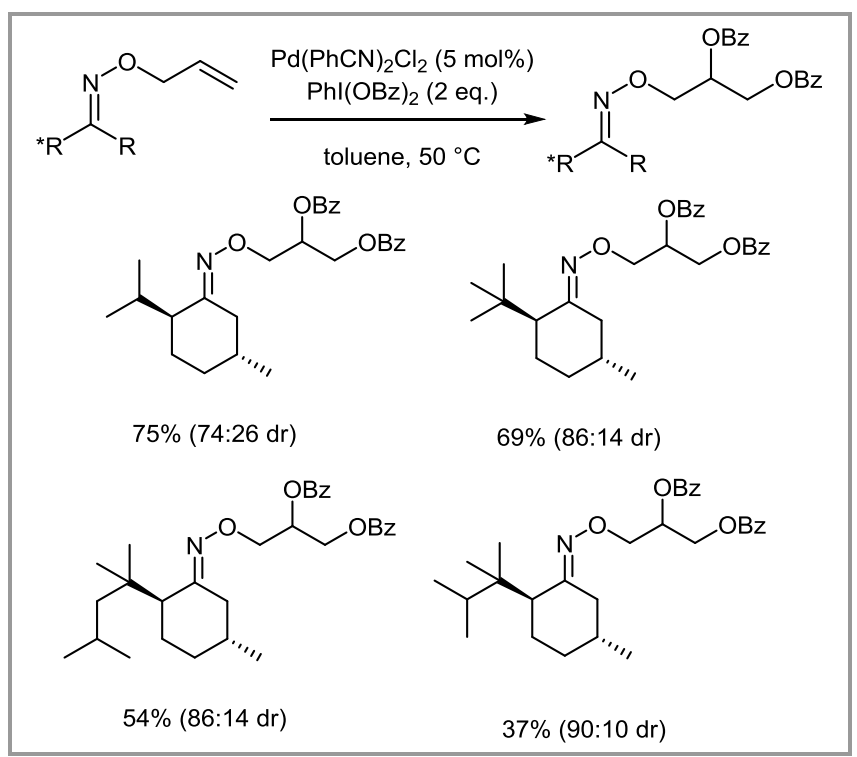

Scheme 79 1,2-Dibenzoylation of oxime allyl ethers.

Based on literature precedent, the authors suggested a mechanism initiated by coordination of the oxime ether prior to oxypalladation as simple allyl ethers (e.g. allyl propyl ether) were unreactive under the reaction conditions. The Pd-alkyl species is then oxidised to $\mathrm{Pd}(\mathrm{IV})$ to promote the second $\mathrm{C}-\mathrm{O}$ bond formation. When internal olefins were employed, dibenzoylation proceeded with high selectivity for the syn-product suggesting two possible pathways for the transformation (Scheme 80). The reaction could be initiated by an anti-oxypalladation step followed by oxidation and $\mathrm{C}-\mathrm{O}$ formation with inversion of configuration to account for the selectivitites observed. Alternatively, if the oxypalladation is a syn process, the last step would be a direct reductive elimination with retention of configuration. 


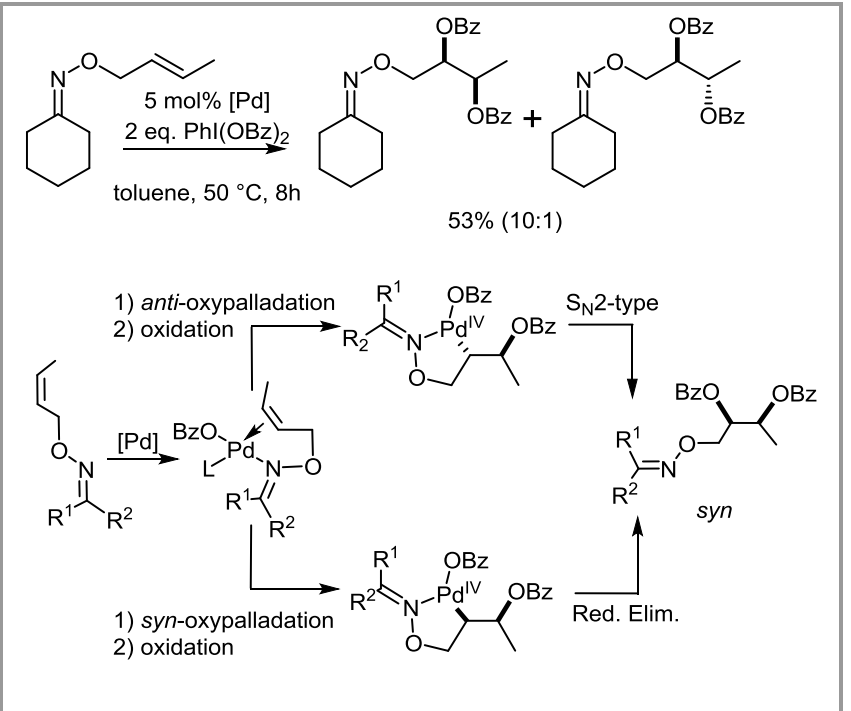

Scheme 80 Possible pathways for the syn-dibenzoylation of oxime ethers.

Finally, very recently Grubbs et al. have reported a novel methodology for the palladium catalysed intermolecular diacetoxylation of terminal alkenes under aerobic conditions (Scheme 81). ${ }^{119}$ In a similar way to a related Wacker oxidation procedure (vide infra, Scheme 50), the transformation relies on the use of a catalytic amount of silver nitrite in combination with a $\mathrm{Pd}(\mathrm{PhCN})_{2} \mathrm{Cl}_{2}$ catalyst and a $\mathrm{Cu}(\mathrm{II})$ co-catalyst. The reaction was performed in a complex mixture of acetic acid, acetic anhydride and nitromethane (10:5:3) at $35{ }^{\circ} \mathrm{C}$ under an oxygen atmosphere (1 atm). Under the reaction conditions aliphatic alkenes were converted in moderate to excellent yields into the corresponding diacetates, and a variety of functional groups were tolerated (e.g. halide, ester, ether, carboxylic acid, nitro and sulfonamide). The method was also applied on a two-gram scale with comparable efficiency.

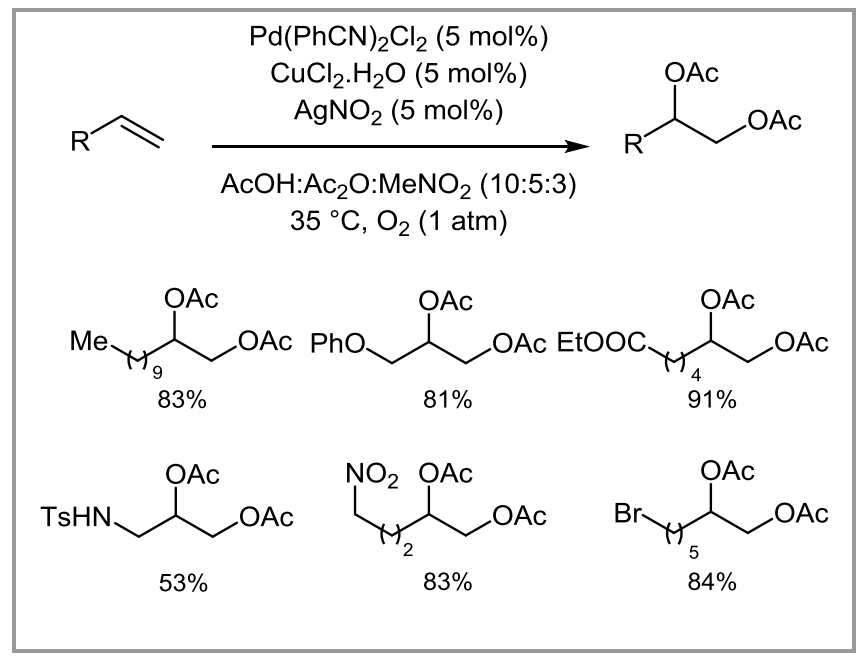

Scheme 81 Pd-catalysed 1,2-diacetoxylation of terminal alkenes using copper chloride and silver nitrite co-catalysts.

Based on their results and some NMR labeling experiments, the authors suggested that the reaction occurred via an initial nucleopalladation step (Scheme
82) to give a $\sigma$-alkyl palladium(II) intermediate which is oxidised to $\mathrm{Pd}(\mathrm{IV})$ by an NOx species generated from the silver nitrite under the reaction conditions. The high-valent palladium complex can undergo an intramolecular reductive elimination to generate an acetoxonium ion in analogy to the mechanism reported previously by Dong and co-workers. ${ }^{111}$ Finally, subsequent hydrolysis affords the diacetoxylated product. It is worth noting that this mechanism did not clarify the role of the copper cocatalyst which appeared to be essential for efficient turnover.

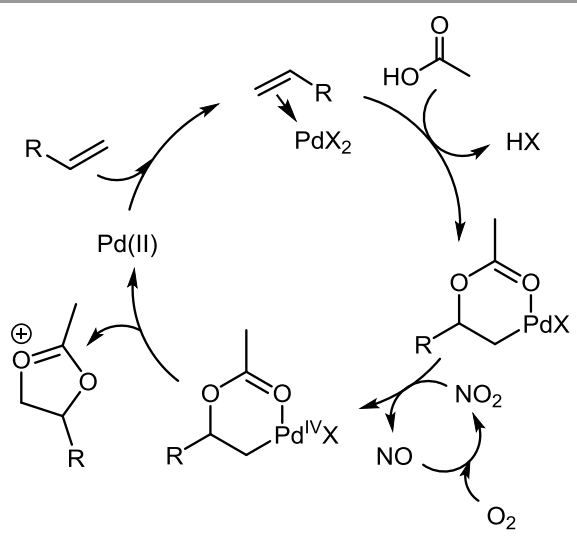

Scheme 82 Possible mechanism for the Pd-catalysed diacetoxylation of alkenes in the presence of silver nitrite.

\subsection{Oxycarbonylation Reactions}

In 1976, Stille et al. reported a method for the direct methoxycarbonylation of alkenes in methanol using a $\mathrm{PdCl}_{2} / \mathrm{CuCl}_{2}$ catalytic system under a carbon monoxide atmosphere (Scheme 83). ${ }^{120}$ Under the same conditions, cyclic olefins gave predominately the corresponding 1,2-diesters or 1,3-diesters. This latter product was proposed to be generated via isomerisation of the initially formed $\sigma$-organopalladium intermediate via $\beta$-hydride elimination and then hydropalladation. Intramolecular alkoxycarbonylation reactions have subsequently been developed by other researchers for the synthesis of a wide range of oxygen heterocycles. ${ }^{121}$ 


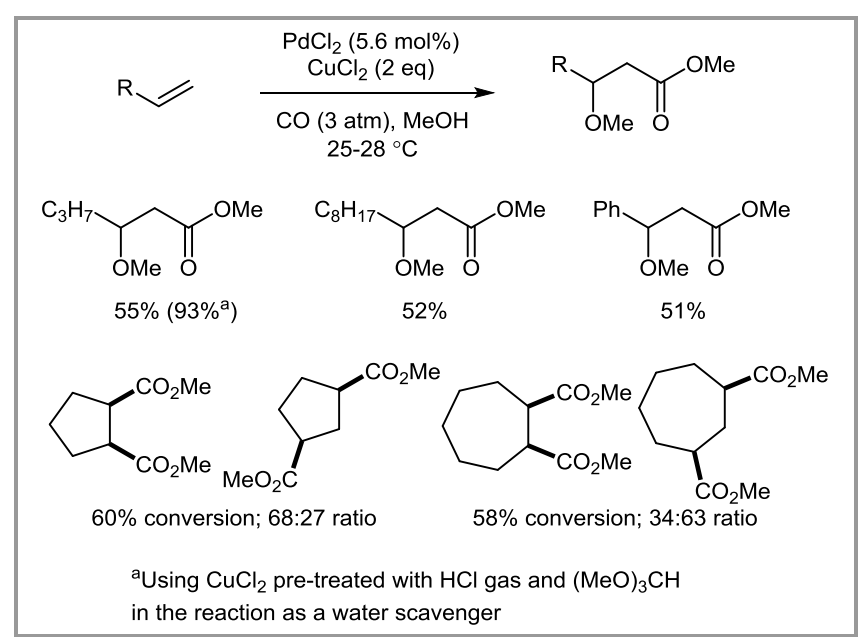

Scheme 83 Pd-catalysed methoxycarbonylation of terminal alkenes and dicarbonylation of cyclic alkenes.

\subsection{Aminohalogenation Reactions}

In 2009, Liu et al. reported a methodology to access fluorinated cyclic amines via an intramolecular palladium catalysed aminofluorination of olefins. ${ }^{122}$ Taking their research one step further, in 2010 the same group reported an intermolecular variant. ${ }^{123}$ The optimal conditions for the reaction employed $\mathrm{Pd}(\mathrm{OAc})_{2}$ as a catalyst in presence of a bathocuproine ligand. $N$-Fluorobenzenesulfonimide (NFSI) was used as the oxidant/electrophilic fluorine reagent as well as the nitrogen source. The reaction took place in dioxane and the concentration $(0.4 \mathrm{M})$ was found to be an important parameter for high conversions (Scheme 84). The amino-fluorinated compound was obtained as the major product with a small amount of difluorinated amine byproduct. Electron rich aromatic rings as well as haloarenes were tolerated during the process. In contrast, under these conditions strong electron withdrawing substituents on the benzene ring had a detrimental effect on the conversion. Internal alkenes derived from styrene were also converted in good yields but only low diastereoselectivities were observed. The authors proposed a complex mechanism involving $\operatorname{Pd}(0), \operatorname{Pd}($ II) and $\mathrm{Pd}(\mathrm{IV})$ species. An unusual fluoropalladation of the alkene was suggested to account for the formation of both the major product and the difluorinated byproduct. However, such a pathway would not be expected to proceed with the observed regioselectivity based on other well known processes involving nucleopalladation of styrenes such as the Wacker oxidation. An initial aminopalladation, followed by fluorination of the resulting Pd-alkyl bond would be a feasible explanation for the formation of the major product. It is possible, however, that the difluorinated product arises from the formation of an enamine intermediate through $\beta$-hydride elimination from the alkylpalladium intermediate.

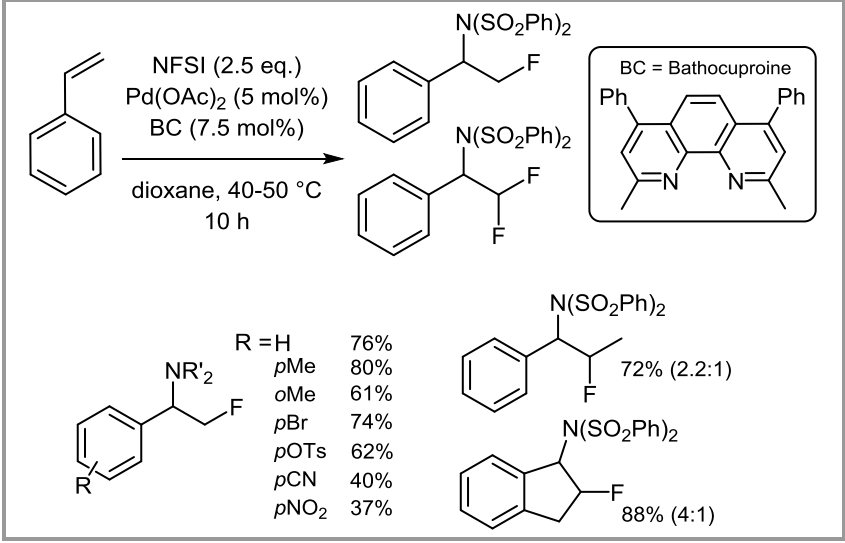

Scheme 84 Pd-catalysed aminofluorination of styrenes.

\subsection{Diamination Reactions}

Purely intermolecular diamination of alkenes remains a challenge in palladium catalysis and only a limited numbers of reports have appeared in this area.

The first palladium-promoted formal diamination of an olefin was reported in 1977 by Bäckvall and coworkers, who presented a synthesis of aziridines via a palladium-mediated strategy (Scheme 85). ${ }^{124}$ The reaction was carried out at low temperature with a stoichiometric amount of palladium, methylamine as nucleophile and bromine as oxidant. Under these conditions the aziridines were obtained in modest yields. The reaction was proposed to be initiated by trans-aminopalladation, followed by oxidation of the resulting $\beta$-amino-alkylpalladium complex with bromine to form a $\mathrm{Pd}(\mathrm{IV})$ complex. Subsequent displacement of the metal centre by the nitrogen leads to a second $\mathrm{C}-\mathrm{N}$ bond formation and cyclisation to release the heterocycle. Shortly afterwards, a slightly modified procedure was reported by the same group for the vicinal diamination of alkenes. ${ }^{125}$ In this specific case, the nucleopalladated intermediate was oxidised (with $\mathrm{Br}_{2}, m \mathrm{CPBA}$ or $\mathrm{Pb}(\mathrm{OAc})_{4}$ ) in the presence of a secondary amine to afford the diamine compound.

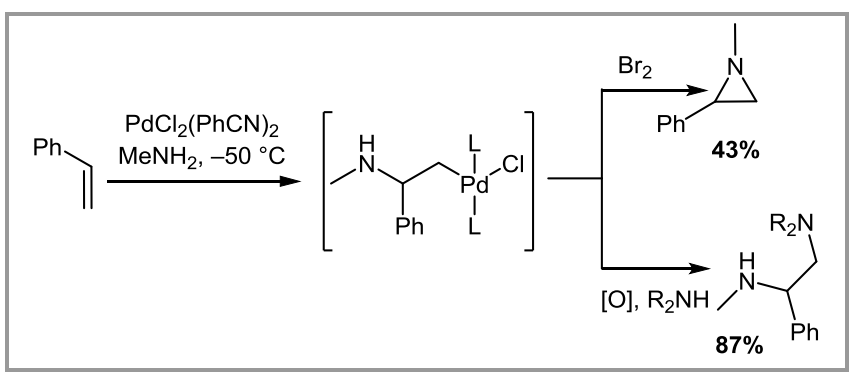

Scheme 85 Pd-mediated aziridination and 1,2-diamination of alkenes.

After this pioneering report, it was not until 2001 that Branco and co-workers published a catalytic synthesis of aziridines from olefins with bromamine- $T$ as the nitrogen source (Scheme 86). ${ }^{126}$ The procedure still involved a high palladium loading with 0.2 eq. of $\mathrm{PdCl}_{2}$. However, the transformation was very effective for the aziridination of $\mathrm{N}, \mathrm{N}$-dimethylacrylamide at room temperature. The transformation was proposed 
to take place via a $\mathrm{Pd}(\mathrm{IV})$ complex, formed after an intramolecular oxidative addition of the $\sigma$-alkylpalladium intermediate. Finally, reductive elimination affords the aziridine.

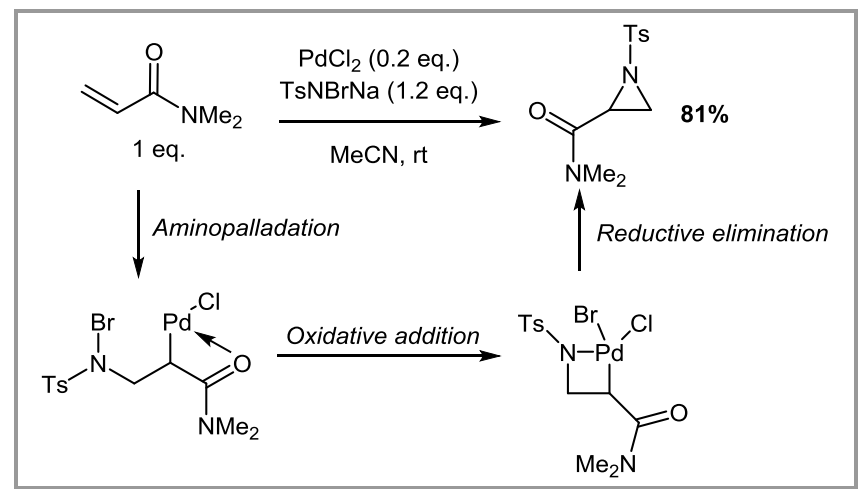

Scheme 86 Pd-catalysed aziridination of alkenes using bromamine-T as the oxidizing agent.

Several years later, Pan, Li et al. presented a methodology for the aziridination of olefins involving a fairly low catalyst loading $(2 \mathrm{~mol} \%) .{ }^{127}$ The nitrogen transfer agent used was $N, N$-dichloro- $p$ toluenesulfonamide, readily prepared from tosylamine. The presence of a base $\left(\mathrm{K}_{2} \mathrm{CO}_{3}\right)$ was required to cyclise the initially formed aminohalogenation product and afford the aziridine heterocycle in moderate yields (Scheme 87).

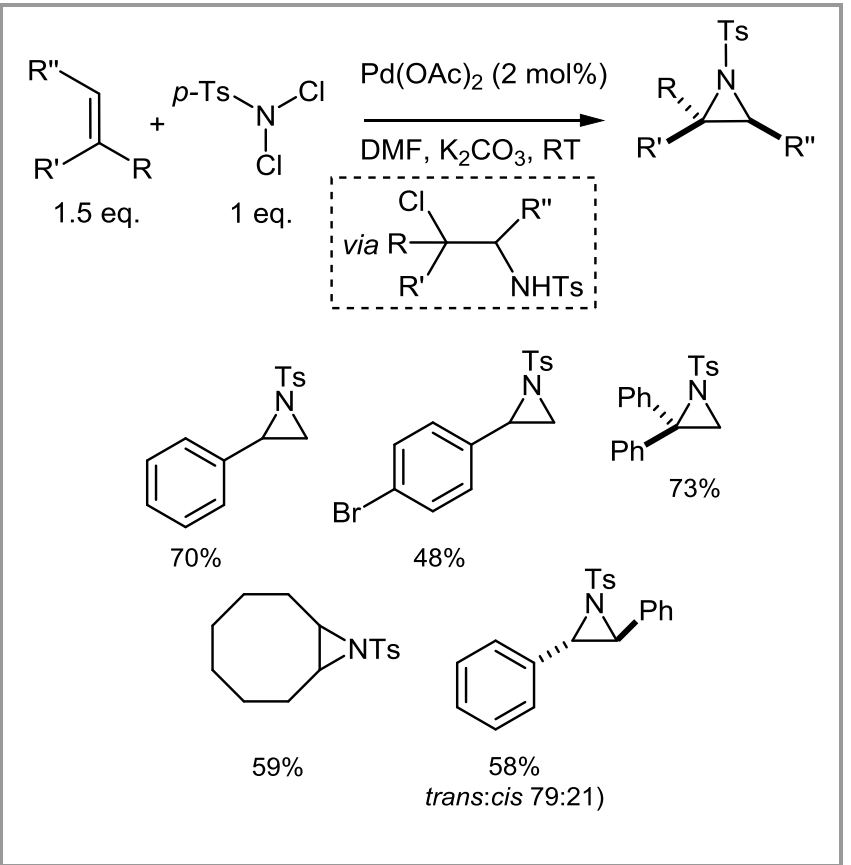

Scheme 87 Pd-catalysed aziridination of alkenes via an intermediate aminohalogenation product.

In contrast with previous research on aziridines, Booker-Milburn and Lloyd-Jones reported in 2005 a diamination methodology where two nitrogen atoms were added across the alkene (Scheme 88). ${ }^{128}$ This strategy relied on the use of dienes as activated substrates. $N, N$ '-Dialkylureas were employed as nucleophiles, as they are less prone to coordinating to the palladium centre and deactivating the catalyst. A simple palladium catalyst was employed and benzoquinone was used as the terminal oxidant to reoxidise the $\operatorname{Pd}(0)$ generated.

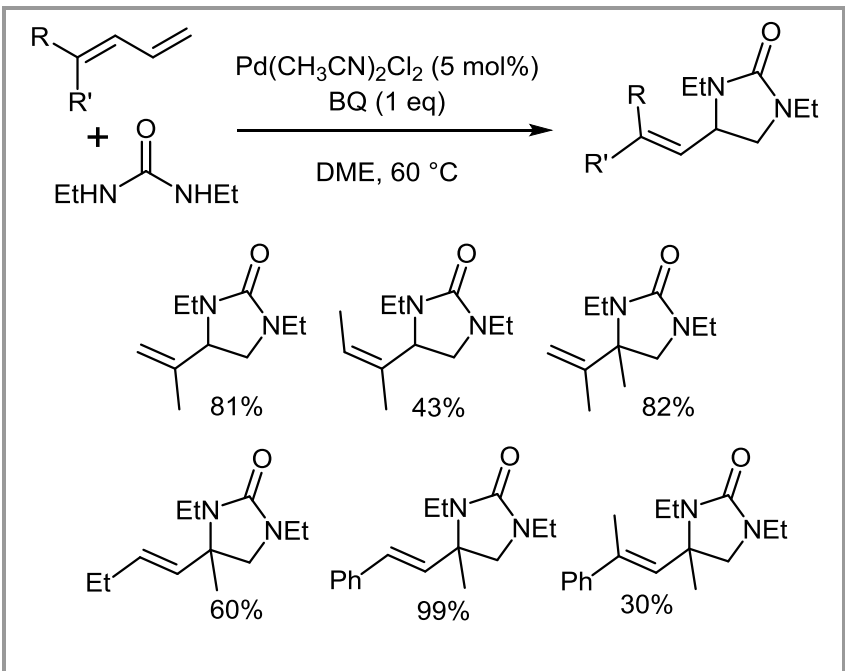

Scheme 88 Pd-catalysed 1,2-diamination of dienes via an intermediate $\pi$-allyl complex.

The transformation was suggested to occur via a $\pi$-allyl Pd intermediate formed after initial aminopalladation (Scheme 89). This electrophilic $\pi$ allyl complex has less tendency to undergo $\beta$-hydride elimination and intramolecular reductive displacement of palladium by the nucleophile takes place instead.

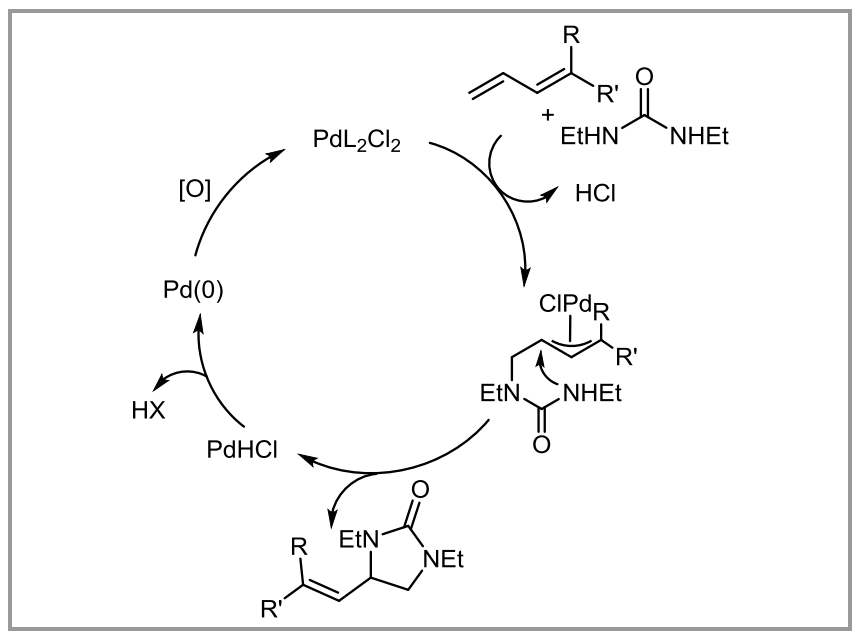

Scheme 89 Proposed mechanism for 1,2-diamination of dienes.

In a similar vein, in 2007 Shi et al. reported a procedure for the diamination of dienes catalysed by a $\operatorname{Pd}(0)$ catalyst with di-tert-butyldiaziridone as the nitrogen source. ${ }^{129}$ Shortly afterwards, the same group reported an asymmetric variant of this reaction using a chiral phosphoramidite ligand to afford diaminated products with high enantioselectivities (Scheme 90). ${ }^{130}$ 


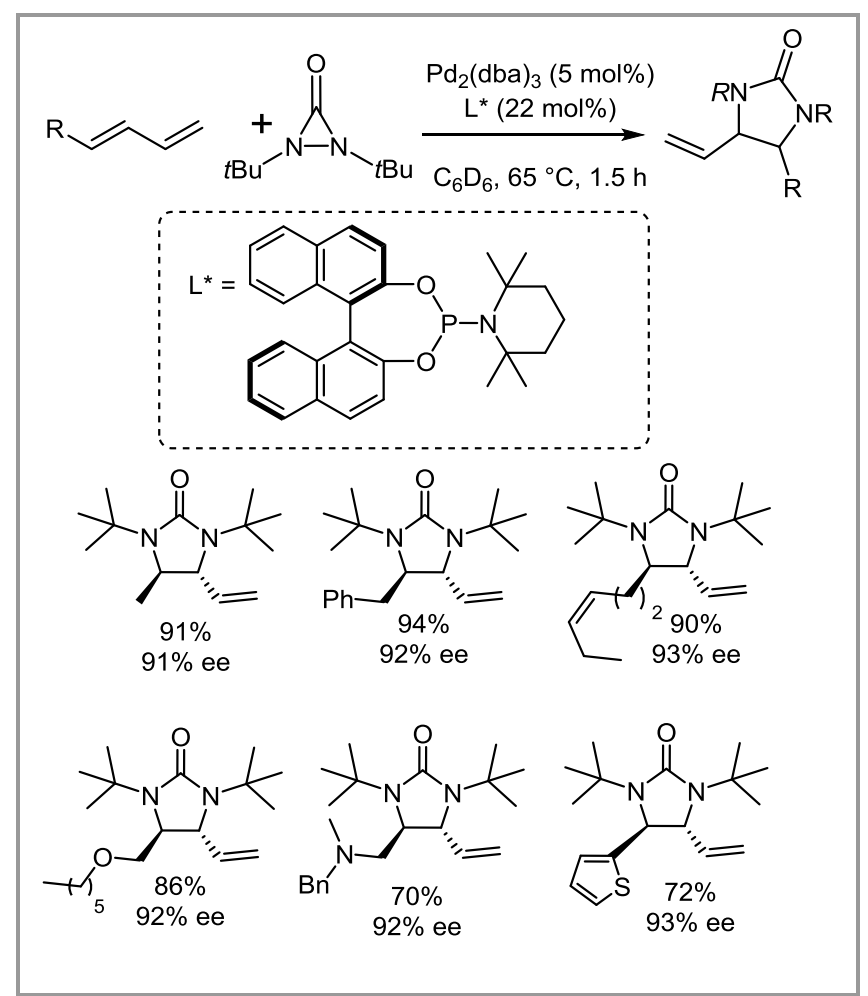

Scheme 90 Pd-catalysed asymmetric 1,2-diamination of dienes using a diaziridine nitrogen source.

The reaction was suggested to involve the formation of a Pd- $\pi$-allyl intermediate via aminopalladation of the diene (Scheme 91). Oxidative addition of the ditert-butyldiaziridone onto the $\operatorname{Pd}(0)$ should afford a 4membered palladacycle which could undergo aminopalladation to generate the Pd-allyl complex. This then undergoes intramolecular displacement to form the product and regenerate $\operatorname{Pd}(0)$. Closely related procedures were also developed for the direct diamination of terminal alkenes by allylic activation and in situ generation of the diene via $\beta$-hydride elimination. ${ }^{131}$

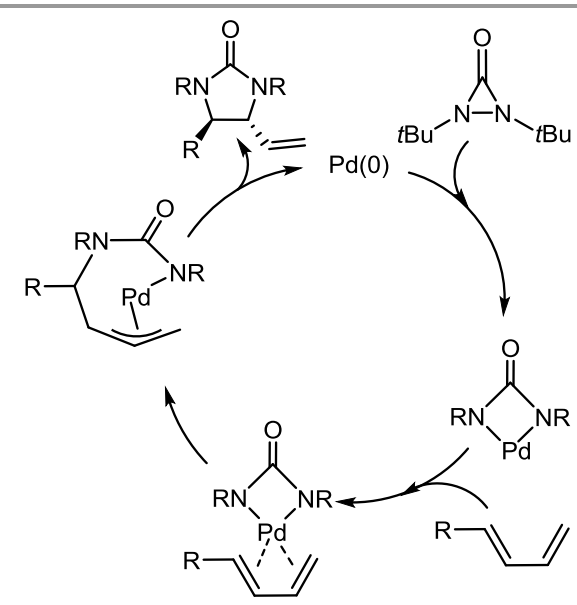

Scheme 91 Proposed mechanism for the asymmetric diamination of dienes.

Another major contribution to this research area was made by Muñiz and co-workers. In line with their studies on the intramolecular diamination of alkenes to access bicyclic ureas, ${ }^{132}$ they reported in 2010 an intermolecular variant of the reaction. ${ }^{133}$ The protocol involved two different commercially available nitrogen sources, saccharin and bis-tosylimide $\left(\mathrm{HNTs}_{2}\right)$. The reaction was sensitive to the palladium source and only $\mathrm{Pd}(\mathrm{RCN})_{2} \mathrm{Cl}_{2}(\mathrm{R}=\mathrm{Me}$, or $\mathrm{Ph})$ were employed with success. The reaction occurred under mild conditions with $5 \mathrm{~mol} \%$ of catalyst at room temperature in the presence of an iodonium salt as oxidant (Scheme 92). Aliphatic terminal alkenes were converted with total chemo- and regioselectivities into the corresponding protected diamines (a). Enamides arising from a $\beta$-hydride elimination process were also observed to a small extent. The reaction conditions proved to be compatible with several functional groups such as esters, amides, azides, malonates, halogens and sulfones. However, internal alkenes were not converted into diamine derivatives under these reaction conditions. Mechanistic investigations were not reported but the reaction is believed to involve a Pd(IV) intermediate. It is also noticeable that the most nucleophilic nitrogen derivative was selectively incorporated during the first nucleopalladation step and the bisulfonimide was involved in the second $\mathrm{C}-\mathrm{N}$ bond formation.

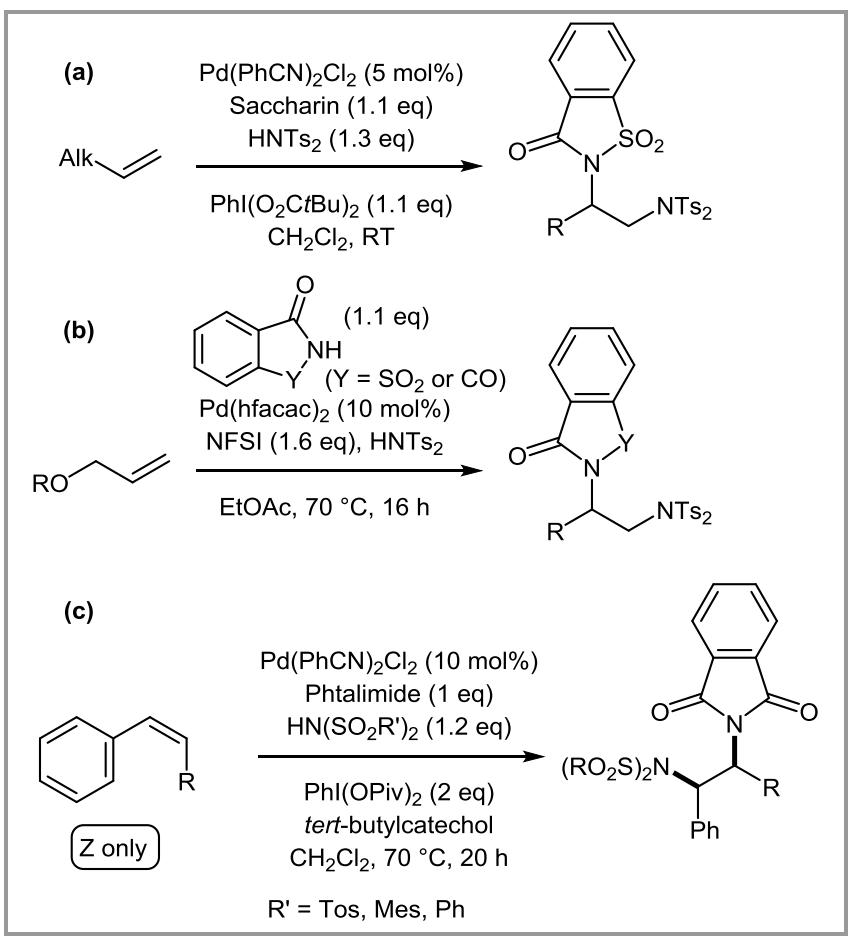

Scheme 92 Recent Pd-catalysed methods for 1,2-diamination of alkenes.

After this initial study, Muñiz et al. extended the methodology to the difunctionalisation of allylic ethers (b). ${ }^{34}$ Electrophilic Pd(hfacac) $)_{2}$ was employed as the palladium precursor combined with phthalimide (or saccharin) and $\mathrm{HNTs}_{2}$ as nitrogen sources in the presence of an iodonium salt. Interestingly, attempts to use NFSI as oxidant and nitrogen source were unsuccessful (low yields) and an external oxidant was required to improve the process. ${ }^{135}$ A series of $O$ - 
alkyl-substituted allyl ethers and allylic alcohols were effectively converted into diamines in good yields. Complete diastereoselectivity was observed for substrates substituted at the allylic position. However, in analogy to their previous methodology, internal alkenes were not reactive under these conditions.

Finally, in 2012, Muñiz et al. achieved the diamination of internal alkenes using a $\mathrm{Pd}(\mathrm{II}) / \mathrm{Pd}(\mathrm{IV})$ strategy (c) ${ }^{136}$ Phthalimide was employed as the first nitrogen donor for the nucleopalladation step. In addition, bis-sulfonimides (tosyl, phenyl or mesyl) were used for the second $\mathrm{C}-\mathrm{N}$ bond formation in the presence of an iodonium salt as oxidant. tertButylcatechol was also employed to avoid polymerisation of the styrene derivatives. Following optimisation, the procedure was applicable on a large scale $(17 \mathrm{mmol})$. Moreover, further transformation of the phthalimide moiety offered easy access to valuable monosulfonylated diamines. (Z)- $\beta$-Alkylstyrenes were transformed into the corresponding diamination products in moderate to high yields but with total regio- and diastereoselectivity. Importantly, only Zalkenes were converted under these reaction conditions and $E$-alkenes were unreactive. Based on these results and on previous mechanistic studies reported for $\mathrm{Pd}(\mathrm{II}) / \mathrm{Pd}(\mathrm{IV})$ catalytic cycles, the authors suggested that the process may occur via precoordination of the olefin to the palladium (Scheme 94). This pre-organisation around the metal centre could explain an anti-aminopalladation process by attack of the phthalimide to form a $\sigma$-alkyl-palladium complex with the metal bonded to the benzylic carbon atom. An oxidation of the $\mathrm{Pd}(\mathrm{II})$ complex in the presence of the iodonium salt would then afford a Pd(IV) intermediate. Finally, metal displacement by the sulfonimide would generate a new $\mathrm{C}-\mathrm{N}$ bond with inversion of configuration.

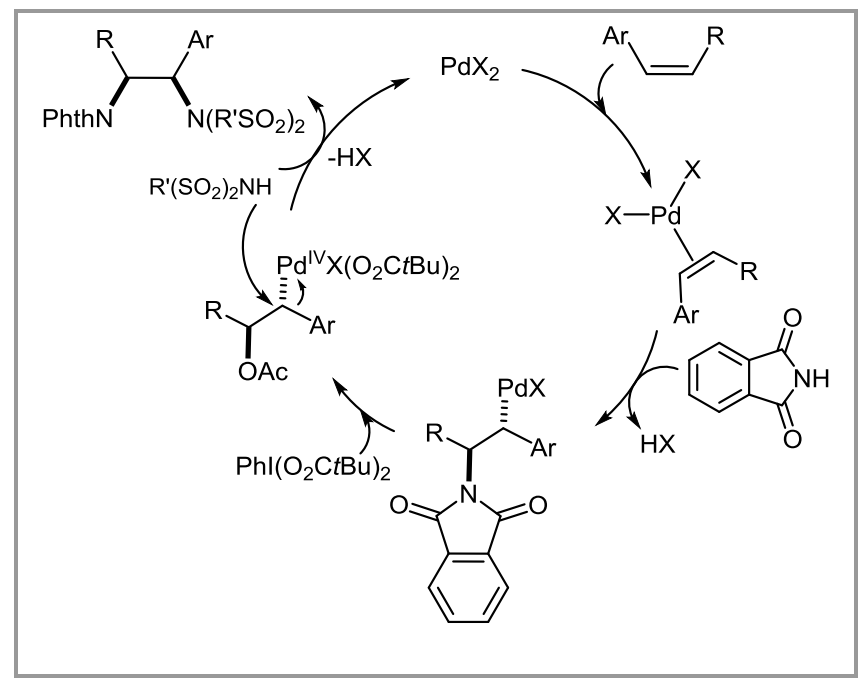

Scheme 94 Proposed mechanism for alkene diamination reactions.

\subsection{Aminooxygenation Reactions \\ Early reports of palladium-mediated} aminoacetoxylation of alkenes were initially published by Bäckvall and co-workers in $1975 .{ }^{137}$ The transformation involved a stoichiometric amount of palladium and led to the aminooxygenated compounds in moderate to good yields. Following this breakthrough, the same group reported in 1982 an asymmetric palladium-promoted oxyamination of alkenes to prepare chiral amino alcohols. ${ }^{138}$ Even though these original reactions were stoichiometric in palladium, this work paved the way for the recent developments in this area. Moreover, many of the mechanistic considerations postulated at the time were verified by later studies.

The first method using catalytic palladium for the aminoacetoxylation of alkenes was reported by Sorensen et al. in 2005, which was an intramolecular process. ${ }^{139}$ A year later Stahl and co-workers described the first intermolecular example (Scheme 95). ${ }^{140}$ The reaction employed phthalimide as the nitrogen source, with a palladium(II) catalyst of choice. An iodonium salt was employed as both the oxidant and as the oxygen nucleophile. Optimisation of the conditions with octene as the substrate led to concomitant formation of vicinal aminoacetylated and diacetoxylated products as well as a $\beta$-hydride elimination product (Wacker-type). The system appeared much more effective for allylic ethers which undergo regioselective aminooxygenation in moderate to good yields. Moreover, substrates with subtitutents on the allylic position were functionalised with high diastereoselectivitity in favour of the anti-isomer in order to avoid steric clash between the allylic substituent and the phthalimide. Unfortunately, internal alkenes were not effectively converted under the typical reaction conditions.

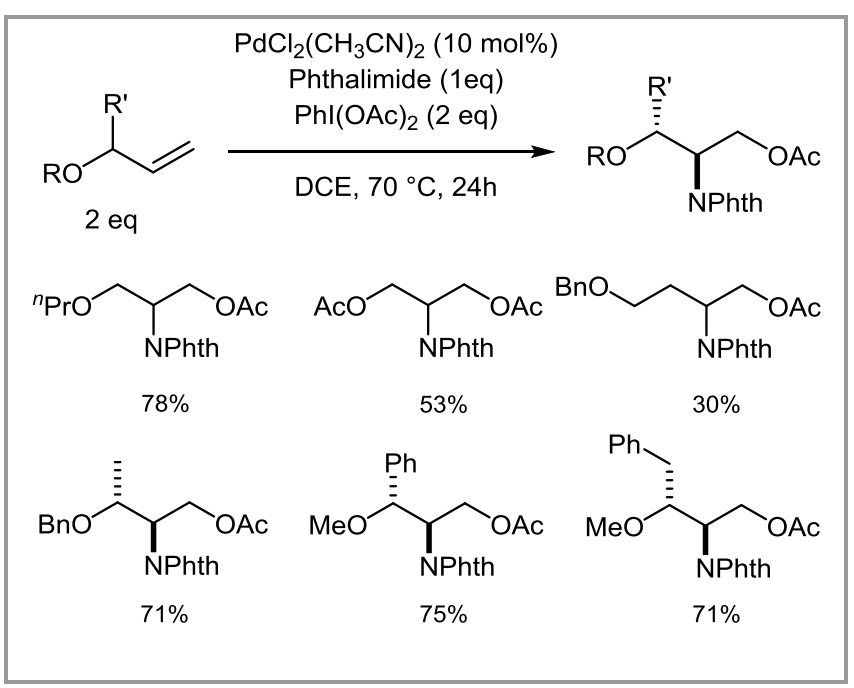

Scheme 95 Aminooxygenation of alkenes using Pd catalysis.

The reaction was proposed to proceed via a classical $\mathrm{Pd}(\mathrm{II}) / \mathrm{Pd}(\mathrm{IV})$ cycle involving three steps: (i) aminopalladation, (ii) oxidation to form a $\mathrm{Pd}(\mathrm{IV}$ ) complex, (iii) reductive elimination from the Pd(IV) complex. To gain a better insight into the reaction mechanism, the authors investigated the behaviour of activated cinnamyl methyl ethers under their conditions (Scheme 96). First of all, to discriminate 
between syn or anti nucleopalladation processes, the reaction was performed without an iodonium salt to allow $\beta$-hydride elimination to take place. Under these conditions, only the $Z$-alkene was isolated from the $\mathrm{cis}$ isomer of the cinnamyl ether while no product was formed from the trans isomer. These results suggested that the aminopalladation may be syn-selective. When the reaction was run in the presence of the iodonium salts, the cis-cinnamyl methyl ether was transformed into the anti product shown by an anti-addition of the phthalimide and acetate across the double bond. As a consequence, the reductive elimination is proposed to occur with an inversion of configuration.

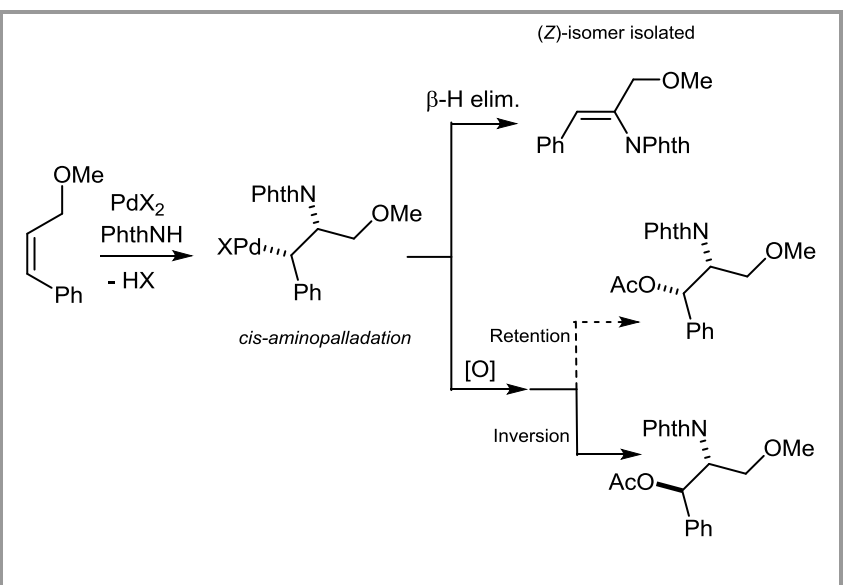

Scheme 96 Aminooxygenation of Z-cinnamyl ethers to determine the most likely pathway for the reaction.

Independently, Sanford et al. have reported an analogous methodology to access substituted tetrahydrofurans via palladium catalysed aminooxygenation of 3-alken-1-ols involving an intermolecular aminopalladation step (Scheme 97). ${ }^{141}$ As previously described, phthalimide was employed as the nitrogen source along with $\mathrm{Pd}(\mathrm{OAc})_{2}$ and an iodonium salt. Importantly, silver tetrafluoroborate was required to improve the conversion. It is interesting to note that intermolecular aminoacetoxylation did not occur under the reaction conditions as the alkoxide group is a better nucleophile than the acetate and can react intramolecularly. Moreover, no by-product of $\beta$ hydride elimination was observed, probably due to the coordination of the alkoxy-moiety, which completes the coordination sphere around the metal centre and slows the $\beta$-hydride elimination process. Finally, 3alken-1-ols with an aromatic allylic substituent afforded 3,4-disubstituted tetrahydrofurans in good yields and modest to high diastereoselectivities in favour of the anti-product.

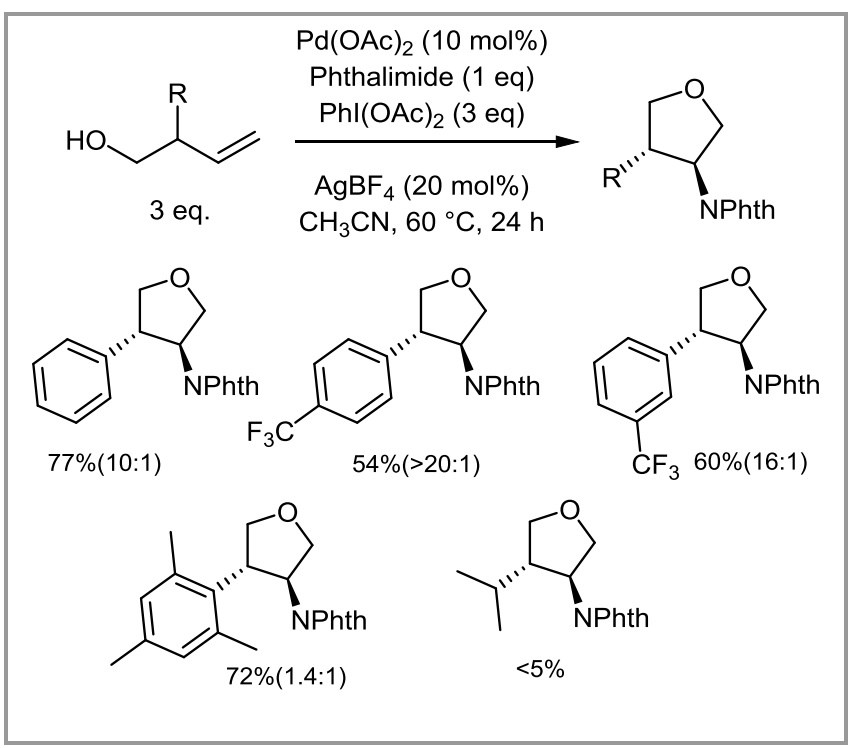

Scheme 97 Aminooxygenation of alkenols.

To account for the selectivity observed in this transformation, the authors carried out a mechanistic study using (Z)-4-phenylprop-3-en-ol (Scheme 98). Applying their optimised conditions to this substrate, the trans-disubstituted tetrahydrofuran was obtained in $60 \%$ yield. Moreover, when the reaction was performed without iodonium salt, the $Z$-product of $\beta$ hydride elimination was obtained as the major compound. In analogy with Stahl's experiments, these results suggested that cis-aminopalladation takes place. In addition, to rationalise the formation of the trans-2,3-disubstituted product the authors suggested that the reductive elimination may happen with retention of configuration in these reactions.

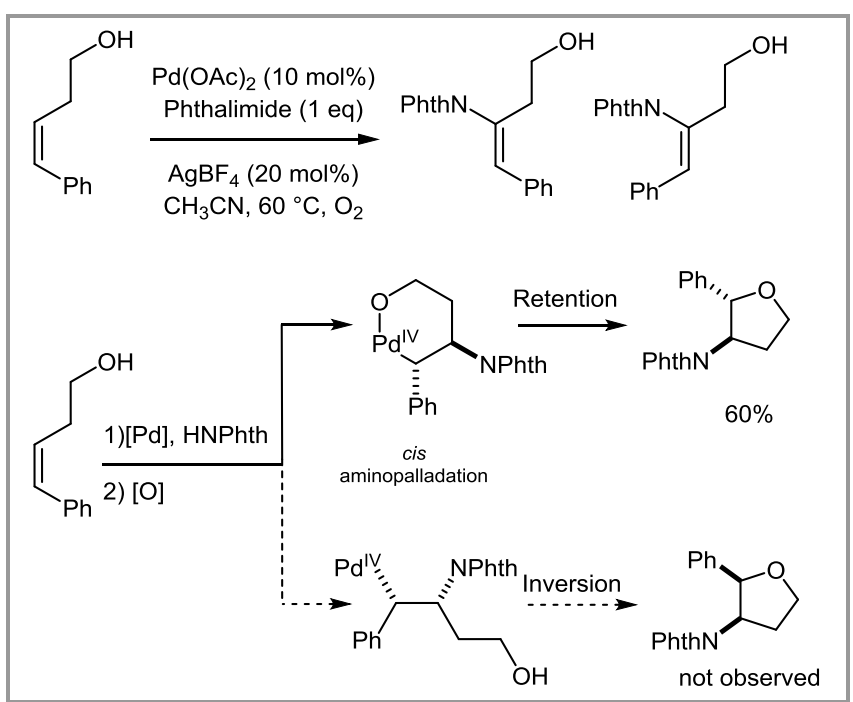

Scheme 98 Experiments to probe the stereochemical pathway of the intramolecular aminooxygenation reactions.

Finally, more recently a collaborative study conducted between Muñiz, Stahl and Liu resulted in a new procedure for the palladium-catalysed intermolecular aminoacetoxylation of alkenes (Scheme 99). ${ }^{142} \mathrm{~A}$ significant improvement in this work was the use of a stoichiometric amount of alkene compared to 
previously reported methods. The optimised set of conditions allowed conversion of terminal but also internal alkenes into difunctionalised products. For internal $Z$-alkenes, the process was diastereoselective with complete formation of the anti-product.

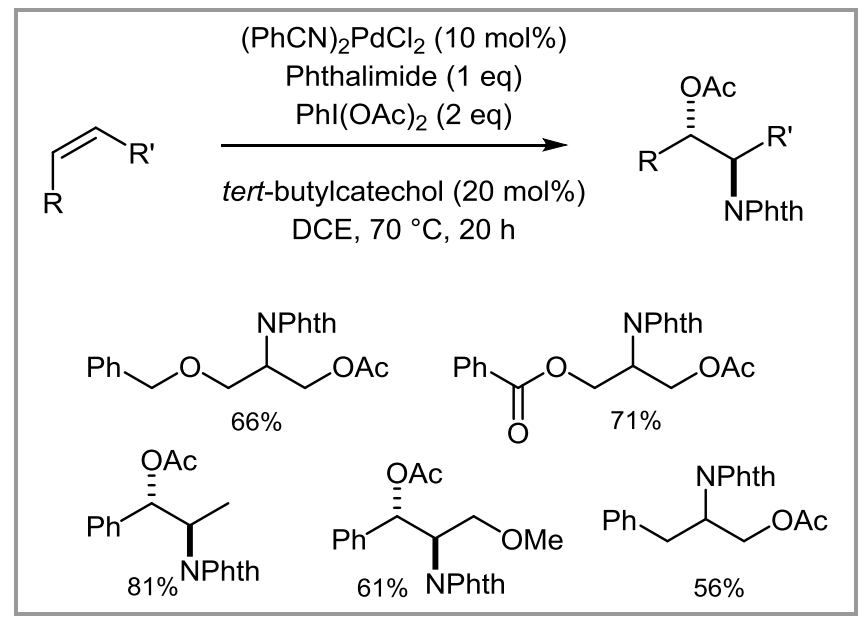

Scheme 99 Intramolecular aminooxygenation of $Z$-alkenes.

In the course of this investigation, the authors highlighted an interesting dependence of the stereoselectivity of the aminopalladation step on the nature of the oxidant employed. Indeed, based on isotopic labeling experiments, they demonstrated that $\mathrm{PhI}(\mathrm{OAc})_{2}$ promoted a trans-aminopalladation in contrast to $\mathrm{O}_{2}$ which induced a syn-selective aminopalladation process.

\subsection{Aminocarbonylation Reactions}

Palladium-catalysed aminocarbonylation consists of an aminopalladation step followed by carbon monoxide insertion into the resulting $\mathrm{Pd}-\mathrm{C}$ bond to form a Pd-acyl intermediate, which can then be intercepted by a nucleophile. The main difficulty in this process is that under a $\mathrm{CO}$ atmosphere, reduction of $\mathrm{Pd}(\mathrm{II})$ can be much faster than nucleopalladation, and this can cause catalyst decomposition. Liu and coworkers have recently developed a methodology which seemed to bypass this decomposition pathway (Scheme 100). ${ }^{143}$ Indeed their strategy relied on the use of an iodonium salt to increase electrophilicity of the palladium centre and accelerate the aminopalladation step. The optimised conditions involved $\mathrm{Pd}(\mathrm{TFA})_{2}(10 \mathrm{~mol} \%)$ with $\mathrm{PhI}\left(\mathrm{O}_{2} \mathrm{CAd}\right)_{2}(2$ eq.) at $60{ }^{\circ} \mathrm{C}$ under 1 atmosphere of $\mathrm{CO}$. Phthalimide or oxazolidin-2-one were employed as nitrogen sources. Interestingly no aminooxygenation products were observed under these reaction conditions. For the substrate scope, styrenes with electron rich, halide or weakly electron-withdrawing groups on the benzene ring were converted in moderate to high yields (a). In contrast, strong electron withdrawing groups were not well tolerated. Less activated aliphatic alkenes were oxidised in moderate yields (b). Finally, subsequent removal of the phthalimide moiety allowed the synthesis of amino-acids in excellent yields.

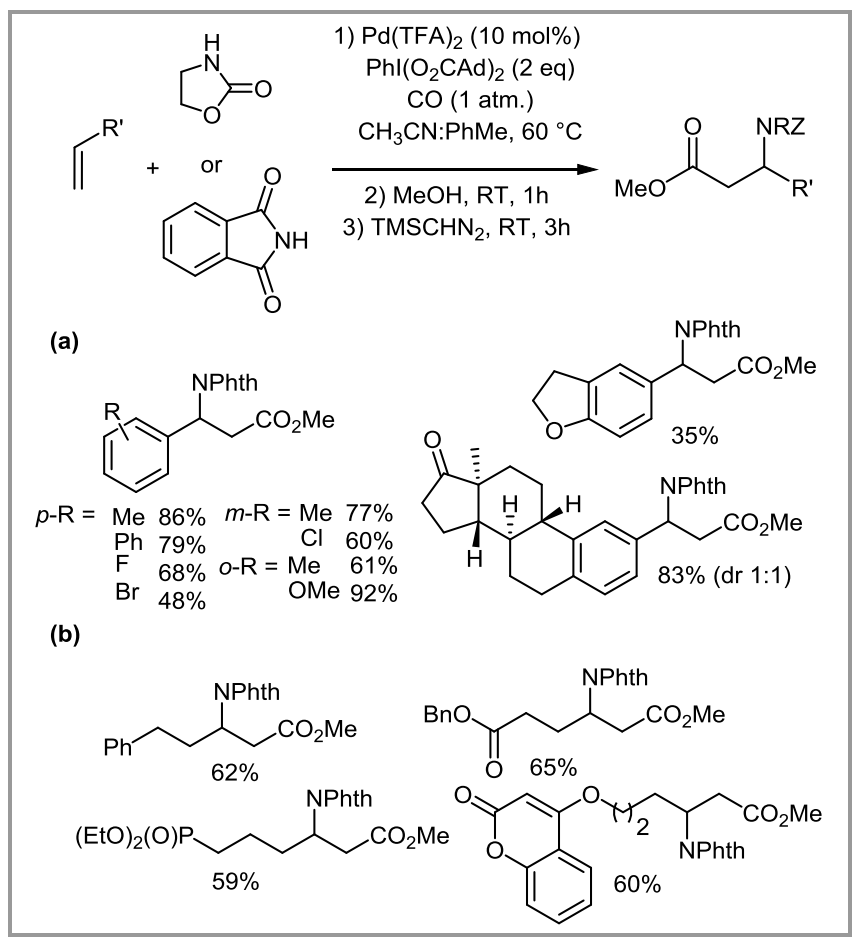

Scheme 100 Aminocarbonylation of terminal alkenes using Pd(II) catalysis.

With support from isotopic labeling experiments, the authors suggested a syn-aminopalladation followed by $\mathrm{CO}$ insertion into the Pd-alkyl bond with retention. The Pd-acyl intermediate is then intercepted by a carboxylate and releases an anhydride which can undergo hydrolysis/esterification. The essential role of the iodonium salt has yet to be determined.

\section{Summary and Conclusions}

Despite the fact that the Wacker oxidation was developed as an industrial process for converting ethylene to acetaldehyde over fifty years ago, the development of more general Pd(II)-mediated alkene oxidation reactions has been relatively slow. Earlier work was largely restricted to the Tsuji-Wacker oxidation of higher terminal alkenes. Sporadic reports over the next few decades included pioneering work on the first examples of allylic oxidation reactions and 1,2-difunctionalisation of alkenes. However, over the past decade or so the field has seen an explosion of interest. Whilst many early processes employed high loadings of palladium catalysts and relied on stoichiometric quantities of copper salts as cooxidants, considerable improvements have been made to the efficiency of the reactions. Low loadings of palladium catalyst are often used as a matter of routine, and simple organic co-oxidants or even molecular oxygen itself can be used to regenerate the palladium catalyst efficiently. A major factor in achieving efficient catalyst turnover is the choice of solvent employed. With simple palladium salts as the catalyst, polar organic solvents (e.g. DMSO, DMA, $\mathrm{MeCN}$ etc) are generally required as these provide efficient ligation of the palladium centre and help to prevent catalyst deactivation and precipitation. This can be problematic, however, as the palladium salts 
are generally less reactive towards alkenes in these polar solvent systems, so higher temperatures are sometimes required. When ligands for the palladium are employed, however, the use of less polar solvents becomes more feasible and this offers the potential for increased reactivity.

A key issue which is still not completely resolved is the elucidation of the factors which determine whether a terminal alkene undergoes oxidation via a Wacker type mechanism (oxypalladation), or through formation of a $\pi$-allyl complex. In general, the latter mechanism is favoured when moderately basic counterions such as acetate are present which can promote the allylic deprotonation. However, even in such systems, Wacker-type oxidation products can still be observed as significant (by)-products, and many of the 1,2-difunctionalisation reactions in section 4 are carried out in the presence of excess acetate. Significant progress has been made in all three areas covered by this review, and there are clear areas of overlap between them where developments in one area could serve to inform the others.

In terms of allylic oxidation reactions, there are now reliable methods available for the direct allylic oxidation of terminal alkenes to give selectively either the linear or branched allylic ester. In terms of the latter transformation, a key goal for future research would be an efficient method for direct asymmetric allylic oxidation, and there are suggestions that this ought to be possible, though it is likely to be far from trivial to achieve. Direct coupling of the allylic oxidation reaction to molecular oxygen as a terminal oxidant is now also possible, and there have been numerous extensions of the classic allylic oxidation protocol to the introduction of other nucleophiles (nitrogen, carbon, fluoride, etc). There remains a significant need to extend this chemistry to less reactive alkene systems such as internal alkenes, trisubstituted alkenes, etc.

Major improvements in the conditions employed for the Wacker oxidation have been made in the past five years. These include a wide range of methods for directly coupling the reaction to molecular oxygen or other low cost oxidants such as peroxides, and the identification of reliable catalyst systems for the oxidation of internal alkenes to ketones. In the latter case, we now have a good understanding of most of the factors that affect the regioselectivity of the reaction with non-symmetrical alkenes. Reaction conditions that promote the regioselective oxidation of terminal alkenes to aldehydes have also been discovered. In many cases, the choice of solvent is extremely important for efficient catalyst activity and turnover. The use of formally cationic palladium

\section{Acknowledgment}

We would like to thank Dr Nick Ray (Argenta) for helpful discussions and for proofreading a draft of this manuscript. LB and TS would like to acknowledge the Engineering and catalysts has been demonstrated to be effective for achieving increased reactivity in many cases. The extension of Wacker type processes to other nucleophiles is still in its infancy, though several methods for direct intermolecular enamine formation with nitrogen nucleophiles have been developed. The development of Pd-mediated methods for direct conversion of alkenes to a range of functionalised derivatives via a Wacker type mechanism offers a potentially useful method for formally achieving 'vinylic C-H activation' and there are considerable opportunities for further developments in this area.

The direct intermolecular 1,2-difunctionalisation of alkenes using $\operatorname{Pd}(\mathrm{II})$ catalysis is still in its infancy, though recent examples building on pioneering work from the 1980s and 1990s have enabled the identification of conditions for a range of oxy- and amino-functionalisation reactions. In general, these methods are restricted in terms of the substrates which can be employed with more reactive compounds (terminal alkenes or internal $Z$-alkenes) being essential. In some cases, the presence of a particular internal 'directing group' is essential for high reactivity. Thus, there are considerable opportunities for further developments in this field by the identification of more active catalyst systems that are able to mediate reactions of less reactive substrates such as internal alkenes or tri-/tetra-substituted double bonds. A major challenge that remains is a full understanding of the factors that control both the nucleopalladation step and the functionalisation of the alkyl-palladium bond. Both syn and anti nucleopalladation processes have been identified, and the functionalisation of the carbon-palladium bond generated can appear to take place either with retention or inversion of configuration. In many cases, this is dependent on the catalyst system and cooxidants employed. The ability to fully predict these functionalisation steps would greatly facilitate the development of Pd-catalysed asymmetric difunctionalisation reactions which might start to provide useful complementary reactions to the well established methods for alkene derivatisation such as the Sharpless asymmetric dihyroxylation and aminohydroxylation reactions.

The palladium(II)-catalysed oxidation of alkenes is a thriving research area that has grown considerably in recent years. It is clear from the above summary that key developments made in any one of the three areas covered by this review have the potential to inform research in the others, and we hope this article will serve to stimulate many future developments in this interesting area.

Physical Sciences Research Council (EP/K001183/1) for financial support. 


\section{References}

(1) Smidt, J.; Hafner, W.; Jira, R.; Sieber, R.; Sedlmeier, J.; Sabel, A. Angew. Chem. 1962, 74, 93.

(2) For a very recent review, see: Kočovský, P.; Bäckvall, J. -E. Chem. Eur. J. 2015, 21, 36.

(3) Keith, J. A.; Henry, P. M. Angew. Chem. Int. Ed. 2009, 48, 9038.

(4) For examples, see: Trost, B. M.; Metzner, P. J. J. Am. Chem. Soc. 1980, 102, 3572

(5) Mann, S. E.; Aliev, A. E.; Tizzard, G. J.; Sheppard, T. D. Organometallics 2011, 30, 1772.

(6) For reviews of the Tsuji-Trost reaction, see: (a) Trost, B. M. J. Org. Chem. 2004, 69, 5813; (b) Trost, B. M.; Crawley, M. L. Chem. Rev. 2003, 103, 2921; (c) Tsuji, J. Tetrahedron 1986, 42, 4361; (d) Trost B. M. Acc. Chem. Res. 1980, 13, 385.

(7) For reviews of the Sharpless asymmetric epoxidation, see: (a) Johnson, R. A.; Sharpless. K. B. In Catalytic Asymmetric Synthesis; 2nd ed.; Ojima, I. Ed.; WileyCVH: New York, 2000; 231-280; (b) Katsuki, T. In Comprehensive Asymmetric Catalysis; 1st ed., Vol. 2; Jacobsen, E. N.; Pfaltz, A.; Yamamoto, H. Eds.; Springer: New York, 1999, 621-648; (c) Jacobsen, E. N In Comprehensive Organometallic Chemistry II, 1st ed., Vol. 12; Abel, E. W.; Stone, F. G. A.; Wilkinson, G. Eds.; Pergamon Press: New York, 1995, 1097-1135; (d) Johnson, R. A.; Sharpless. K. B. In Comprehensive Organic Synthesis, 1st ed., Vol. 7; Trost, B. M.; Fleming, I. Eds.; Pergamon Press: New York, 1991, 389-436.

(8) (a) Nakamura, A.; Nakada, M. Synthesis 2013, 45, 1421; (b) Andrus, M. B.; Lashley, J. C.; Tetrahedron 2002, 58, 845; (c) Eames, J.; Watkinson, M. Angew. Chem. Int. Ed. 2001, 40, 3567; (d) Bulman, P. C.; McCarthy, T. J. In Comprehensive Organic Synthesis; Vol. 7; Trost, B. M.; Fleming, I.; Paquette, L. A. Eds.; Pergamon Press: Oxford, 1991, 83-117; (e) Rabjohn, N. Org. React. 1976, 24, 261; (f) Kharasch, M. S.; Sosnovsky, G.; Yang, N. C. J. Am. Chem. Soc. 1959, 81, 5819; (g) Kharasch, M. S.; Sosnovsky, G. J. Am. Chem. Soc. 1958, 80, 756 .

(9) (a) Moiseev, I. I.; Belov, A. P.; Syrkin, J. K. Izv. Akad. Nauk SSSR, Ser. Khim. 1963, 1527; Bull. Acad. Sci. USSR, Div. Chem. Sci. 1963, 12, 1395; (b) Vargaftik, M. N.; Moiseev, I. I.; Syrkin, J. K.; Yakshin, V. V. Izv. Akad. Nauk SSSR, Ser. Khim. 1962, 930; Bull. Acad. Sci. USSR, Div. Chem. Sci. 1962, 11, 868; (c) Moiseev, I. I.; Vargaftik, M. N.; Syrkin, J. K. Dokl. Akad. Nauk SSSR 1960, 133, 377; (d) Moiseev, I. I.; Vargaftik, M. N.; Syrkin, J. K. Dokl. Akad. Nauk SSSR 1960, 130, 820.

(10) (a) Kitching, W.; Rappoport, Z.; Winstein, S.; Young, W. G. J. Am. Chem. Soc. 1966, 88, 2054; (b) Anderson, C. B.; Winstein, S. J. Org. Chem. 1963, 28, 605.

(11) Heumann, A.; Åkermark, B. Angew. Chem. Int. Ed. 1984, 23, 453 .

(12) (a) Zanoni, G.; Porta, A.; Meriggi, A.; Franzini, M.; Vidari, G. J. Org. Chem. 2002, 67, 6064; (b) Grennberg, H.; Bäckvall, J. E. Chem. Eur. J. 1998, 4, 1083; (c) Grennberg, H.; Simon, V.; Bäckvall, J. E. J. Chem. Soc. Chem. Commun. 1994, 265; (d) Åkermark, B.; Larsson, E. M.; Oslob, J. D. J. Org. Chem. 1994, 59, 5729; (e) Larock, R. C.; Hightower, T. R. J. Org. Chem. 1993, 58, 5298; (f) Hansson, S.; Heumann, A.; Rein, T.;

Åkermark, B. J. Org. Chem. 1990, 55, 975; (g) Byström, S. E.; Larsson, E. M.; Åkermark, B. J. Org. Chem. 1990, 55, 5674; (h) Åkermark, B.; Hansson, S.; Rein, T.; Vågberg, J.; Heumann, A.; Bäckvall, J. E. J. Organomet.
Chem. 1989, 369, 433; (i) McMurry, J. E.; Kočovský, P. Tetrahedron Lett. 1984, 25, 4187.

(13) Bäckvall, J. E.; Byström, S. E.; Nordberg, R. E. J. Org. Chem. 1984, 49, 4619.

(14) Chen, M. S.; White, M. C. J. Am. Chem. Soc. 2004, 126, 1346.

(15) Chen, M. S.; Prabagaran, N.; Labenz, N. A.; White, M. C. J. Am. Chem. Soc. 2005, 127, 6970.

(16) (a) Ammann, S. E.; Rice, G. T.; White, M. C. J. Am. Chem. Soc. 2014, 136, 10834; (b) Osberger, T. J.; White, M. C. J. Am. Chem. Soc. 2014, 136, 11176; (c) Gormisky, P. E.; White, M. C. J. Am. Chem. Soc. 2011, 133, 12584; (d) Stang, E. M.; White, M. C. Angew. Chem. Int. Ed. 2011, 50, 2094; (e) Vermeulen, N. A.; Delcamp, J. H.; White, M. C. J. Am. Chem. Soc. 2010, 132, 11323; (f) Stang, E. M.; White, M. C. Nature Chem. 2009, 1, 547; (g) Covell, D. J.; White, M. C., Angew. Chem. Int. Ed. 2008, 47, 6448; (h) Reed, S. A.; White, M. C. J. Am. Chem. Soc. 2008, 130, 3316; (i) Delcamp, J. H.; White, M. C. J. Am. Chem. Soc. 2006, 128, 15076; (j) Covell, D. J.; Vermeulen, N. A.; Labenz, N. A.; White, M. C. Angew. Chem. Int. Ed. 2006, 45, 8217; (k) Fraunhoffer, K. J.; Prabagaran, N.; Sirois, L. E.; White, M. C. J. Am. Chem. Soc. 2006, 128, 9032; (1) Fraunhoffer, K. J.; Bachovchin, D. A.; White, M. C. Org. Lett. 2005, 7, 223.

(17) (a) Guzman-Perez, A.; Corey, E. J. Tetrahedron Lett. 1997, 38, 5941; (b) Corey, E. J.; Guzman-Perez, A.; Noe, M. C. J. Am. Chem. Soc. 1995, 117, 10805

(18) Messenger, B. T.; Davidson, B. S. Tetrahedron Lett. 2001, 42, 801.

(19) Bartlett, P. A.; Ting, P. C. J. Org. Chem. 1986, 51, 2230.

(20) Saito, T.; Fuwa, H.; Sasaki, M. Org. Lett. 2009, 11, 5274.

(21) Burckhardt, U.; Baumann, M.; Togni, A. Tetrahedron Asymmetry 1997, 8, 155.

(22) Malik, M.; Witkowski, G.; Jarosz, S. Org. Lett. 2014, 16, 3816 .

(23) Ayyagari, N.; Belani, J. D. Synlett 2014, 2350.

(24) Henderson, W. H.; Check, C. T.; Proust, N.; Stambuli, J. P. Org. Lett. 2010, 12, 824.

(25) Le, C.; Kunchithapatham, K.; Henderson, W. H.; Check, C. T.; Stambuli, J. P. Chem. Eur. J. 2013, 19, 11153.

(26) Campbell, A. N.; White, P. B.; Guzei, I. A.; Stahl, S. S. J. Am. Chem. Soc. 2010, 132, 15116.

(27) Mitsudome, T.; Umetani, T.; Nosaka, N.; Mori, K.; Mizugaki, T.; Ebitani, K.; Kaneda, K. Angew. Chem. Int. Ed. 2006, 45, 481 .

(28) Kondo, H.; Yu, F.; Yamaguchi, J.; Liu, G.; Itami, K. Org. Lett. 2014, 16, 4212.

(29) Sharma, A.; Hartwig, J. F. J. Am. Chem. Soc. 2013, 135, 17983.

(30) For a review, see: Mkhalid, I. A. I.; Barnard, J. H.; Marder, T. B.; Murphy, J. M.; Hartwig, J. F. Chem. Rev. 2010, 110, 890 .

(31) (a) Ball, M.; Gaunt, M. J.; Hook, D. F.; Jessiman, A. S.; Kawahara, S.; Orsini, P.; Scolaro, A.; Talbot, A. C.; Tanner, H. R.; Yamanoi, S.; Ley, S. V. Angew. Chem. Int. Ed. 2005, 44, 5433. (b) Gaunt, M. J.; Jessiman, A. S.; Orsini, P.; Tanner, H. R.; Hook, D. F.; Ley, S. V. Org. Lett. 2003, 5, 4819. (c) Gaunt, M. J.; Hook, D. F.; Tanner, H. R.; Ley, S. V. Org. Lett. 2003, 5, 4815.

(32) Check, C. T.; Henderson, W. H.; Wray, B. C.; Vanden Eynden, M. J.; Stambuli, J. P. J. Am. Chem. Soc. 2011, $133,18503$.

(33) Chen, H.; Jiang, H.; Cai, C.; Dong, J.; Fu, W. Org. Lett. 2011, 13, 992 .

(34) Amino Group Chemistry: From Synthesis to the Life Sciences; Ricci, A., Ed.; Wiley-VCH: Weinheim, 2008. 
(35) For selected reviews, see: (a) Breder, A. Synlett 2014 899; (b) McDonald, R. I.; Liu, G.; Stahl, S. S. Chem. Rev. 2011, 111, 2981; (c) Zalatan, D. N.; DuBois, J. Top. Curr. Chem. 2010, 292, 347; (d) Davies, H. M. L.; Manning, J. R. Nature 2008, 451, 417; (e) Davies, H. M. L.; Long, M. S. Angew. Chem. Int. Ed. 2005, 44, 3518.

(36) Fraunhoffer, K. J.; White, M. C. J. Am. Chem. Soc. 2007, 129, 7274.

(37) Taylor, L. D.; Pluhar, M.; Rubin, L. E. J. Polym. Sci. B Polym. Lett. 1967, 5, 77.

(38) Trost, B. M.; Sudhakar, A. R. J. Am. Chem. Soc. 1987, 109, 3792.

(39) Delcamp, J. H.; Brucks, A. P.; White, M. C. J. Am. Chem. Soc. 2008, 130, 11270.

(40) Jiang, C.; Covell, D. J.; Stepan, A. F.; Plummer, M. S.; White, M. C. Org. Lett. 2012, 14, 1386.

(41) Wu, L.; Qiu, S.; Liu, G. Org. Lett. 2009, 11, 2707.

(42) Reed, S. A.; White, M. C. J. Am. Chem. Soc. 2008, 130, 3316.

(43) Reed, S. A.; Mazzotti, A. R.; White, M. C. J. Am. Chem. Soc. 2009, 131, 11701.

(44) Shimizu, Y.; Obora, Y.; Ishii, Y. Org. Lett. 2010, 12, 1372.

(45) Yin, G.; Wu, Y.; Liu, G. J. Am. Chem. Soc. 2010, 132, 11978.

(46) Qi, X.; Rice, G. T.; Lall, M. S.; Plummer, M. S.; White, M. C. Tetrahedron 2010, 66, 4816.

(47) Young, A. J.; White, M. C. J. Am. Chem. Soc. 2008, 130, 14090.

(48) Young, A. J.; White, M. C. Angew. Chem. Int. Ed. 2011, $50,6824$.

(49) Trost, B. M.; Donckele, E. J.; Thaisrivongs, D. A.; Osipov, M.; Masters, J. T. J. Am. Chem. Soc. 2015, 137, 2776.

(50) Jiang, H.; Yang, W.; Chen, H.; Li, J.; Wu, W. Chem. Commun. 2014, 50, 7202.

(51) Wang, G.-W.; Zhou, A.-X.; Li, S.-X.; Yang, S.-D. Org. Lett. 2014, 16, 3118.

(52) Chen, H.; Cai, C.; Liu, X.; Li, X.; Jiang, H. Chem. Commun. 2011, 47, 12224.

(53) For a review, see: Purser, S.; Moore, P. R.; Swallow, S.; Gouverneur, V. Chem. Soc. Rev. 2008, 37, 320.

(54) Braun, M.-G.; Doyle, A. G. J. Am. Chem. Soc. 2013, $135,12990$.

(55) Deng, H.-P.; Eriksson, L.; Szabó, K. J. Chem. Commun. 2014, 50, 9207.

(56) (a) Kirai, N.; Iguchi, S.; Ito, T.; Takaya, J.; Iwasawa, N. Bull. Chem. Soc. Jpn. 2013, 86, 784. (b) Selander, N.; Willy, B.; Szabó, K. J. Angew. Chem. Int. Ed. 2010, 49, 4051. (c) Olsson, V. J.; Szabó, K. J. J. Org. Chem. 2009 74, 7715. (d) Olsson, V. J.; Szabó, K. J. Org. Lett. 2008, $10,3129$.

(57) Tao, Z.-L.; Li, X.-H.; Han, Z.-Y.; Gong, L.-Z. J. Am. Chem. Soc. 2015, 137, 4054.

(58) Larsson, J. M.; Zhao, T. S. N.; Szabó, K. J. Org. Lett. 2011, 13, 1888 .

(59) Kovács, G.; Stirling, A.; Lledós, A.; Ujaque, G. Chem. Eur. J. 2012, 18, 5612.

(60) For a typical procedure, see: Tsuji, J.; Nagashima, H.; Nemoto, H. Org. Synth. Coll. 1990, 7, 137.

(61) Muzart, J. Tetrahedron 2007, 63, 7505.

(62) Kobayashi, T.; Kon, Y.; Abe, H.; Ito, H. Org. Lett. 2014, 16, 6397.

(63) Becalli, E. M.; Broggini, G.; Martinelli, M.; Sottocornola, S. Chem. Rev. 2007, 107, 5318.

(64) Cornell, C. N.; Sigman, M. S. J. Am. Chem. Soc. 2005, $127,2796$.
(65) Michel, B. W.; Camelio, A. M.; Cornell, C. N.; Sigman, M. S. J. Am. Chem. Soc. 2009, 131, 6076.

(66) Mimoun, H.; Charpentier, R.; Mitschler, A.; Fischer, J.; Weiss, R. J. Am. Chem. Soc. 1980, 102, 1047.

(67) Fernandes, R. A.; Chaudhari, D. A. J. Org. Chem. 2014, 79, 5787.

(68) Fernandes, R. A.; Bethi, V. Tetrahedron 2014, 70, 4760

(69) a) Cornell, C. N.; Sigman, M. S. Org. Lett. 2006, 8, 4117 ; b) For a relevant review article in this area, see: Gligorich, K. M.; Sigman, M. S. Chem. Commun. 2009, 3854.

(70) Wang, Y. -F.; Gao, Y. -R.; Mao, S.; Zhang, Y. -L.; Guo, D. -D.; Yan, Z. -L.; Guo, S. -H.; Wang, Y. -Q. Org. Lett. 2014, 16, 1610.

(71) Kang, S. -K.; Jung, K. -Y.; Chung, J. -U.; Namkoong, E. -Y.; Kim, T. -H. J. Org. Chem. 1995, 60, 4678.

(72) Dong, J. J.; Browne, W. R.; Feringa, B. L. Angew. Chem. Int. Ed. 2015, 54, 734.

(73) Yamamoto, M.; Nakaoka, S.; Ura, Y.; Kataoka, Y. Chem. Commun. 2012, 48, 1165 .

(74) Feringa, B. L. J. Chem. Soc., Chem. Commun. 1986, 909.

(75) Teo, P.; Wickens, Z. K.; Dong, G.; Grubbs, R. H. Org. Lett. 2012, 14, 3237.

(76) Dong, J. J.; Fañanás-Mastral, M.; Alsters, P. L.; Browne, W. R.; Feringa, B. L. Angew. Chem. Int. Ed. 2013, 52, 5561.

(77) Weiner, B.; Baeza, A.; Jerphagnon, T.; L. Feringa, B. L. J. Am. Chem. Soc. 2009, 131, 9473.

(78) Dong, J. J.; Harvey, E. C.; Fañanás-Mastral, M.; Browne, W. R.; Feringa, B. L, . J. Am. Chem. Soc. 2014, 136, 17302

(79) Andrews, M. A.; Kelly, K. P. J. Am. Chem. Soc. 1981 , 103, 2894.

(80) Wickens, Z. K.; Skakuj, K.; Morandi, B.; Grubbs, R. H. J. Am. Chem. Soc. 2014, 136, 890.

(81) Wickens, Z. K.; Morandi, B.; Grubbs, R. H. Angew. Chem. Int. Ed. 2013, 52, 11257.

(82) Jiang, Y. -Y.; Zhang, Q.; Yu, H. -Z.; Fu, Y. ACS Catal. 2015, $5,1414$.

(83) Mitsudome, T.; Mizumoto, K.; Mizugaki, T.; Jitsukawa, K.; Kaneda, K. Angew. Chem. Int. Ed. 2010, 49, 1238.

(84) Morandi, B.; Wickens, Z. K.; Grubbs, R. H. Angew. Chem. Int. Ed. 2013, 52, 2944.

(85) a) Bäckvall, J. -E.; Awasthi, A. K.; Renko, Z. D. J. Am. Chem. Soc. 1987, 109, 4750; b) Bäckvall, J. -E.; Hopkins, R. B.; Grennberg, H.; Mader, M. M.; Awasthi, A. K. J. Am. Chem. Soc. 1990, 112, 5160.

(86) Morandi, B.; Wickens, Z. K.; Grubbs, R. H. Angew. Chem. Int. Ed. 2013, 52, 9751.

(87) Lerch, M. M.; Morandi, B.; Wickens, Z. K.; Grubbs, R. H. Angew. Chem. Int. Ed. 2014, 53,8654.

(88) DeLuca, R. J.; Edwards, J. L.; Steffens, L. D.; Michel, B. W.; Qiao, X.; Zhu, C.; Cook, S. P.; Sigman, M. S. J. Org. Chem. 2013, 78, 1682.

(89) For relevant examples, see: a) Joosten, A.; Persson, A. K. Å.; Millet, R.; Johnson, M. T.; Bäckvall, J. -E. Chem. Eur. J. 2012, 18, 15151; b) Lu, Z.; Stahl, S. S. Org. Lett. 2012, 14, 1234; c) Redford, J. E.; McDonald, R. I.; Rigsby, M. L.; Wiensch, J. D.; Stahl, S. S. Org. Lett. 2012, 14, 1242; d) Liu, G.; Stahl, S. S. J. Am Chem. Soc. 2007, 129, 6328 .

(90) Ji, X.; Huang, H.; Wu, W.; Li, X.; Jiang, H. J. Org. Chem. 2013, 78, 11155.

(91) Ji, X.; Huang, H.; Wu, W.; Jiang, H. J. Am. Chem. Soc. 2013, 135, 5286.

(92) Ji, X.; Huang, H.; Xiong, W.; Huang, K.; Wu, W.; Jiang, H. J. Org. Chem. 2014, 79, 7005. 
(93) Mizuta, Y.; Yasuda, K.; Obora, Y. J. Org. Chem. 2013, $78,6332$.

(94) Zhao, M. -N.; Lian, X. -L.; Ren, Z. -H.; Wang, Y. -Y.; Guan, Z. -H. RSC Adv. 2014, 4, 62042.

(95) Wu, G.; Wieping, S. Org. Lett. 2013, 15, 5278.

(96) For a review on dihydroxylation, see: Kolb, H. C.; VanNieuwenhze, M. S.; Sharpless, K. B. Chem. Rev. 1994, 94, 2483.

(97) For reviews on Pd-catalysed difunctionalisation of alkenes, see: (a) Muñiz, K.; Martínez, C. Oxidative Functionalisation of Alkenes, in Metal-Catalyzed CrossCoupling Reactions and More; de Meijere, A.; Bräse, S.; Oestreich, M. Ed.; Wiley-VCH: Weinheim, Germany, 2014; 1259-1314; (b) Jacques, B.; Muñiz, K. Palladium Catalysis for Oxidative 1,2-Difunctionalisation of Alkenes, in Catalyzed Carbon-Heteroatom Bond Formation; Yudin, A. K. Ed., Wiley-VCH: Weinheim, Germany. 2010; 119-135; (c) Jensen, K. H.; Sigman, M. S. Org. Biomol. Chem. 2008, 6, 4083.

(98) a) Muñiz, K. Angew. Chem. Int. Ed. 2009, 48, 9412; b) Sehnal, P.; Taylor, R. J. K.; Fairlamb, I. J. S. Chem. Rev. 2010, 110, 824 .

(99) Stangl, H.; Jira, R. Tetrahedron Lett. 1970, 3589.

(100) Bäckvall, J. E.; B. Åkermark; S.O. Ljunggren. J. Am. Chem. Soc. 1979, 101, 2411.

(101) Bäckvall, J. E. Tetrahedron Lett. 1977, 467.

(102) (a) El-Qisairi, A. K.; Qaseer, H. A.; Henry, P. M. J. Organomet. Chem. 2002, 656, 168; (b) El-Qisairi, A.; Henry, P. M. J. Organomet. Chem. 2000, 603, 50; (c) ElQisairi, A.; Hamed, O.; Henry, P. M. J. Org. Chem. 1998, 63, 2790.

(103) El-Qisairi, A. K.; Qaseer, H. A.; Katsigras, G.; Lorenzi, P.; Trivedi, U.; Tracz, S.; Hartman, A.; Miller, J. A.; Henry, P. M. Org. Lett. 2003, 5, 439.

(104) Chevrin, C.; Le Bras, J.; Hénin, F.; Muzart, J. Synthesis 2005, 15, 2615.

(105) Thiery, E.; Chevrin, C.; Le Bras, J.; Harakat, D.; Muzart, J. J. Org. Chem. 2007, 72, 1859.

(106) Wahlen, J.; De Vos, D. E.; Jacobs, P. A. Org. Lett. 2003, 5, 2293.

(107) Schultz, M. J.; Sigman, M. S. J. Am. Chem. Soc. 2006, 128,1460

(108) Zhang, Y.; Sigman, M. S. J. Am. Chem. Soc. 2007, 129, 3076.

(109) For a review on the use of N-based ligand in Pdcatalysed aerobic oxidation, see: Jin, L.; Lei, A. Sci. China. Chem. 2012, 55, 2027.

(110) Jensen, K. H.; Webb, J. D.; Sigman, M. S. J. Am. Chem. Soc. 2010, 17471.

(111) Li, Y.; Song, D.; Dong, V. M. J. Am. Chem. Soc. 2008, 130, 2962.

(112) Wang, A.; Jiang, H.; Chen, H. J. Am. Chem. Soc. 2009, 131,3846

(113) Wang, A.; Jiang, H. J. Org. Chem. 2010, 75, 2321.

(114) Wang, W.; Wang, F.; Shi, M. Organometallics 2010, 29, 928.

(115) For examples of $N$-heterocyclic carbenes ligand used in Wacker oxidation, see: (a) Scarborough, C. C.; Bergant, A.; Sazama, G. T.; Guzei, I. A.; Spencer, L. C.; Stahl, S. S. Tetrahedron 2009, 65, 5084; (b) Cornell, C. N.; Sigman, M. S. J. Am. Chem. Soc. 2005, 127, 2796; (c) Muñiz, K. Adv. Synth. Catal. 2004, 346, 1425.

(116) Park, C. P.; Lee, J. H.; Yoo, K. S.; Jung, K. W. Org. Lett. 2010, 2300.

(117) Park, J. H.; Park, C. Y.; Song, H. S.; Huh, Y. S.; Kim, G. H.; Park, C. P. Org. Lett. 2013, 15, 752.

(118) Neufeldt, S. R.; Sanford, M. S. Org. Lett. 2013, 15, 46.

(119) Wickens, Z. K.; Guzmán, P. E.; Grubbs, R. H. Angew. Chem. Int. Ed. 2015, 54, 236.
(120) James, D. E.; Stille, J. K. J. Am. Chem. Soc. 1976, 98, 1810.

(121) a) Semmelhack, M. F.; Bodurow, C. J. Am. Chem. Soc. 1984, 106, 1496; b) Semmelhack, M. F.; Zhang, N. J. Org. Chem. 1989, 54, 4483.

(122) Wu, T.; Yin, G.; Liu, G. J. Am. Chem. Soc. 2009, 131, 16354.

(123) Qiu, S.; Xu, T.; Zhou, J.; Guo, Y.; Liu, G. J. Am. Chem. Soc. 2010, 132, 2856.

(124) Bäckvall, J. E. J. C. S. Chem. Commun. 1977, 413.

(125) Bäckvall Jan. E. Tetrahedron Lett. 1978, 163.

(126) Antunes, A. M. M.; Marto, S. J. L.; Branco, P. S.; Prabhakar, S.; Lobo, A. M. Chem. Commun. 2001, 8 , 405.

(127) Han, J.; Li, Y.; Zhi, S.; Pan, Y.; Timmons, C.; Li, G. Tetrahedron Lett. 2006, 47, 7225.

(128) Bar, L. J.; Lloyd-jones, G. C.; Booker-Milburn, K. I. J. Am. Chem. Soc. 2005, 127, 7308.

(129) Du, H.; Zhao, B.; Shi, Y. J. Am. Chem. Soc. 2007, 129, 762.

(130) Du, H.; Yuan, W.; Zhao, B.; Shi, Y. J. Am. Chem. Soc. 2007, 129, 11688.

(131) (a) Du, H.; Zhao, B.; Shi, Y. J. Am. Chem. Soc. 2008, 130, 8590; (b) Du, H.; Yuan, W.; Zhao, B.; Shi, Y. J. Am. Chem. Soc. 2007, 129, 7496.

(132) (a) Streuff, J.; Ho, C. H.; Nieger, M.; Muñiz, K. J. Am. Chem. Soc. 2005, 127, 14586; (b) Muñiz, K.; Hövelmann, C. H.; Streuff, J. J. Am. Chem. Soc. 2008, 130, 763.

(133) Muñiz, K. Angew. Chem. Int. Ed. 2009, 48, 9412.

(134) Muñiz, K.; Kirsch, J.; Chavez, P. Adv. Synth. Catal. 2011, 353, 689.

(135) For an exemple where NFSI was used as oxidant, see: Iglesias, A.; Alvarez, R.; de Lera, A. R.; Muñiz, K. Angew. Chem. Int. Ed. 2012, 51, 2225.

(136) Martínez, C.; Muñiz, K. Angew. Chem. Int. Ed. 2012, 2 , 7031.

(137) Bäckvall J. E. Tetrahedron Lett. 1975, 2225.

(138) Bäckvall J. E.; Bjorkman F. E.; Bystrom, S. E. Tetrahedron Lett. 1982, 23, 943.

(139) Alexanian, E. J.; Lee, C.; Sorensen, E. J. J. Am. Chem. Soc. 2005, 127, 7690

(140) Liu, G.; Stahl, S. S. J. Am. Chem. Soc. 2006, 128, 7179.

(141) Desai, L. V; Sanford, M. S. Angew. Chem. Int. Ed. 2007, 46, 5737.

(142) Mart, C.; Wu, Y.; Weinstein, A. B.; Stahl, S. S.; Liu, G.; Muñiz, K. J. Org. Chem. 2013, 78, 6309.

(143) Cheng, J.; Qi, X.; Li, M.; Chen, P.; Liu, G. J. Am. Chem. Soc. 2015, 137, 2480. 
Please place the biographical sketch here, this will be placed on the first (Short Review) or second page (Review) of the article at the galley proof stage. You may also place the photograph(s) of the authors here, alternatively you can send them by regular mail to the editorial office.

Please place the graphical abstract and short title of the article here. The short title will be used as a running header.

\section{Manuscript submission checklist}

- Statement of significance of work.

- Full mailing address, telephone, and fax numbers, and email address of the corresponding author.

- Graphical abstract.

- 5 key words.

- Paper saved as a PDF file.

- Original Word file.

- Original graphics files.

- Biographical sketches of the authors.

- Photographs of the authors, if not included the hardcopies should be sent by regular mail to the Editorial Office after the manuscript is accepted.

Send all materials on this list to the Editor-in-Chief.

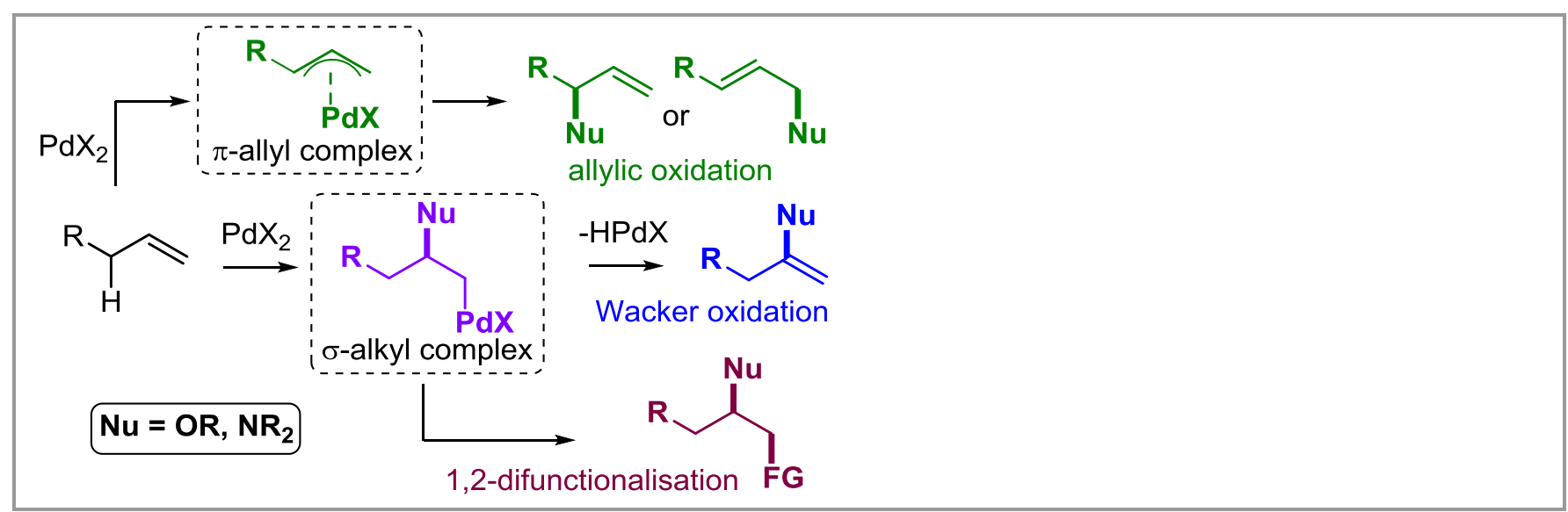

Graphical Abstract 